Supporting Information

\title{
Mechanistic Insights Into the Oxidative Ring Expansion from \\ Penicillin N to Deacetoxycephalosporin C Catalyzed by a Non-heme iron(II) and $\alpha$-KG-Dependent Oxygenase
}

\author{
Yaru Liu ${ }^{1}$, Junyou Shi², Yongjun Liu ${ }^{1, *}$ \\ ${ }^{1}$ School of Chemistry and Chemical Engineering, Shandong University, Jinan, Shandong 250100, China \\ ${ }^{2}$ College of Chemistry and Environmental Science, Qujing Normal University, Qujing, Yunnan 655011, China \\ *Corresponding Author: Tel.: +86 53188365576; fax: +86 53188564464. Email address: \\ yongjunliu_1@sdu.edu.cn
}

Table S1. Relative energies of three reactant structures of Model I at the triplet, quintet and septet states. These structures were selected from the snapshots at 18, 19 and $20 \mathrm{~ns}$ of the MD trajectory. The energy barriers of $\mathrm{H}_{2}$-abstraction (Path_b) at the quintet state are also listed for comparison. All energies are given in $\mathrm{kcal} / \mathrm{mol}$.

\begin{tabular}{ccccc}
\hline & Triplet & Quintet & Septet & Barrier \\
\hline $18 \mathrm{~ns}$ & 12.6 & 0 & 6.5 & 19.5 \\
$19 \mathrm{~ns}$ & 15.1 & 0 & 4.2 & 18.8 \\
$20 \mathrm{~ns}$ & 14.6 & 0 & 6.2 & 18.4 \\
\hline
\end{tabular}

Table S2. Coordinate bond lengths of two reactant models ( $R$ and $\left.R^{\prime}\right)$ at different spin states. The data of $\mathrm{R}^{\prime}$ are listed in parentheses. All distances are given in $\AA$.

\begin{tabular}{ccccc}
\hline & Singlet & Triplet & Quintet & Septet \\
\hline $\mathrm{r}_{\mathrm{Fe}-\mathrm{Osub}}$ & $1.95(1.93)$ & $2.01(1.93)$ & $1.99(2.01)$ & $1.98(1.98)$ \\
$\mathrm{r}_{\mathrm{Fe}-\mathrm{Osucc}}$ & $1.98(1.96)$ & $2.02(1.95)$ & $2.03(2.00)$ & $2.04(2.06)$ \\
$\mathrm{r}_{\mathrm{Fe}-\mathrm{N} 183}$ & $2.12(2.11)$ & $2.08(2.14)$ & $2.12(2.11)$ & $2.23(2.23)$ \\
$\mathrm{r}_{\mathrm{Fe}-\mathrm{O} 185}$ & $2.03(2.05)$ & $2.08(2.05)$ & $2.03(2.10)$ & $2.09(2.07)$ \\
$\mathrm{r}_{\mathrm{Fe}-\mathrm{N} 243}$ & $2.02(2.03)$ & $2.27(2.03)$ & $2.23(2.24)$ & $2.24(2.23)$ \\
\hline
\end{tabular}


Table S3. Calculated spin densities of key atoms and groups at quintet spin state involved in H-abstraction for two models.

\begin{tabular}{ccccccc}
\hline & $\mathrm{Fe}$ & $\mathrm{O}$ & $\mathrm{C} 1$ & $\mathrm{C} 2$ & $\mathrm{~S}$ & $\mathrm{Sub}$ \\
\hline $\mathrm{Ra}$ & 3.27 & 0.47 & 0.01 & 0.00 & 0.00 & 0.10 \\
$\mathrm{TS} 1 \mathrm{a}$ & 4.10 & 0.00 & 0.01 & -0.28 & -0.05 & -0.38 \\
$\mathrm{IM} 1 \mathrm{a}$ & 4.27 & 0.26 & 0.01 & -0.83 & -0.14 & -0.87 \\
$\mathrm{Ra}^{\prime}$ & 3.32 & 0.42 & 0.00 & 0.00 & 0.00 & 0.09 \\
$\mathrm{TS}^{\prime} \mathrm{a}^{\prime}$ & 4.15 & -0.10 & 0.01 & -0.24 & -0.07 & -0.37 \\
$\mathrm{IM} 1 \mathrm{a}^{\prime}$ & 4.28 & 0.23 & 0.01 & -0.81 & -0.17 & -0.86 \\
\hline & $\mathrm{Fe}$ & $\mathrm{O}$ & $\mathrm{C} 1$ & $\mathrm{C} 3$ & $\mathrm{~S}$ & $\mathrm{Sub}$ \\
\hline $\mathrm{TS}^{\prime}$ & 4.08 & 0.03 & -0.01 & -0.28 & -0.04 & -0.39 \\
$\mathrm{IM}^{\prime} 1 \mathrm{~b}$ & 4.24 & 0.26 & -0.01 & -0.85 & -0.07 & -0.81 \\
$\mathrm{TS}^{\prime} \mathrm{b}^{\prime}$ & 4.19 & 0.02 & 0.00 & -0.38 & -0.06 & -0.51 \\
$\mathrm{IM}^{\prime} \mathrm{b}^{\prime}$ & 4.28 & 0.23 & 0.01 & -0.82 & -0.14 & -0.86 \\
\hline
\end{tabular}

Table S4. Calculated spin densities of key atoms and groups at quintet spin state involved in ring cleavage for two models.

\begin{tabular}{ccccccc}
\hline & $\mathrm{Fe}$ & $\mathrm{O}$ & $\mathrm{C} 1$ & $\mathrm{C} 2$ & $\mathrm{~S}$ & $\mathrm{Sub}$ \\
\hline TS2a & 4.27 & 0.25 & 0.07 & -0.62 & -0.41 & -0.87 \\
$\mathrm{IM} 2 \mathrm{a}$ & 4.27 & 0.25 & -0.22 & 0.03 & -0.72 & -0.81 \\
TS2a' & 4.28 & 0.23 & 0.06 & -0.61 & -0.43 & -0.87 \\
$\mathrm{IM} 2 \mathrm{a}^{\prime}$ & 4.27 & 0.23 & -0.08 & -0.09 & -0.79 & -0.86 \\
\hline & $\mathrm{Fe}$ & $\mathrm{O}$ & $\mathrm{C} 1$ & $\mathrm{C} 3$ & $\mathrm{~S}$ & $\mathrm{Sub}$ \\
\hline TS2b & 4.24 & 0.26 & 0.09 & -0.48 & -0.55 & -0.82 \\
IM2b & 4.24 & 0.26 & 0.02 & -0.16 & -0.78 & -0.83 \\
TS2 $\mathrm{b}^{\prime}$ & 4.28 & 0.23 & -0.34 & 0.04 & -0.63 & -0.87 \\
IM2b' & 4.28 & 0.22 & -0.13 & 0.03 & -0.80 & -0.86 \\
\hline
\end{tabular}


Table S5. Calculated spin densities of key atoms and groups at quintet state involved in the ring expansion for two models.

\begin{tabular}{ccccccc}
\hline & $\mathrm{Fe}$ & $\mathrm{O}$ & $\mathrm{C} 1$ & $\mathrm{C} 2$ & $\mathrm{~S}$ & Sub \\
\hline TS3a & 4.27 & 0.25 & -0.26 & 0.04 & -0.69 & -0.86 \\
IM3a & 4.27 & 0.25 & -0.75 & 0.01 & -0.12 & -0.85 \\
TS3a' $^{\prime}$ & 4.27 & 0.23 & -0.34 & 0.04 & -0.63 & -0.87 \\
IM3a' $^{\prime}$ & 4.27 & 0.23 & -0.74 & 0.01 & -0.12 & -0.86 \\
\hline & $\mathrm{Fe}$ & $\mathrm{O}$ & $\mathrm{C} 1$ & $\mathrm{C} 3$ & $\mathrm{~S}$ & $\mathrm{~S}$ \\
\hline TS3b & 4.24 & 0.27 & -0.32 & -0.06 & -0.65 & -0.83 \\
IM3b & 4.26 & 0.23 & -0.72 & 0.00 & -0.11 & -0.82 \\
TS3b' & 4.25 & 0.23 & -0.24 & 0.05 & -0.63 & -0.86 \\
IM3b' $^{\prime}$ & 4.28 & 0.24 & -0.75 & 0.00 & -0.11 & -0.85 \\
\hline
\end{tabular}

Table S6. Calculated spin densities of key atoms and groups at quintet spin states involved in desaturation for two models.

\begin{tabular}{ccccccc}
\hline & $\mathrm{Fe}$ & $\mathrm{O}$ & $\mathrm{C} 1$ & $\mathrm{C} 2$ & $\mathrm{~S}$ & $\mathrm{Sub}$ \\
\hline $\mathrm{TS} 4 \mathrm{a}$ & 4.09 & 0.22 & -0.54 & -0.01 & -0.06 & -0.59 \\
$\mathrm{~Pa}$ & 3.71 & 0.04 & 0.01 & 0.00 & 0.00 & 0.07 \\
$\mathrm{TS} \mathrm{a}^{\prime}$ & 4.07 & 0.18 & -0.48 & 0.00 & -0.06 & -0.53 \\
$\mathrm{~Pa}^{\prime}$ & 3.66 & 0.04 & 0.02 & 0.00 & 0.00 & 0.08 \\
\hline & $\mathrm{Fe}$ & $\mathrm{O}$ & $\mathrm{C} 1$ & $\mathrm{C} 3$ & $\mathrm{~S}$ & $\mathrm{Sub}$ \\
\hline $\mathrm{TS} 4 \mathrm{~b}$ & 4.22 & 0.16 & -0.73 & 0.01 & -0.12 & -0.73 \\
$\mathrm{~Pb}$ & 3.71 & 0.04 & 0.00 & 0.00 & 0.00 & 0.06 \\
$\mathrm{TS} 4 \mathrm{~b}^{\prime}$ & 4.25 & 0.14 & -0.70 & 0.00 & -0.13 & -0.75 \\
$\mathrm{~Pb}^{\prime}$ & 3.66 & 0.04 & 0.02 & 0.00 & 0.01 & 0.00 \\
\hline
\end{tabular}



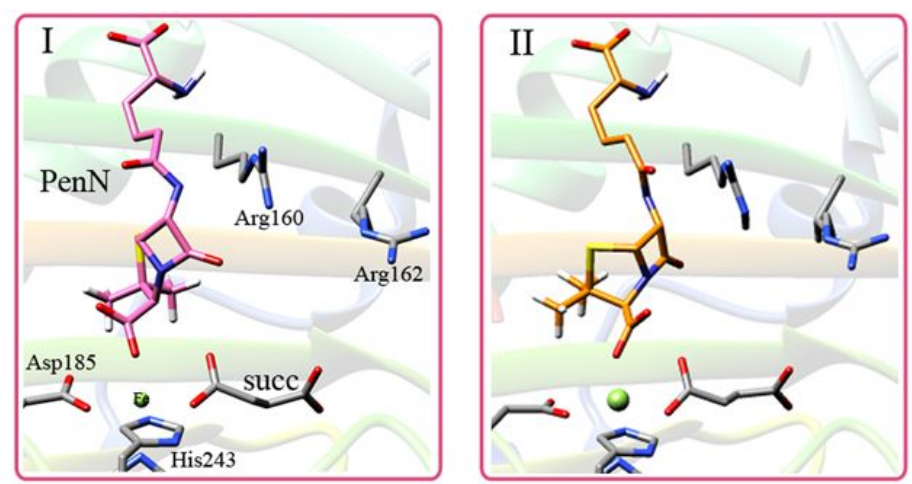

Figure S1. Two representative conformations suitable for reaction obtained from the docking results. Docking details: Before docking, the substrate was first optimized by using the Gaussian 09 package with B3LYP functional and the $6-31 \mathrm{G}(\mathrm{d}, \mathrm{p})$ basis set. The polar hydrogens were added to the protein molecule, and Gasteiger charges were set for all atoms. During the docking, three-dimensional grid map was generated in the active site, which was set to $40 \AA \times 36 \AA \times 40 \AA$ with a grid spacing of $0.375 \AA$. The protein was kept rigid. After 50 independent runs, the docking results were clustered according to a root-mean-square deviation (RMSD) value of $2.0 \AA$.

$\mathrm{a}$

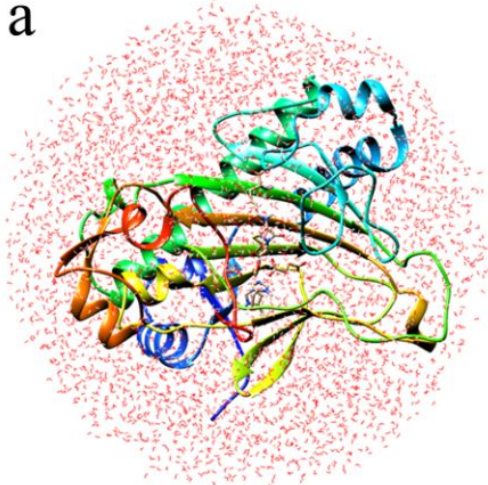

\begin{tabular}{lllllllllll}
\hline Snapshots & $11 \mathrm{~ns}$ & $12 \mathrm{~ns}$ & $13 \mathrm{~ns}$ & $14 \mathrm{~ns}$ & $15 \mathrm{~ns}$ & $16 \mathrm{~ns}$ & $17 \mathrm{~ns}$ & $18 \mathrm{~ns}$ & $19 \mathrm{~ns}$ & $20 \mathrm{~ns}$ \\
\hline $\mathrm{RMSD}(\AA)$ & 0.68 & 0.64 & 0.58 & 0.44 & 0.63 & 0.45 & 0.43 & 0.43 & 0.47 & 0.37 \\
\hline
\end{tabular}

b c
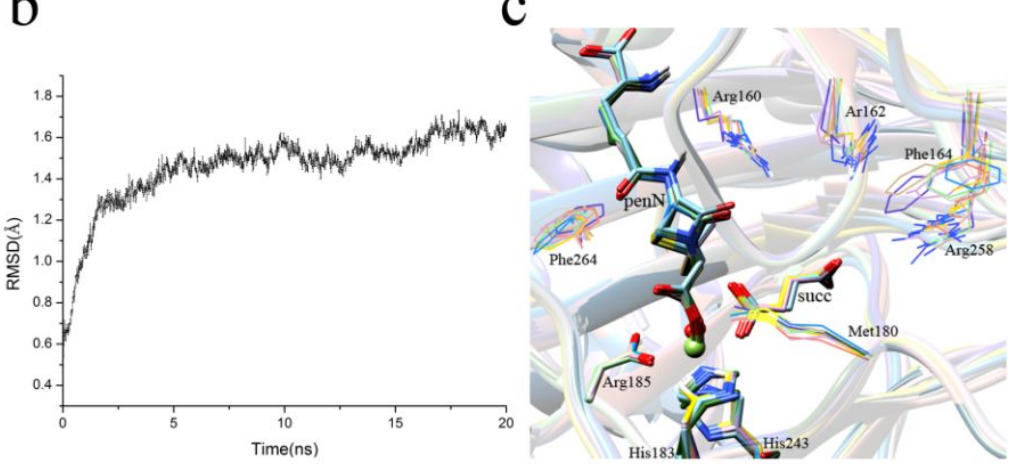

Figure S2. (a) Constructed solvation model for MD simulation using conformation I as the initial structure; (b) RMSD for the backbone atoms of DAOCS enzyme-substrate complex in 20 ns MD simulation, and (c) Superposition of ten QM/MM-optimized structures selected from the MD trajectories at interval of $1 \mathrm{~ns}$ from $11 \mathrm{~ns}$ to $20 \mathrm{~ns}$. The above table shows the RMSD values of backbone atoms of the whole system relative to the average one, which range from 0.37 to $0.68 \AA$. 


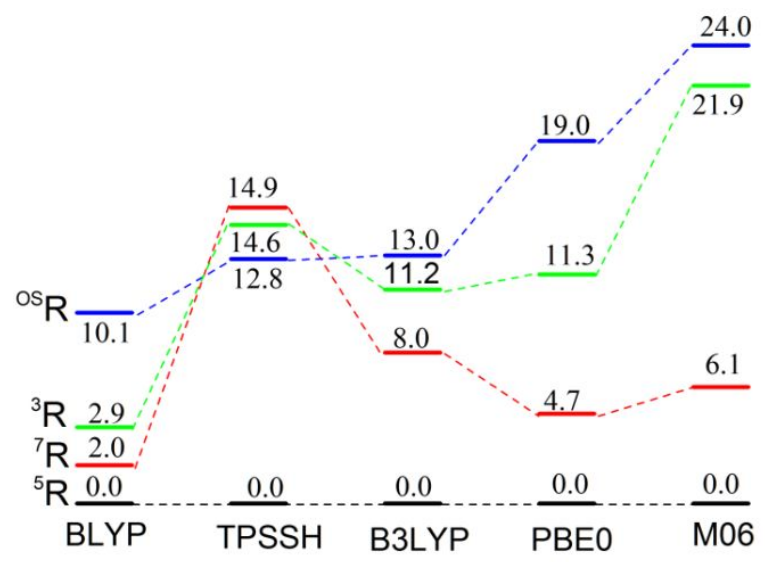

Figure S3. Relative energies (in $\mathrm{kcal} / \mathrm{mol}$ ) for the reactant complex (R) of Model I at the open-shell singlet, triplet, quintet and septet spin states, using different density functionals of BLYP, TPSSH, B3LYP, PBE0 and M06 at B1 level.

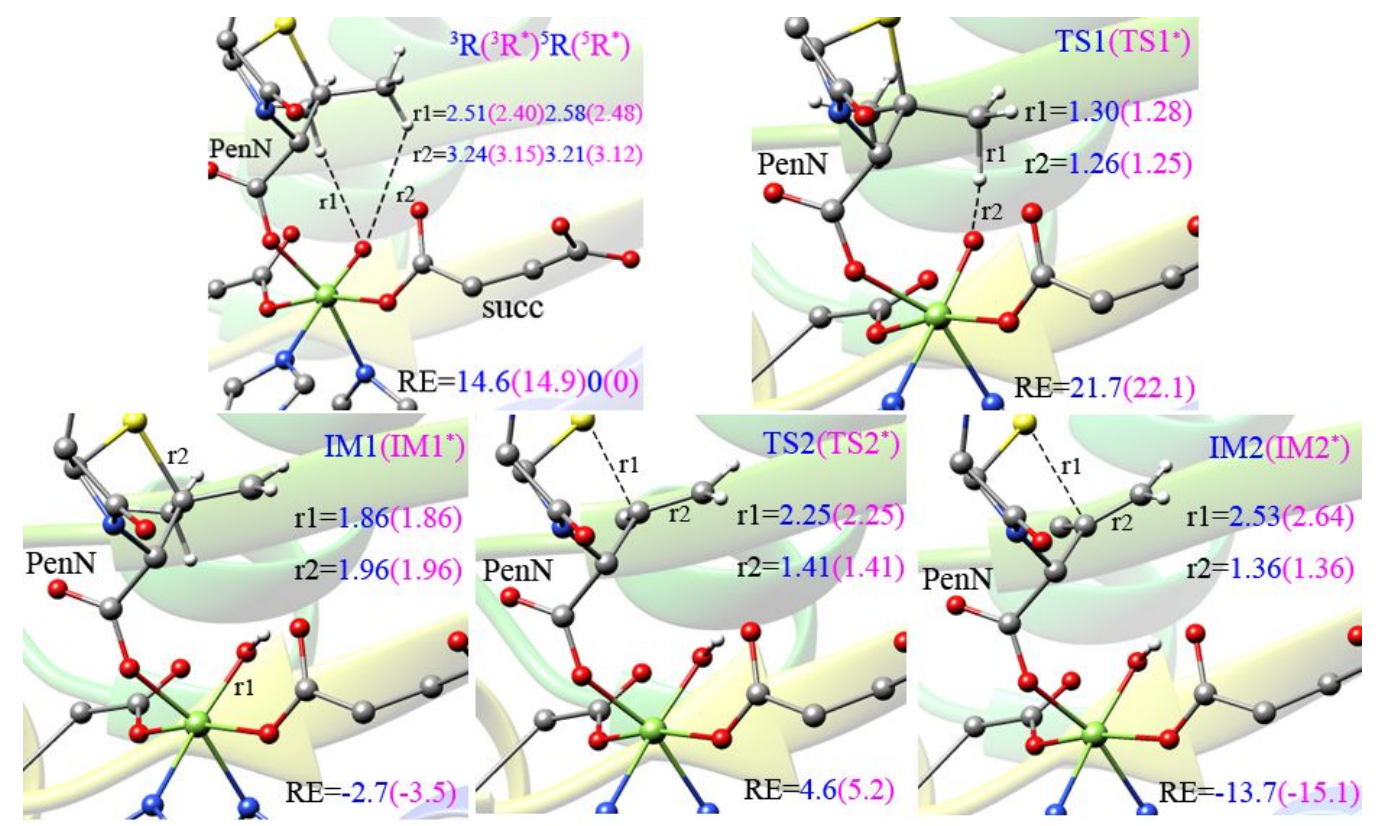

Figure S4. Optimized structures and relative energies (kcal/mol) of some species in Path_a for Model I with B3LYP and with B3LYP-D3 at the quintet state. The results with B3LYP-D3 are marked with asterisk. The energy of reactant $\left({ }^{5} \mathrm{R}\right.$ or $\left.{ }^{5} \mathrm{R}^{*}\right)$ at the quintet state was set to zero. 

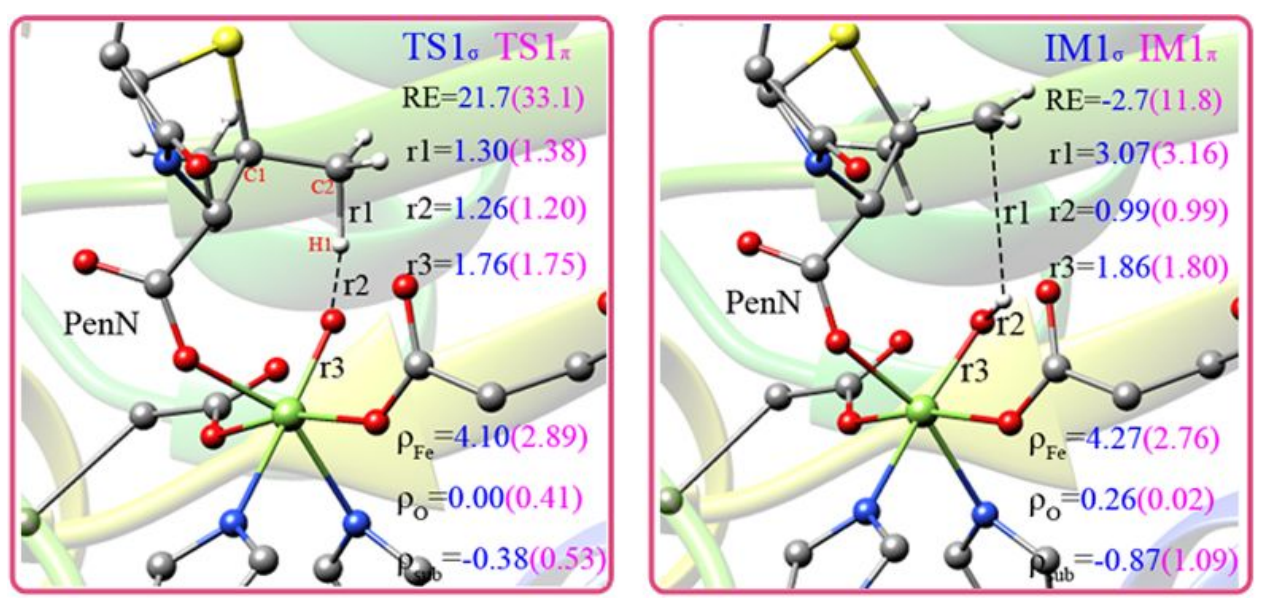

Figure S5. Optimized structures of involved species in $\sigma$ - and $\pi$-channels at the quintet state for Model I in path_a. The data involved in $\pi$-channel are listed in parentheses. All distances are shown in $\AA$. Spin population of key atoms and groups are shown at the bottom, all energies are given in $\mathrm{kcal} / \mathrm{mol}$ relative to ${ }^{5} \mathrm{R}$.

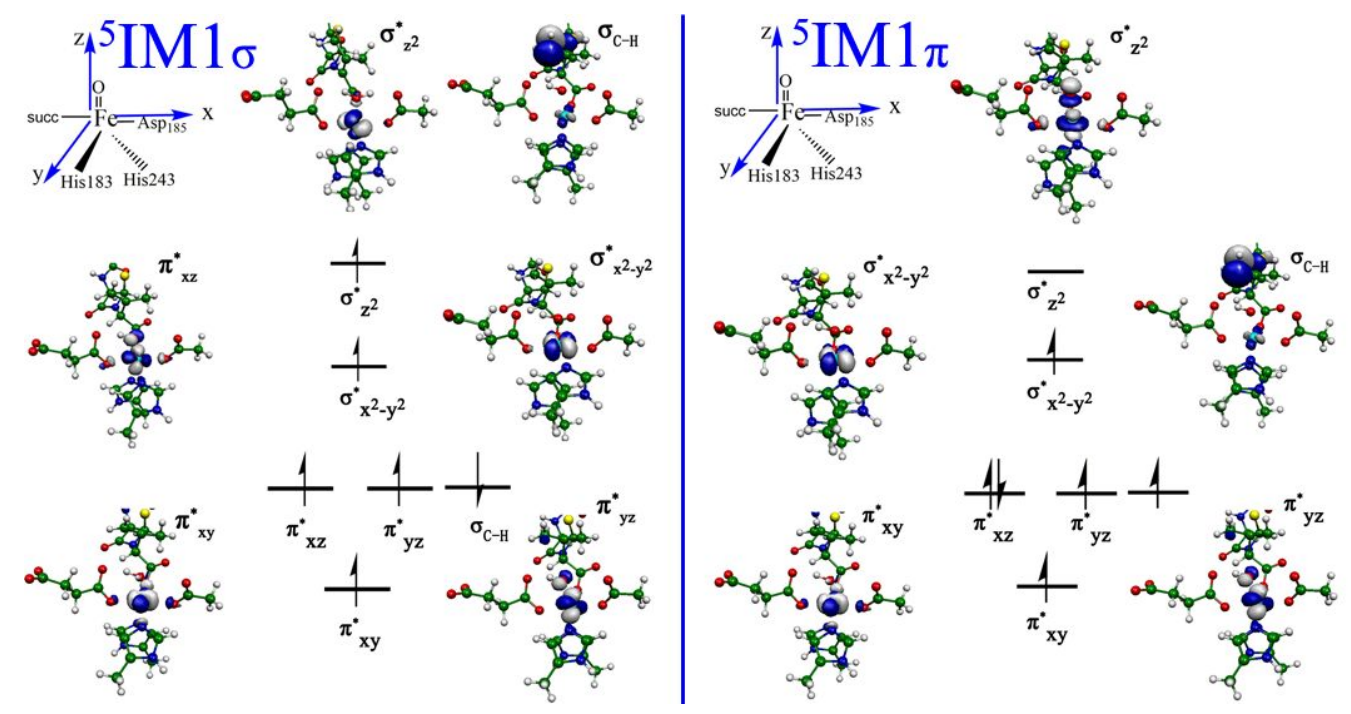

Figure S6. Valent electron orbitals of ${ }^{5} \mathrm{IM} 1 \mathrm{a}$ in $\sigma$ - and $\pi$-channels for model I in Path_a. 

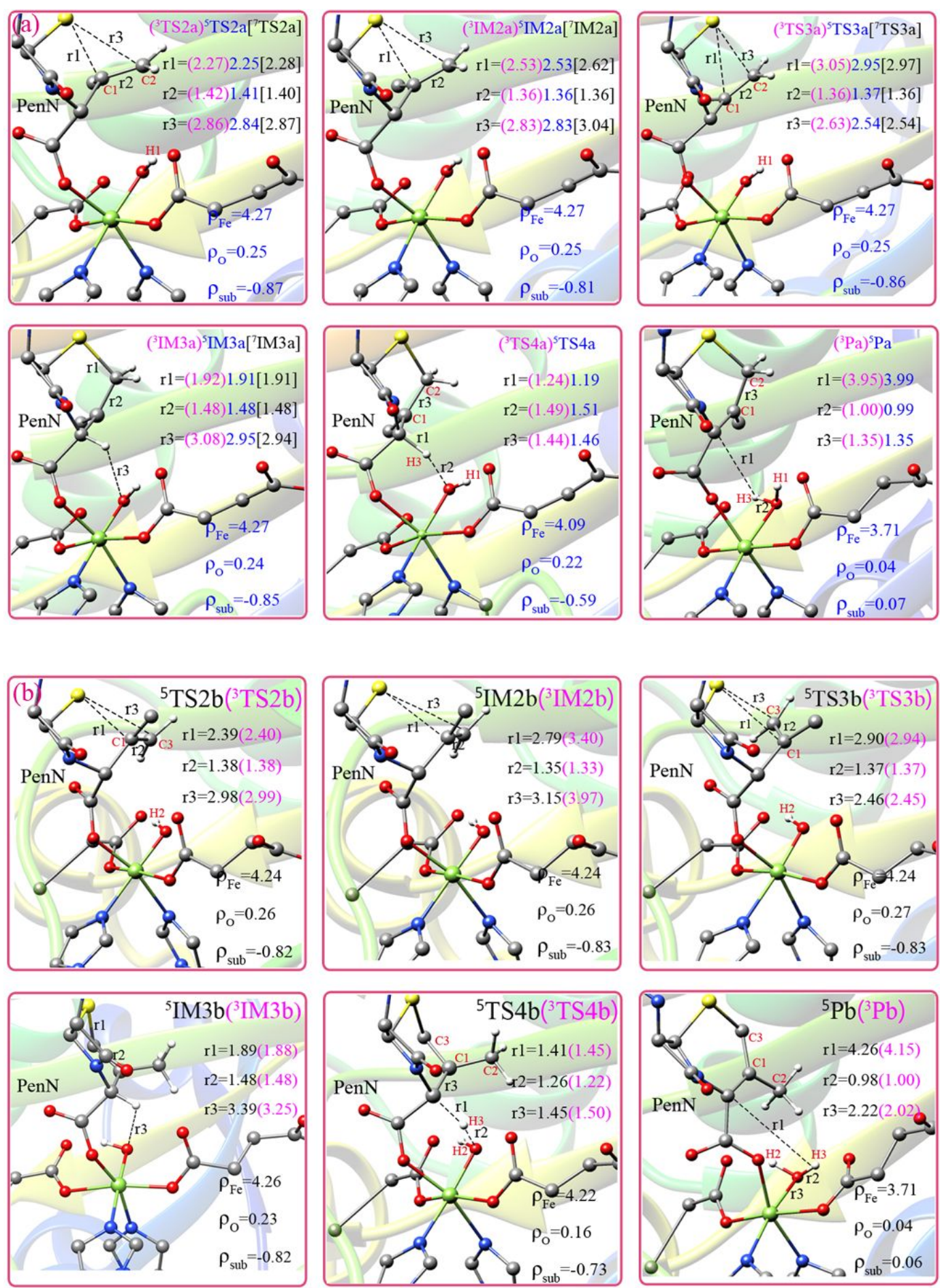

Figure S7. Optimized structures of transition states and intermediates at different spin states for

Model I. (a): Path_a; (b): Path_b. All distances are shown in Å. The spin population of key atoms and groups at the quintet state are shown at the bottom. 


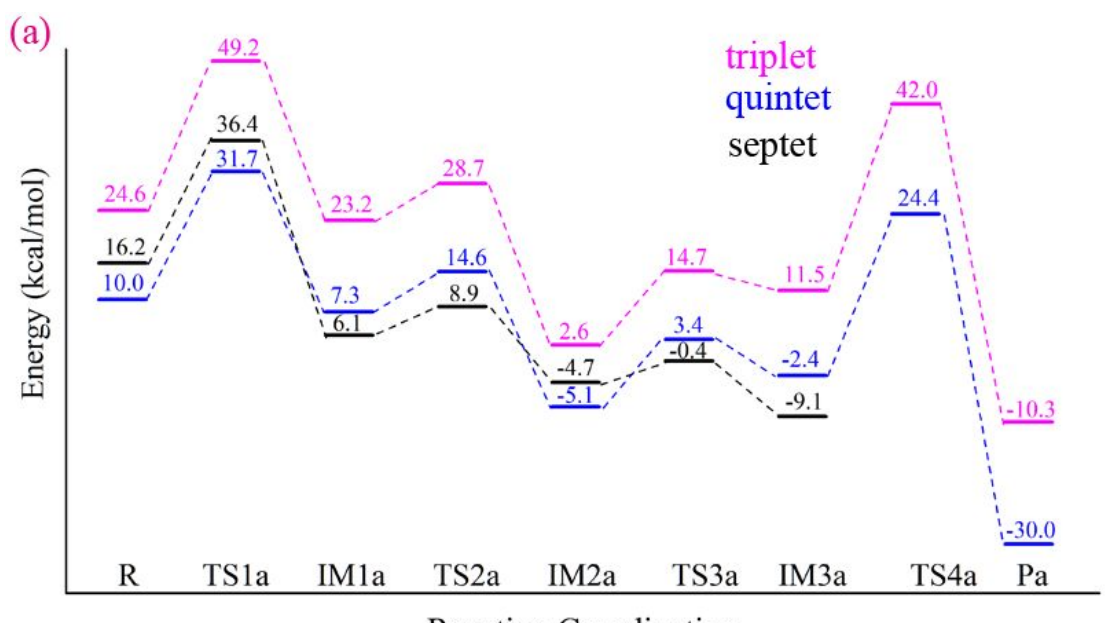

Reaction Coordination

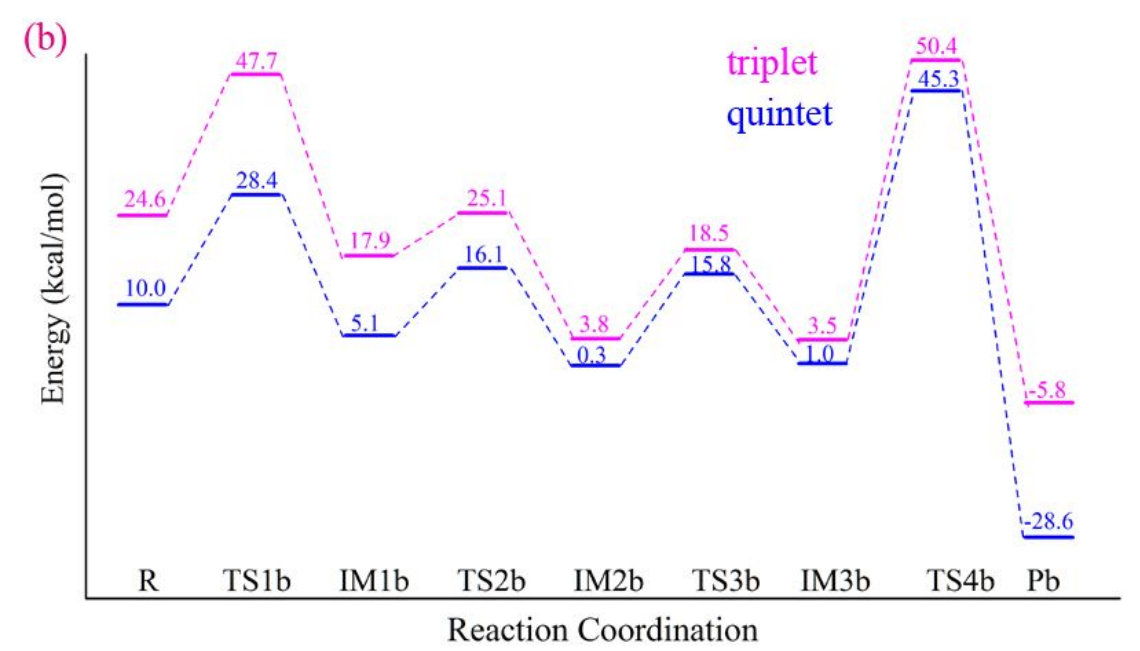

Figure S8. Potential energy profiles with Model I at different spin states in Path_a (a) and Path_b (b). All energies are given in $\mathrm{kcal} / \mathrm{mol}$ relative to ${ }^{5} \mathrm{R}$ '. 

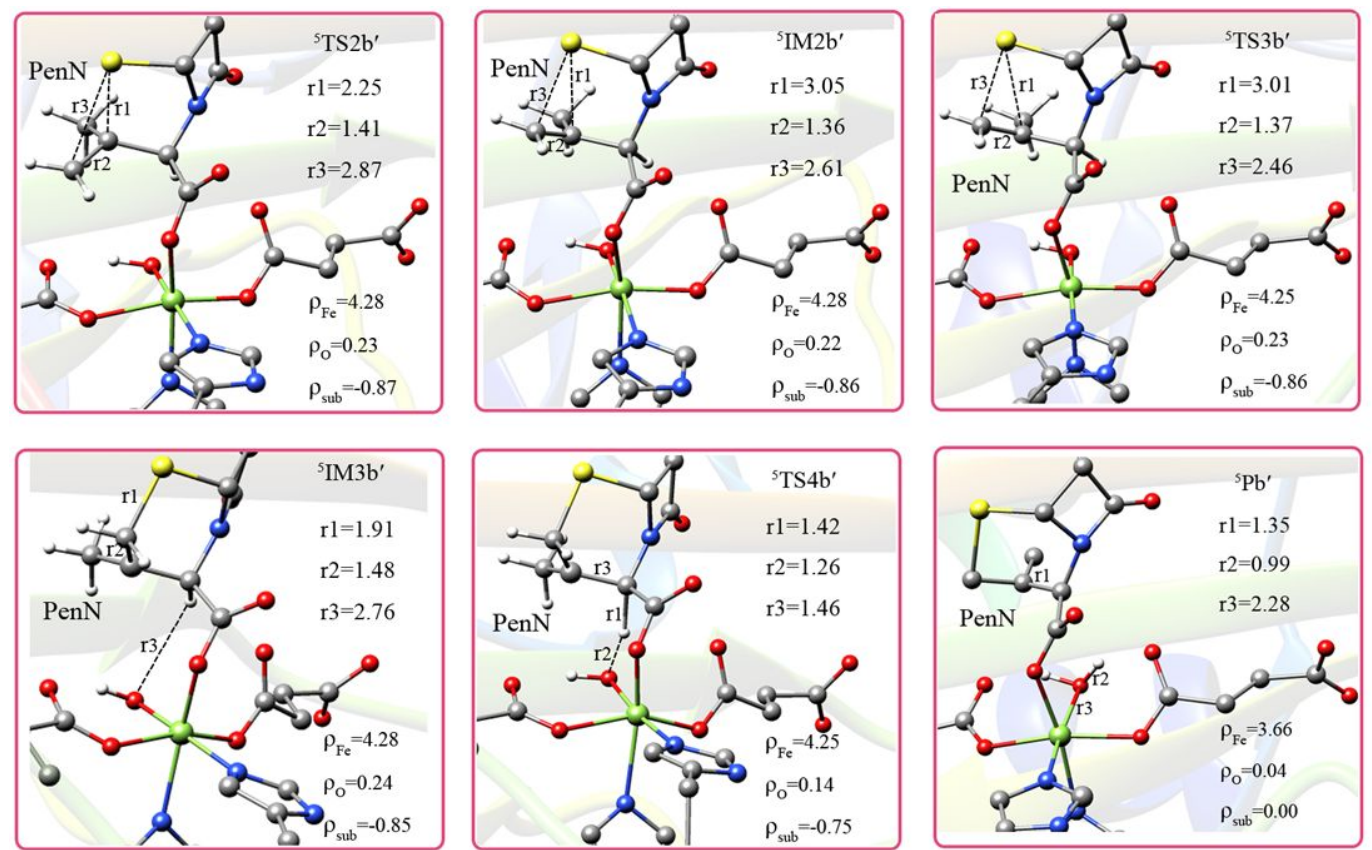

Figure S9. Optimized structures of involved in Path_b at quintet spin state for Model II. All distances are shown in $\AA$. Spin population of key atoms and groups are shown at the bottom.

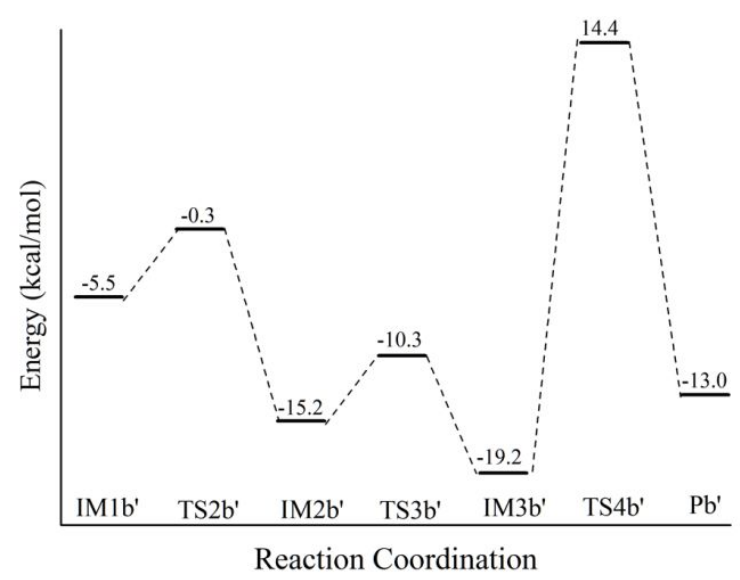

Figure S10. Calculated potential energy profiles with Model II in Path_b at quintet state. All energies are given in $\mathrm{kcal} / \mathrm{mol}$ relative to ${ }^{5} \mathrm{R}$ '. 

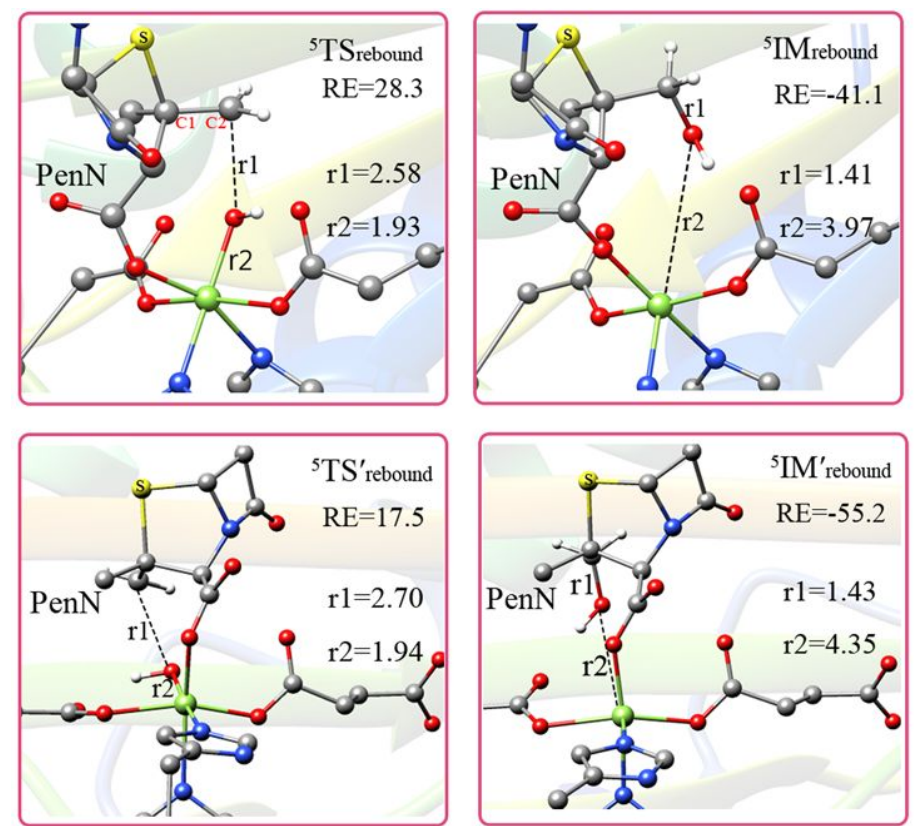

Figure S11. Optimized structures and relative energies for the hydroxylation after the first hydrogen abstraction at quintet state for two models in Path_a. All distances are given in $\AA$ and all energies $(\mathrm{kcal} / \mathrm{mol})$ are given in $\mathrm{kcal} / \mathrm{mol}$ relative to ${ }^{5} \mathrm{R}^{\prime}$.
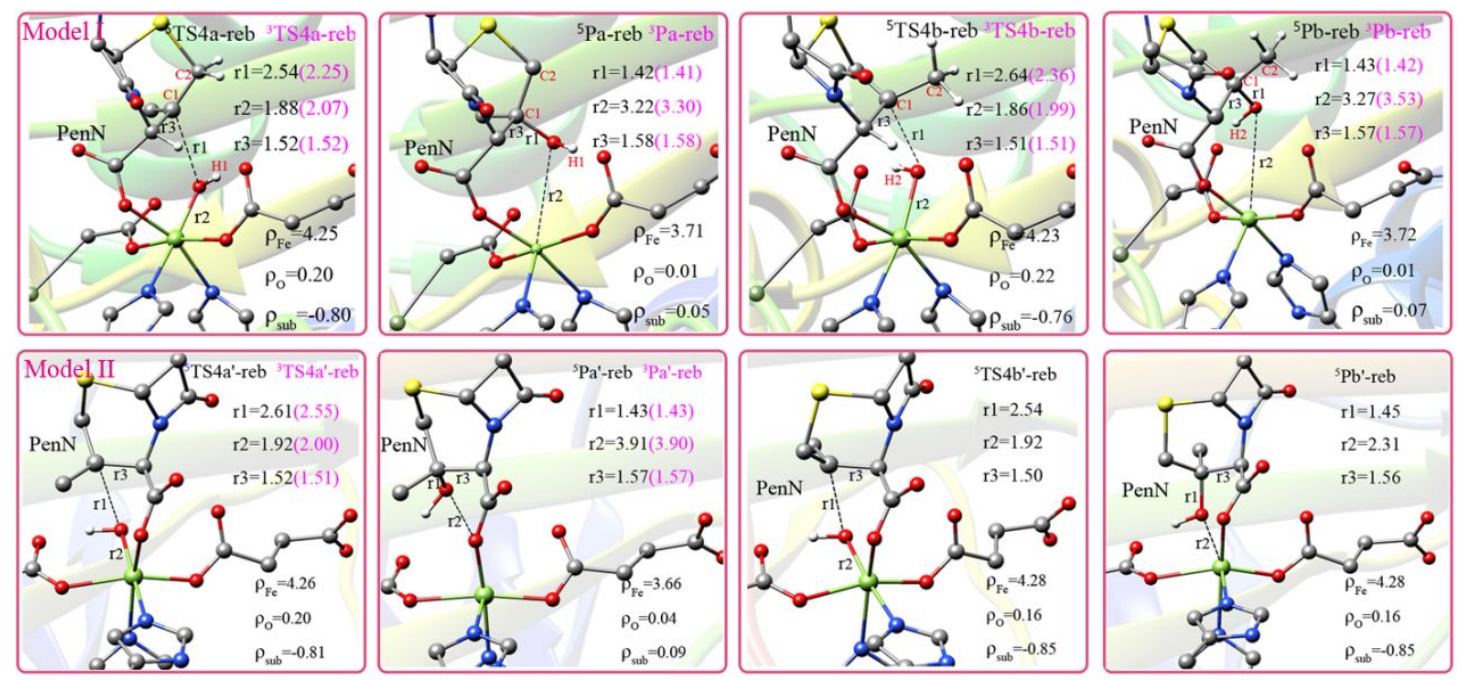

Figure S12. Optimized structures of transition states and the "shunt" products generated by the hydroxylation for two models in both pathways. All distances are given in Å. Spin population of key atoms and groups at the quintet state are shown at the bottom. 


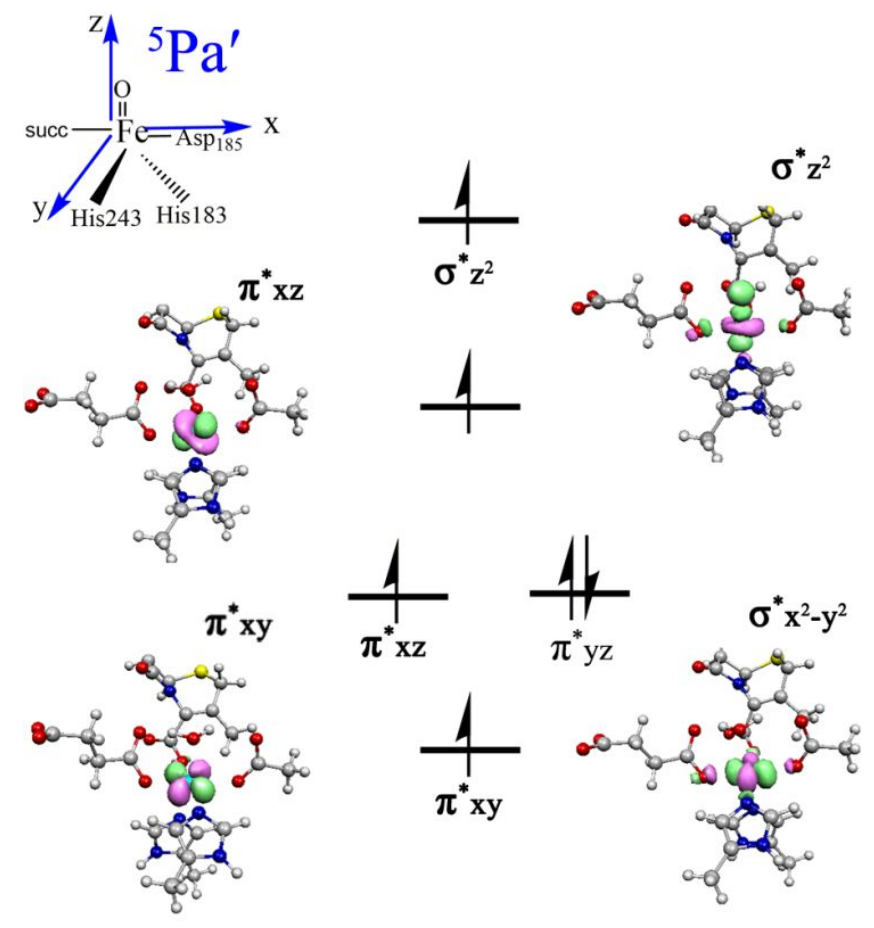

Figure S13. Valent electron orbitals of ${ }^{5} \mathrm{~Pa}^{\prime}$.
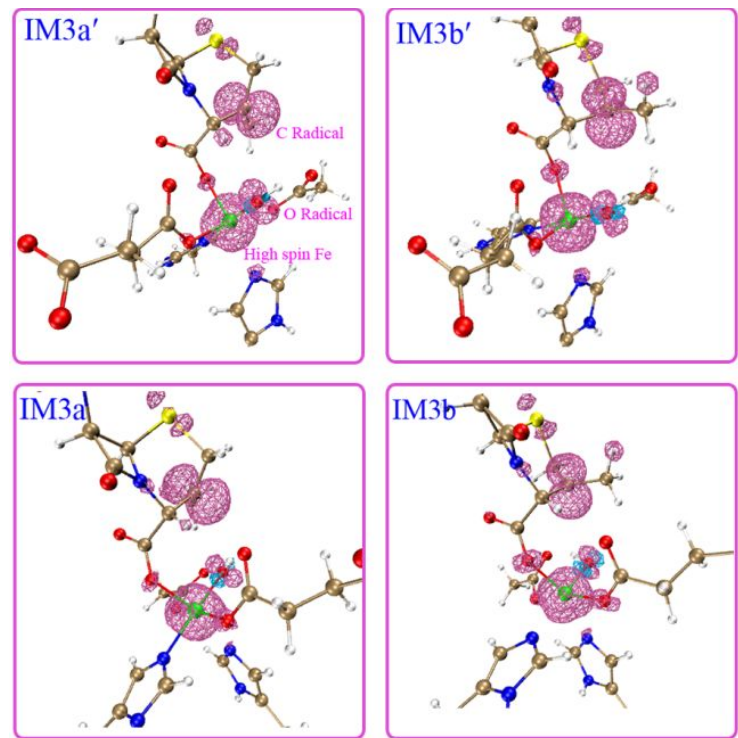

Figure S14. Plots of the total spin surface of four intermediates. 

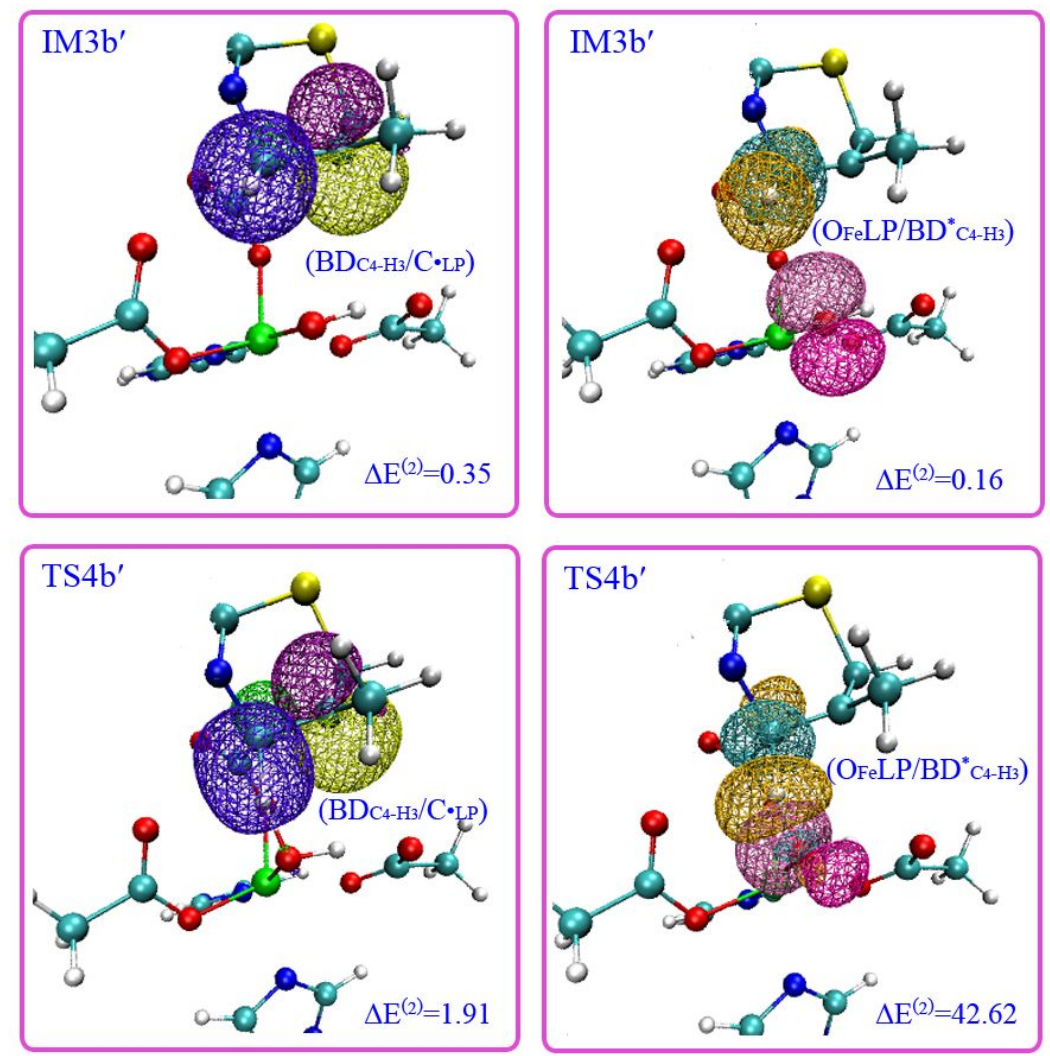

Figure S15. Natural bond orbital (NBO) analysis of ${ }^{5} \mathrm{IM} 3 \mathrm{~b}^{\prime}$ and ${ }^{5} \mathrm{TS} 4 \mathrm{~b}^{\prime}$. LP, lone pair; BD*, antibonding molecular orbital. $\Delta \mathrm{E}^{(2)}$, the second-order perturbative estimations in $\mathrm{kcal} / \mathrm{mol}$.
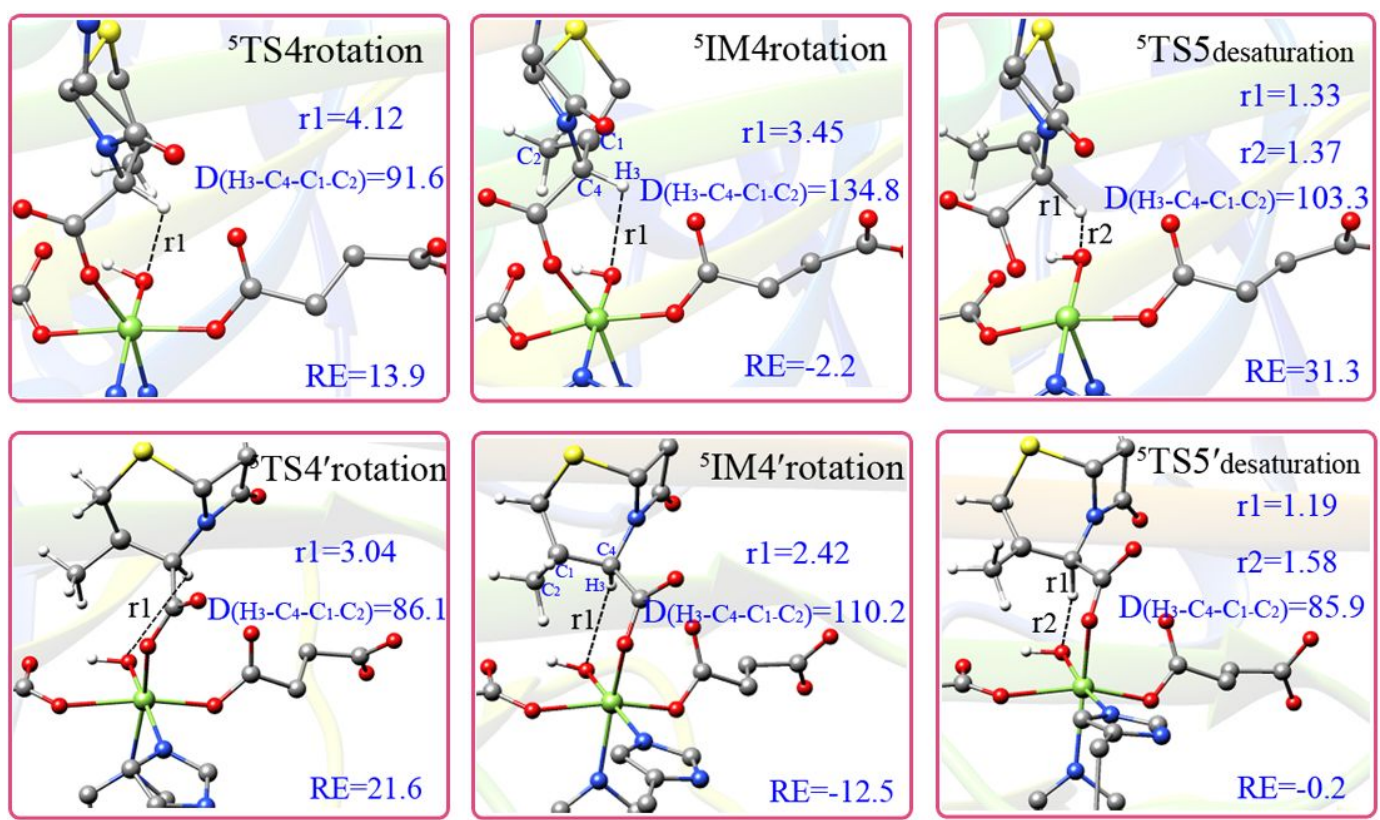

Figure S16. Optimized structures and relative energies for methyl rotation and desaturation for two models in Path_b. All distances are given in angstroms, and angles in degrees. All energies are given in $\mathrm{kcal} / \mathrm{mol}$ relative to ${ }^{5} \mathrm{R}^{\prime}$. 
Coordinates of involved species in Path_a for Model I and Model II at the quintet and triplet states optimized by B3LYP functional at B1 level.

\begin{tabular}{|c|c|c|c|}
\hline \multicolumn{4}{|c|}{ Coordinate of ${ }^{5} \mathrm{R}$} \\
\hline $\mathrm{C}$ & 0.174 & 1.447 & 10.742 \\
\hline & 0.841 & 0.583 & 10.762 \\
\hline & -0.626 & 1.258 & 11.464 \\
\hline N & -1.571 & 2.275 & 9.085 \\
\hline & -2.198 & 2.680 & 9.784 \\
\hline & -0.369 & 1.642 & 9.361 \\
\hline C & -1.720 & 2.332 & 7.741 \\
\hline $\mathrm{H}$ & -2.565 & 2.766 & 7.233 \\
\hline & -0.687 & 1.759 & 7.137 \\
\hline & 0.163 & 1.333 & 8.133 \\
\hline $\mathrm{H}$ & 1.098 & 0.853 & 7.893 \\
\hline C & 3.827 & -0.014 & 4.844 \\
\hline H & 4.551 & 0.756 & 4.545 \\
\hline $\mathrm{H}$ & 4.013 & -0.866 & 4.186 \\
\hline $\mathrm{C}$ & 2.407 & 0.485 & 4.451 \\
\hline $\mathrm{O}$ & 1.639 & 0.989 & 5.356 \\
\hline $\mathrm{O}$ & 2.156 & 0.373 & 3.238 \\
\hline $\mathrm{C}$ & 0.547 & 7.131 & 6.691 \\
\hline $\mathrm{H}$ & -0.337 & 7.543 & 6.197 \\
\hline $\mathrm{H}$ & 1.397 & 7.749 & 6.382 \\
\hline $\mathrm{N}$ & 1.971 & 5.042 & .351 \\
\hline $\mathrm{H}$ & 2.854 & 5.380 & 6.748 \\
\hline $\mathrm{C}$ & 0.755 & 5.709 & 6.273 \\
\hline C & 1.797 & 3.797 & 5.861 \\
\hline $\mathrm{H}$ & 2.555 & 3.032 & 5.850 \\
\hline $\mathrm{N}$ & 0.552 & 3.625 & 5.450 \\
\hline $\mathrm{C}$ & -0.107 & 4.808 & 5.701 \\
\hline $\mathrm{H}$ & -1.144 & 4.931 & 5.433 \\
\hline $\mathrm{C}$ & -4.324 & -2.792 & 4.042 \\
\hline $\mathrm{O}$ & -5.273 & -2.146 & 4.477 \\
\hline $\mathrm{N}$ & -3.022 & -2.475 & 3.818 \\
\hline C & -2.213 & -1.327 & 3.448 \\
\hline $\mathrm{H}$ & -2.783 & -0.401 & 3.523 \\
\hline $\mathrm{C}$ & -1.060 & -1.299 & 4.482 \\
\hline $\mathrm{O}$ & -0.403 & -2.348 & 4.634 \\
\hline $\mathrm{O}$ & -0.960 & -0.256 & 5.214 \\
\hline $\mathrm{C}$ & -1.792 & -1.578 & 1.935 \\
\hline $\mathrm{C}$ & -2.541 & -0.604 & 1.033 \\
\hline & -2.173 & 0.409 & 1.208 \\
\hline
\end{tabular}




\begin{tabular}{|c|c|c|c|}
\hline & -3.620 & -0.616 & \\
\hline & -2.366 & -0.856 & -0.016 \\
\hline & -0.286 & -1.472 & 1.675 \\
\hline & 0.248 & -2.324 & 2.092 \\
\hline & -0.101 & -1.467 & 0.596 \\
\hline & 0.115 & -0.544 & 2.096 \\
\hline & -2.381 & -3.321 & 1.476 \\
\hline & -2.655 & -3.753 & 3.230 \\
\hline & -1.785 & -4.227 & 3.679 \\
\hline & -4.061 & -4.269 & 3.648 \\
\hline 1 & -3.986 & -4.894 & 4.541 \\
\hline & -4.830 & -4.950 & 2.640 \\
\hline $\mathrm{H}$ & -5.442 & -4.422 & 2.034 \\
\hline & -4.266 & -6.122 & 2.190 \\
\hline 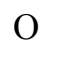 & -3.355 & -6.630 & 2.854 \\
\hline a & -4.731 & -6.728 & 0.898 \\
\hline $\mathrm{H}$ & -5.694 & -6.334 & 0.569 \\
\hline$H$ & -4.855 & -7.796 & 1.099 \\
\hline & -3.680 & -6.526 & -0.223 \\
\hline & -3.857 & -5.553 & -0.694 \\
\hline $\mathrm{H}$ & -2.679 & -6.498 & 0.219 \\
\hline$\smile$ & -3.720 & -7.643 & -1.273 \\
\hline H & -3.087 & -7.358 & -2.124 \\
\hline $\mathrm{H}$ & -3.265 & -8.543 & -0.856 \\
\hline$N$ & -5.811 & -6.824 & -2.304 \\
\hline $\mathrm{H}$ & -5.336 & -6.574 & -3.182 \\
\hline & -6.689 & -7.205 & -2.657 \\
\hline & -5.112 & -8.008 & -1.788 \\
\hline H & -5.709 & -8.398 & -0.949 \\
\hline 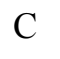 & -5.052 & -9.135 & -2.852 \\
\hline D & -4.073 & -9.930 & -2.797 \\
\hline $\mathrm{O}$ & -5.961 & -9.150 & -3.715 \\
\hline $\mathrm{Fe}$ & -0.362 & 1.633 & 5.042 \\
\hline $\mathrm{O}$ & -0.207 & 1.649 & 3.421 \\
\hline $\mathrm{C}$ & -3.060 & 2.588 & 4.084 \\
\hline $\mathrm{O}$ & -3.173 & 1.738 & 3.173 \\
\hline U & -2.127 & 2.626 & 4.972 \\
\hline C & -4.133 & 3.665 & 4.213 \\
\hline N & -5.075 & 3.144 & 4.416 \\
\hline $\mathrm{H}$ & -3.905 & 4.300 & 5.073 \\
\hline $\mathrm{C}$ & -4.333 & 4.507 & 2.942 \\
\hline 11 & -3.931 & 3.966 & 2.083 \\
\hline N & -3.772 & 5.441 & 2.985 \\
\hline C & -5.787 & 4.827 & 2.571 \\
\hline D & -6.715 & 4.023 & 2.882 \\
\hline
\end{tabular}


Coordinate of ${ }^{5} \mathrm{R}^{\prime}$

\begin{tabular}{|c|c|c|c|}
\hline & 00 & 0 & 0000 \\
\hline & 0.86500000 & 59000000 & 10.82200000 \\
\hline & -0.57000000 & 1.30900000 & 11.54800000 \\
\hline & -1.47500000 & .41600000 & 9.16100000 \\
\hline & -2.10400000 & 82100000 & 0000 \\
\hline & -0.33500000 & 0000 & 9.43 \\
\hline & -1.59800000 & 2.52400000 & 7.82000000 \\
\hline & -2.40900000 & 3.02200000 & 0000 \\
\hline & -0.60 & 1.89 & 0000 \\
\hline & 0000 & 1.357 & 0000 \\
\hline & 1.07500000 & 0.79500000 & 7.9 \\
\hline & 3.74800000 & -0.13 & 0000 \\
\hline & 4.58300000 & 0.43300000 & 0000 \\
\hline & 3.68600000 & -1.05300000 & 000 \\
\hline & 2.45600000 & 0000 & 000 \\
\hline & 1.59400000 & 0.90000000 & 000 \\
\hline & 2.35 & 000 & 000 \\
\hline & 0.55200000 & 7.13300000 & 6.6 \\
\hline & -0.34100000 & 7.53900000 & 6.1 \\
\hline & 1.3 & & \\
\hline & 1.99800000 & 5.06600000 & 6.2 \\
\hline & 2.88400000 & 5.40800000 & 6.65100000 \\
\hline & 0.77 & 5.71 & 00 \\
\hline & 1.825 & 3.82 & \\
\hline & 2.60900000 & 3.09100000 & 00000 \\
\hline & 0.57300000 & 3.63900000 & 5.38700000 \\
\hline & -0.092 & 00 & \\
\hline & -1.13 & 0000 & \\
\hline & -4.12000000 & -1.77000000 & 2.04000000 \\
\hline & -4.36200000 & -0.93500000 & 1.18200000 \\
\hline & -3.33500000 & -1.75700000 & \\
\hline & -2.08100000 & -1.16300000 & 3.56200000 \\
\hline & -1.96200000 & -0.26000000 & 2.97000000 \\
\hline & -2.16600000 & -0.82900000 & 5.06000000 \\
\hline & -3.14100000 & -1.27500000 & 5.69700000 \\
\hline$O$ & -1.22200000 & -0.14700000 & 5.57900000 \\
\hline & -0.99600000 & -2.23300000 & 3.17600000 \\
\hline & -0.54600000 & -1.93100000 & 1.74300000 \\
\hline & 0.01800000 & -0.99000000 & 1.73800000 \\
\hline & -1.39400000 & -1.82500000 & 1.06100000 \\
\hline & 0.07800000 & -2.74300000 & 1.36100000 \\
\hline & 0.22900000 & -2.38500000 & 4.13600000 \\
\hline
\end{tabular}




\begin{tabular}{|c|c|c|c|}
\hline & 000 & 000 & 00 \\
\hline & 0.92000000 & -3.15200000 & 3.77100000 \\
\hline & 0.75200000 & -1.42900000 & 4.18400000 \\
\hline & -1.88000000 & -3.91700000 & 3.23700000 \\
\hline & -3.53200000 & -3.15900000 & 3.48900000 \\
\hline & -3.89500000 & -3.33700000 & 4.49900000 \\
\hline & -4.59500000 & -3.21200000 & 2.35300000 \\
\hline & -5.59600000 & -3.24000000 & 2.78700000 \\
\hline & -4.50400000 & -4.22200000 & 1.34100000 \\
\hline & -3.84700000 & -4.11600000 & 0.58200000 \\
\hline & -4.91000000 & -5.48400000 & 1.70400000 \\
\hline & -5.43800000 & -5.64300000 & 2.80600000 \\
\hline & -4.78700000 & -6.59600000 & 0.69900000 \\
\hline & -5.75300000 & -6.62300000 & 0.18000000 \\
\hline & -4.70300000 & -7.52700000 & 1.26700000 \\
\hline & -3.70600000 & -6.48400000 & -0.37400000 \\
\hline & -3.88900000 & -5.58000000 & -0.96900000 \\
\hline & -2.71700000 & -6.39100000 & 0.09100000 \\
\hline & -3.70800000 & -7.69200000 & -1.31900000 \\
\hline & -3.0 & -7 & 000 \\
\hline & -3.26100000 & -8.55300000 & -0.81700000 \\
\hline & -5.81700000 & -6.90200000 & -2.32700000 \\
\hline & -5.36800000 & -6.65400000 & -3.22000000 \\
\hline & -6.71100000 & -7.27800000 & -2.64300000 \\
\hline & -5.10300000 & -8.08000000 & -1.82100000 \\
\hline & -5.68400000 & -8.47800000 & -0.97500000 \\
\hline & -5.04300000 & -9.19700000 & -2.88300000 \\
\hline & -4.05400000 & -9.97900000 & -2.82200000 \\
\hline & -5.94900000 & -9.21900000 & -3.74700000 \\
\hline & -0.40600000 & 1.63800000 & 5.13100000 \\
\hline & -0.36300000 & 1.45000000 & 3.51000000 \\
\hline & -3.08800000 & 2.52100000 & 4.24000000 \\
\hline & -3.25700000 & 1.52300000 & 3.52300000 \\
\hline & -2.09700000 & 2.70700000 & 5.05700000 \\
\hline & -4.13100000 & 3.63700000 & 4.20600000 \\
\hline & -5.04400000 & 3.22300000 & 4.65200000 \\
\hline & -3.80300000 & 4.47500000 & 4.82800000 \\
\hline & -4.45600000 & 4.09200000 & 2.77300000 \\
\hline & -4.40300000 & 3.20600000 & 2.13400000 \\
\hline & -3.72100000 & 4.81900000 & 2.41800000 \\
\hline & -5.86300000 & 4.66800000 & 2.58500000 \\
\hline & -6.85200000 & 3.97800000 & 2.98200000 \\
\hline & -5.96300000 & 5.78200000 & 1.99200000 \\
\hline
\end{tabular}

Coordinate of ${ }^{5} \mathrm{TS} \mathrm{a}^{\prime}$ 


\begin{tabular}{|c|c|c|c|}
\hline C & 0.18700000 & 1.49100000 & 10. \\
\hline $\mathrm{H}$ & 0.81800000 & 0.60000000 & 10.82700000 \\
\hline & -0.60100000 & 1.34100000 & 11.56100000 \\
\hline & -1.47900000 & 2.49000000 & 9.16700000 \\
\hline & -2.11100000 & 2.89600000 & 9.85900000 \\
\hline 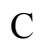 & -0.36300000 & 1.71700000 & 9.44300000 \\
\hline & -1.57600000 & 2.61500000 & 7.82200000 \\
\hline & -2.37300000 & 3.13600000 & 7.31700000 \\
\hline N & -0.59300000 & 1.96800000 & 7.22000000 \\
\hline & 0.16600000 & 1.40300000 & 8.21600000 \\
\hline & 1.04400000 & 0.81900000 & 7.98400000 \\
\hline $\mathrm{C}$ & 3.74800000 & -0.13200000 & 4.78400000 \\
\hline $\mathrm{H}$ & 4.58300000 & 0.43300000 & 4.35500000 \\
\hline $\mathrm{H}$ & 3.68600000 & -1.05300000 & 4.19200000 \\
\hline $\mathrm{C}$ & 2.45600000 & 0.64800000 & 4.46000000 \\
\hline $\mathrm{O}$ & 1.59400000 & 0000 & 0000 \\
\hline $\mathrm{O}$ & 2.35600000 & 0.96200000 & 3.24100000 \\
\hline C & 0.54800000 & 7.09300000 & 6.63000000 \\
\hline $\mathrm{H}$ & -0.34900000 & 7.49200000 & 6.14900000 \\
\hline $\mathrm{H}$ & 1.38100000 & 7.73000000 & 6.31100000 \\
\hline $\mathrm{N}$ & 2.00700000 & 5.03500000 & 6.21400000 \\
\hline $\mathrm{H}$ & 2.88900000 & 5.37400000 & 6.61000000 \\
\hline $\mathrm{C}$ & 0.77600000 & 5.68200000 & 000 \\
\hline $\mathrm{C}$ & 1.84500000 & 3.81100000 & 5.67000000 \\
\hline $\mathrm{H}$ & 2.63700000 & 3.08900000 & 5.58300000 \\
\hline $\mathrm{J}$ & 0.59400000 & 3.62600000 & 000 \\
\hline C & -0.07900000 & 4.78800000 & 5.59800000 \\
\hline $\mathrm{H}$ & -1.12400000 & 4.89700000 & 5.36300000 \\
\hline $\mathrm{C}$ & -4.21900000 & -1.81900000 & 2.19200000 \\
\hline $\mathrm{O}$ & -4.53300000 & -0.96100000 & 1.38600000 \\
\hline $\mathrm{N}$ & -3.43200000 & -1.79600000 & 3.31700000 \\
\hline $\mathrm{C}$ & -2.27600000 & -1.03500000 & 3.70600000 \\
\hline $\mathrm{H}$ & -2.32500000 & -0.05700000 & .23400000 \\
\hline $\mathrm{C}$ & -2.30700000 & -0.77500000 & 5.21100000 \\
\hline $\mathrm{O}$ & -3.23100000 & -1.29200000 & 5.86800000 \\
\hline $\mathrm{O}$ & -1.38300000 & -0.04100000 & 5.69000000 \\
\hline $\mathrm{C}$ & -1.00200000 & -1.82300000 & 3.20700000 \\
\hline $\mathrm{C}$ & -0.50100000 & -1.02400000 & 2.02200000 \\
\hline $\mathrm{H}$ & -0.28000000 & 0.10000000 & 2.58200000 \\
\hline $\mathrm{H}$ & -1.26100000 & -0.81100000 & 1.26800000 \\
\hline $\mathrm{H}$ & 0.44000000 & -1.33200000 & 1.57600000 \\
\hline $\mathrm{C}$ & 0.11100000 & -2.08100000 & 4.23100000 \\
\hline $\mathrm{H}$ & -0.28300000 & -2.62500000 & 5.09200000 \\
\hline $\mathrm{H}$ & 0.87900000 & -2.70300000 & 3.76500000 \\
\hline $\mathrm{H}$ & 0.56600000 & -1.15700000 & $\begin{array}{c}4.58500000 \\
\text { S17 }\end{array}$ \\
\hline
\end{tabular}




\begin{tabular}{|c|c|c|c|}
\hline S & -1.64100000 & -3.56200000 & 2.73300000 \\
\hline $\mathrm{C}$ & -3.32200000 & -3.23400000 & 3.41700000 \\
\hline $\mathrm{H}$ & -3.45100000 & -3.65300000 & 4.41500000 \\
\hline $\mathrm{C}$ & -4.50100000 & -3.32900000 & 2.41000000 \\
\hline $\mathrm{H}$ & -5.43200000 & -3.47900000 & 2.96000000 \\
\hline $\mathrm{N}$ & -4.46200000 & -4.27800000 & 1.34500000 \\
\hline $\mathrm{H}$ & -3.80000000 & -4.15400000 & 0.59300000 \\
\hline $\mathrm{C}$ & -4.90000000 & -5.55000000 & 1.64400000 \\
\hline $\mathrm{O}$ & -5.45500000 & -5.75900000 & 2.72300000 \\
\hline $\mathrm{C}$ & -4.76500000 & -6.61200000 & 0.59400000 \\
\hline $\mathrm{H}$ & -5.72900000 & -6.62300000 & 0.07200000 \\
\hline $\mathrm{H}$ & -4.68000000 & -7.56600000 & 1.12500000 \\
\hline $\mathrm{C}$ & -3.68000000 & -6.45800000 & -0.47000000 \\
\hline $\mathrm{H}$ & -3.86600000 & -5.54000000 & -1.04200000 \\
\hline $\mathrm{H}$ & -2.68900000 & -6.36900000 & -0.00600000 \\
\hline $\mathrm{C}$ & -3.68400000 & -7.64900000 & -1.43800000 \\
\hline $\mathrm{H}$ & -3.07700000 & -7.40900000 & -2.32000000 \\
\hline $\mathrm{H}$ & -3.19800000 & -8.50700000 & -0.96900000 \\
\hline $\mathrm{N}$ & -5.84000000 & -6.91600000 & -2.38900000 \\
\hline $\mathrm{H}$ & -5.41000000 & -6.66100000 & -3.28900000 \\
\hline $\mathrm{H}$ & -6.72900000 & -7.31700000 & -2.69000000 \\
\hline $\mathrm{C}$ & -5.08800000 & -8.06900000 & -1.88700000 \\
\hline $\mathrm{H}$ & -5.63200000 & -8.46600000 & -1.01600000 \\
\hline $\mathrm{C}$ & -5.03700000 & -9.21000000 & -2.91900000 \\
\hline $\mathrm{O}$ & -4.05400000 & -9.99700000 & -2.83100000 \\
\hline $\mathrm{O}$ & -5.94100000 & -9.24900000 & -3.78400000 \\
\hline $\mathrm{Fe}$ & -0.40800000 & 1.62200000 & 5.01700000 \\
\hline $\mathrm{O}$ & -0.40200000 & 1.18200000 & 3.27200000 \\
\hline $\mathrm{C}$ & -3.15400000 & 2.59800000 & 4.20700000 \\
\hline $\mathrm{O}$ & -3.47300000 & 1.56800000 & 3.59100000 \\
\hline $\mathrm{O}$ & -2.05400000 & 2.77900000 & 4.86400000 \\
\hline $\mathrm{C}$ & -4.13700000 & 3.76000000 & 4.19500000 \\
\hline $\mathrm{H}$ & -5.06000000 & 3.38200000 & 4.64900000 \\
\hline $\mathrm{H}$ & -3.76500000 & 4.58800000 & 4.80400000 \\
\hline $\mathrm{C}$ & -4.46800000 & 4.21900000 & 2.76300000 \\
\hline $\mathrm{H}$ & -4.39500000 & 3.34400000 & 2.11200000 \\
\hline $\mathrm{H}$ & -3.75300000 & 4.96900000 & 2.41400000 \\
\hline $\mathrm{C}$ & -5.88900000 & 4.75500000 & 2.58200000 \\
\hline $\mathrm{O}$ & -6.85700000 & 4.04100000 & 2.98800000 \\
\hline $\mathrm{O}$ & -6.01900000 & 5.86500000 & 1.98800000 \\
\hline
\end{tabular}

Coordinate of ${ }^{5} \mathrm{IM} 1 \mathrm{a}^{\prime}$

$\begin{array}{cccc}\mathrm{C} & 0.14700000 & 1.49100000 & 10.86600000 \\ \mathrm{H} & 0.78800000 & 0.60700000 & 10.86600000 \\ \mathrm{H} & -0.62900000 & 1.32900000 & 11.62100000 \\ & & & \mathrm{~S} 18\end{array}$




\begin{tabular}{|c|c|c|c|}
\hline & -1.56300000 & 2.45200000 & 000 \\
\hline $\mathrm{H}$ & -2.18600000 & 2.86100000 & 9.93500000 \\
\hline & -0.42700000 & 1.70400000 & 9.50000000 \\
\hline & -1.68900000 & 2.55400000 & 7.89000000 \\
\hline П & -2.50000000 & 3.06400000 & 7.39500000 \\
\hline & -0.70900000 & 1.91000000 & 7.27700000 \\
\hline & 0.08100000 & 1.37700000 & 8.26800000 \\
\hline $\mathrm{H}$ & 0.96800000 & 0.80800000 & 8.03000000 \\
\hline $\mathrm{C}$ & 3.74800000 & -0.13200000 & 4.78400000 \\
\hline & 4.58300000 & 0.43300000 & 4.35500000 \\
\hline $\mathrm{H}$ & 3.68600000 & -1.05300000 & 4.19200000 \\
\hline $\mathrm{C}$ & 2.45600000 & 0.64800000 & 4.46000000 \\
\hline ) & 1.59400000 & 0.90000000 & 5.36300000 \\
\hline $\mathrm{O}$ & 2.35600000 & 0.96200000 & 00000 \\
\hline $\mathrm{C}$ & 0.56900000 & 7.16000000 & 6.71300000 \\
\hline $\mathrm{H}$ & -0.31800000 & 7.57000000 & 6.22200000 \\
\hline $\mathrm{H}$ & 1.41600000 & 7.78500000 & 6.40800000 \\
\hline $\mathrm{N}$ & 2.01400000 & 5.09300000 & 6.30200000 \\
\hline $\mathrm{H}$ & 2.90800000 & 5.43700000 & 6.6670000 \\
\hline $\mathrm{C}$ & 0.78700000 & 5.74300000 & 6.28900000 \\
\hline $\mathrm{C}$ & 1.83000000 & 3.84600000 & 00000 \\
\hline $\mathrm{H}$ & 2.61200000 & 0000 & 0000 \\
\hline $\mathrm{N}$ & 0.56900000 & 3.65100000 & 5.48200000 \\
\hline $\mathrm{C}$ & -0.09000000 & 4.82800000 & 5.77000000 \\
\hline $\mathrm{H}$ & $-1.14 \mathrm{C}$ & 000 & 0000 \\
\hline $\mathrm{C}$ & -4.14000000 & -1.82400000 & 2.04100000 \\
\hline $\mathrm{O}$ & -4.37500000 & -1.02100000 & 1.15300000 \\
\hline $\mathrm{N}$ & -3.33700000 & -1.79100000 & 3.14600000 \\
\hline $\mathrm{C}$ & -2.07200000 & -1.20100000 & 3.52100000 \\
\hline $\mathrm{H}$ & -1.87600000 & -0.29200000 & 2.95300000 \\
\hline $\mathrm{C}$ & -2.11300000 & -0.83500000 & 5.01400000 \\
\hline $\mathrm{O}$ & -3.03200000 & -1.30200000 & 5.70700000 \\
\hline $\mathrm{O}$ & -1.15200000 & -0.120 & 5.46100000 \\
\hline $\mathrm{C}$ & -1.00000000 & -2.29500000 & 3.16500000 \\
\hline $\mathrm{C}$ & -0.61100000 & -2.16200000 & 1.75100000 \\
\hline $\mathrm{H}$ & 0.63700000 & 1.10400000 & 3.02600000 \\
\hline $\mathrm{H}$ & -1.26500000 & -1.66100000 & 1.04300000 \\
\hline $\mathrm{H}$ & 0.15200000 & -2.81800000 & 1.34500000 \\
\hline $\mathrm{C}$ & 0.19700000 & -2.40600000 & 4.10900000 \\
\hline $\mathrm{H}$ & -0.12100000 & -2.63500000 & 5.12500000 \\
\hline $\mathrm{H}$ & 0.85200000 & -3.21100000 & 3.76400000 \\
\hline $\mathrm{H}$ & 0.76000000 & -1.47100000 & 4.13100000 \\
\hline S & -1.95000000 & -4.00500000 & 3.32300000 \\
\hline $\mathrm{C}$ & -3.56900000 & -3.17800000 & 3.52900000 \\
\hline $\mathrm{H}$ & -3.93600000 & -3.30700000 & $\begin{array}{c}4.54500000 \\
\text { S19 }\end{array}$ \\
\hline
\end{tabular}




\begin{tabular}{|c|c|c|c|}
\hline & -4.63 & 0000 & 000 \\
\hline $\mathrm{H}$ & -5.63600000 & -3.26200000 & 2.82700000 \\
\hline & -4.54500000 & -4.26800000 & 1.39500000 \\
\hline & -3.87400000 & -4.17900000 & 64700000 \\
\hline & -5.00400000 & -5.51700000 & 1.73500000 \\
\hline U & -5.57200000 & -5.67300000 & .81800000 \\
\hline & -4.87900000 & 0000 & 0.72000000 \\
\hline & -5.83400000 & -6.63200000 & 0.18200000 \\
\hline$\pi$ & -4.81800000 & -7.56000000 & .27900000 \\
\hline & -3.77400000 & -6.51100000 & 0000 \\
\hline & -3.94400000 & -5.60700000 & -0.92800000 \\
\hline $\mathrm{H}$ & -2.79600000 & -6.41600000 & 0.16000000 \\
\hline $\mathrm{C}$ & -3.75100000 & -7.71800000 & -1.27400000 \\
\hline 1 & -3.07100000 & -7.51500000 & -2.10900000 \\
\hline $\mathrm{H}$ & -3.33000000 & -8.58200000 & 0000 \\
\hline & -5.81800000 & -6.91000000 & 0000 \\
\hline H & -5.33600000 & -6.65800000 & -3.23000000 \\
\hline 11 & -6.69800000 & -7.28400000 & -2.71200000 \\
\hline $\mathrm{C}$ & -5.13200000 & -8.09300000 & 00000 \\
\hline $\mathrm{H}$ & -5.74600000 & -8.48500000 & -0.99900000 \\
\hline$C$ & -5.04800000 & -9.20800000 & -2.88600000 \\
\hline $\mathrm{O}$ & -4.05100000 & -9.97800000 & -2.82200000 \\
\hline $\mathrm{O}$ & -5.94600000 & -9.23700000 & -3.75900000 \\
\hline $\mathrm{Fe}$ & -0.42000000 & 1.67800000 & 5.06600000 \\
\hline $\mathrm{O}$ & -0.22700000 & 1.52700000 & 3.21100000 \\
\hline $\mathrm{C}$ & -3.18100000 & 000 & 000 \\
\hline 0 & -3.43300000 & 1.36400000 & 3.83700000 \\
\hline $\mathrm{O}$ & -2.05400000 & 2.83800000 & 4.83800000 \\
\hline C & -4.22900000 & 3.58900000 & 4.17400000 \\
\hline H & -5.13700000 & 3.21000000 & 4.65800000 \\
\hline $\mathrm{H}$ & -3.89600000 & 4.47700000 & 4.71700000 \\
\hline C & -4.56800000 & 3.93400000 & 2.71200000 \\
\hline $\mathrm{H}$ & -4.60200000 & 2.99500000 & 2.15400000 \\
\hline $\mathrm{H}$ & -3.79400000 & 4.57200000 & 2.27900000 \\
\hline $\mathrm{C}$ & -5.93900000 & 4.59400000 & 2.55100000 \\
\hline $\mathrm{O}$ & -6.95500000 & 3.94500000 & 2.95500000 \\
\hline $\mathrm{O}$ & -5.99000000 & 5.73100000 & 1.99600000 \\
\hline \multicolumn{4}{|c|}{ Coordinate of ${ }^{5} \mathrm{TS} 2 \mathrm{a}^{\prime}$} \\
\hline $\mathrm{C}$ & 0.13500000 & 1.47700000 & 10.75700000 \\
\hline 11 & 0.77800000 & 0.59400000 & 10.75900000 \\
\hline $\mathrm{H}$ & -0.65800000 & 1.30100000 & 11.49200000 \\
\hline . & -1.55200000 & 2.45400000 & 9.11500000 \\
\hline$-\pi$ & -2.17500000 & 2.85900000 & 9.81500000 \\
\hline & -0.41400000 & 1.70600000 & 9.3830000 \\
\hline
\end{tabular}




\begin{tabular}{|c|c|c|c|}
\hline$C^{2}$ & -1.68 & 0000 & 000 \\
\hline $\mathrm{H}$ & -2.49100000 & 3.06100000 & 7.27200000 \\
\hline & -0.70200000 & 1.90700000 & 7.16700000 \\
\hline & 0.09700000 & 1.37500000 & 15300000 \\
\hline & 0.98100000 & 0.80900000 & 7.90600000 \\
\hline 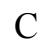 & 3.74800000 & -0.13200000 & 4.78400000 \\
\hline & 4.58300000 & 0.43300000 & 4.35500000 \\
\hline & 3.68600000 & -1.05300000 & 4.19200000 \\
\hline $\mathrm{C}$ & 2.45600000 & 0.64800000 & 4.46000000 \\
\hline $\mathrm{O}$ & 1.59400000 & 0.90000000 & 5.36300000 \\
\hline O & 2.35600000 & 0.96200000 & 3.24100000 \\
\hline $\mathrm{C}$ & 0.57900000 & 7.18700000 & 6.71800000 \\
\hline $\mathrm{H}$ & -0.30600000 & 7.59800000 & 6.22600000 \\
\hline $\mathrm{H}$ & 1.42700000 & 7.81500000 & 6.42200000 \\
\hline $\mathrm{N}$ & 2.02400000 & 5.11900000 & 6.30600000 \\
\hline $\mathrm{H}$ & 2.91800000 & 5.45800000 & 6.6740000 \\
\hline $\mathrm{C}$ & 0.79900000 & 5.77300000 & 6.28600000 \\
\hline C & 1.83600000 & 3.87100000 & 5.82400000 \\
\hline $\mathrm{H}$ & 2.61600000 & 3.13100000 & 5.75800000 \\
\hline $\mathrm{N}$ & 0.57600000 & 3.68000000 & 5.48100000 \\
\hline$C$ & -0.07800000 & 4.86000000 & 5.76400000 \\
\hline 1 & -1.13500000 & 4.96600000 & 5.57900000 \\
\hline $\mathrm{C}$ & -4.133 & -1.83400000 & 2.05200000 \\
\hline $\mathrm{O}$ & -4.36400000 & -1.01800000 & 1.17100000 \\
\hline$N$ & -3.30700000 & -1.82900000 & 3.13600000 \\
\hline $\mathrm{C}$ & -2.07000000 & -1.19100000 & 00000 \\
\hline $\mathrm{H}$ & -1.93700000 & -0.25800000 & 2.97200000 \\
\hline $\mathrm{C}$ & -2.11000000 & -0.84000000 & 5.02000000 \\
\hline $\mathrm{O}$ & -3.04200000 & -1.29900000 & .70300000 \\
\hline $\mathrm{O}$ & -1.13500000 & -0.15400000 & 5.47400000 \\
\hline $\mathrm{C}$ & -0.93400000 & -2.18300000 & 3.14300000 \\
\hline $\mathrm{C}$ & -0.64100000 & -2.18100000 & 1.76100000 \\
\hline H & 0.58300000 & 1.15600000 & 3.18700000 \\
\hline $\mathrm{H}$ & -1.31900000 & -1.72900000 & 1.04500000 \\
\hline $\mathrm{H}$ & 0.16200000 & -2.79500000 & 1.36600000 \\
\hline $\mathrm{C}$ & 0.21600000 & -2.39400000 & 4.10800000 \\
\hline $\mathrm{H}$ & -0.13700000 & -2.65700000 & 5.10200000 \\
\hline $\mathrm{H}$ & 0.85800000 & -3.20000000 & 3.74000000 \\
\hline $\mathrm{H}$ & 0.80200000 & -1.47600000 & 4.19500000 \\
\hline S & -2.02400000 & -4.14300000 & 3.34200000 \\
\hline $\mathrm{C}$ & -3.57200000 & -3.21100000 & 3.53100000 \\
\hline $\mathrm{H}$ & -3.94700000 & -3.30600000 & 4.55000000 \\
\hline 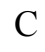 & -4.65000000 & -3.24900000 & 2.40300000 \\
\hline $\mathrm{H}$ & -5.65100000 & -3.25000000 & 2.83500000 \\
\hline 17 & -4.57200000 & -4.26400000 & 1.39500000 \\
\hline
\end{tabular}




\begin{tabular}{|c|c|c|c|}
\hline $\mathrm{H}$ & -3.89300000 & -4.17800000 & 000 \\
\hline $\mathrm{C}$ & -5.02100000 & -5.51600000 & 1.73600000 \\
\hline $\mathrm{O}$ & -5.59100000 & -5.67600000 & 2.81800000 \\
\hline $\mathrm{C}$ & -4.88800000 & -6.62000000 & 0.72100000 \\
\hline $\mathrm{H}$ & -5.84300000 & -6.63500000 & 0.18100000 \\
\hline $\mathrm{H}$ & -4.82500000 & -7.55800000 & 1.28200000 \\
\hline $\mathrm{C}$ & -3.78200000 & -6.50800000 & -0.32500000 \\
\hline $\mathrm{H}$ & -3.95200000 & -5.60400000 & -0.92500000 \\
\hline $\mathrm{H}$ & -2.80600000 & -6.41100000 & 0.16500000 \\
\hline $\mathrm{C}$ & -3.75600000 & -7.71500000 & -1.27100000 \\
\hline $\mathrm{H}$ & -3.07400000 & -7.51000000 & -2.10600000 \\
\hline $\mathrm{H}$ & -3.33300000 & -8.57900000 & -0.75300000 \\
\hline $\mathrm{N}$ & -5.82100000 & -6.91100000 & -2.36000000 \\
\hline $\mathrm{H}$ & -5.33700000 & -6.65900000 & -3.23300000 \\
\hline $\mathrm{H}$ & -6.69900000 & -7.28600000 & -2.71800000 \\
\hline $\mathrm{C}$ & -5.13400000 & -8.09300000 & -1.82500000 \\
\hline $\mathrm{H}$ & -5.75000000 & -8.48500000 & -1.00200000 \\
\hline $\mathrm{C}$ & -5.04800000 & -9.20800000 & -2.88700000 \\
\hline $\mathrm{O}$ & -4.05000000 & -9.97800000 & -2.82100000 \\
\hline $\mathrm{O}$ & -5.94600000 & -9.23900000 & -3.76000000 \\
\hline $\mathrm{Fe}$ & -0.44200000 & 1.69300000 & 5.14700000 \\
\hline $\mathrm{O}$ & -0.28500000 & 1.58400000 & 3.35500000 \\
\hline $\mathrm{C}$ & -3.16300000 & 2.49100000 & 4.29200000 \\
\hline $\mathrm{O}$ & -3.40900000 & 1.36700000 & 3.84200000 \\
\hline $\mathrm{O}$ & -2.05900000 & 2.84300000 & 4.89000000 \\
\hline $\mathrm{C}$ & -4.21400000 & 3.59000000 & 4.17700000 \\
\hline $\mathrm{H}$ & -5.11900000 & 3.21100000 & 4.66700000 \\
\hline $\mathrm{H}$ & -3.88400000 & 4.48400000 & 4.71400000 \\
\hline $\mathrm{C}$ & -4.55800000 & 3.92300000 & 2.71500000 \\
\hline $\mathrm{H}$ & -4.59500000 & 2.97800000 & 2.16500000 \\
\hline $\mathrm{H}$ & -3.78400000 & 4.55500000 & 2.27300000 \\
\hline $\mathrm{C}$ & -5.93000000 & 4.58400000 & 2.55300000 \\
\hline $\mathrm{O}$ & -6.94600000 & 3.93700000 & 2.95700000 \\
\hline $\mathrm{O}$ & -5.97900000 & 5.72000000 & 1.99500000 \\
\hline \multicolumn{4}{|c|}{ Coordinate of ${ }^{5} \mathrm{IM} 2 \mathrm{a}^{\prime}$} \\
\hline $\mathrm{C}$ & 0.16600000 & 1.50400000 & 10.87600000 \\
\hline $\mathrm{H}$ & 0.80700000 & 0.62100000 & 10.88700000 \\
\hline $\mathrm{H}$ & -0.61700000 & 1.35000000 & 11.62500000 \\
\hline $\mathrm{N}$ & -1.54800000 & 2.44200000 & 9.23900000 \\
\hline $\mathrm{H}$ & -2.17600000 & 2.84300000 & 9.94000000 \\
\hline $\mathrm{C}$ & -0.39800000 & 1.71500000 & 9.50700000 \\
\hline $\mathrm{C}$ & -1.66600000 & 2.55500000 & 7.89600000 \\
\hline $\mathrm{H}$ & -2.48300000 & 3.05000000 & 7.39600000 \\
\hline $\mathrm{N}$ & -0.66800000 & 1.93700000 & $\begin{array}{c}7.28400000 \\
\text { S22 }\end{array}$ \\
\hline
\end{tabular}




\begin{tabular}{|c|c|c|c|}
\hline $\mathrm{C}$ & 0.12700000 & 1.41100000 & 8.27600000 \\
\hline $\mathrm{H}$ & 1.02500000 & 0.86200000 & 8.03800000 \\
\hline $\mathrm{C}$ & 3.84000000 & -0.15000000 & 4.83400000 \\
\hline $\mathrm{H}$ & 4.58500000 & 0.58300000 & 4.50000000 \\
\hline $\mathrm{H}$ & 3.98600000 & -1.02600000 & 4.19400000 \\
\hline $\mathrm{C}$ & 2.44000000 & 0.40000000 & 4.47700000 \\
\hline $\mathrm{O}$ & 1.68100000 & 0.89800000 & 5.36300000 \\
\hline $\mathrm{O}$ & 2.17600000 & 0.31400000 & 3.24100000 \\
\hline $\mathrm{C}$ & 0.57500000 & 7.15100000 & 6.69500000 \\
\hline $\mathrm{H}$ & -0.31200000 & 7.55500000 & 6.19900000 \\
\hline $\mathrm{H}$ & 1.41900000 & 7.78200000 & 6.39300000 \\
\hline $\mathrm{N}$ & 2.00400000 & 5.05400000 & 6.37600000 \\
\hline $\mathrm{H}$ & 2.88300000 & 5.38400000 & 6.78700000 \\
\hline $\mathrm{C}$ & 0.79700000 & 5.73500000 & 6.26600000 \\
\hline $\mathrm{C}$ & 1.83300000 & 3.81600000 & 5.86700000 \\
\hline $\mathrm{H}$ & 2.58400000 & 3.04300000 & 5.87400000 \\
\hline $\mathrm{N}$ & 0.60000000 & 3.66100000 & 5.41400000 \\
\hline $\mathrm{C}$ & -0.05500000 & 4.85100000 & 5.65500000 \\
\hline $\mathrm{H}$ & -1.08500000 & 4.97800000 & 5.36300000 \\
\hline $\mathrm{C}$ & -4.03100000 & -1.81500000 & 2.05000000 \\
\hline $\mathrm{O}$ & -4.34300000 & -0.89300000 & 1.30900000 \\
\hline $\mathrm{N}$ & -3.04100000 & -1.96400000 & 2.96800000 \\
\hline $\mathrm{C}$ & -1.92400000 & -1.17200000 & 3.44200000 \\
\hline $\mathrm{H}$ & -1.90500000 & -0.23100000 & 2.88600000 \\
\hline $\mathrm{C}$ & -2.12800000 & -0.80100000 & 4.92200000 \\
\hline $\mathrm{O}$ & -3.11200000 & -1.26800000 & 5.52200000 \\
\hline $\mathrm{O}$ & -1.22500000 & -0.07500000 & 5.46100000 \\
\hline $\mathrm{C}$ & -0.64700000 & -1.97400000 & 3.18000000 \\
\hline $\mathrm{C}$ & -0.39700000 & -2.34000000 & 1.90600000 \\
\hline $\mathrm{H}$ & 0.63000000 & 0.99400000 & 3.09300000 \\
\hline $\mathrm{H}$ & -1.04000000 & -2.02300000 & 1.09000000 \\
\hline $\mathrm{H}$ & 0.45700000 & -2.95500000 & 1.65600000 \\
\hline $\mathrm{C}$ & 0.22800000 & -2.39800000 & 4.32500000 \\
\hline $\mathrm{H}$ & -0.37300000 & -2.91300000 & 5.08000000 \\
\hline $\mathrm{H}$ & 1.00100000 & -3.08500000 & 3.97700000 \\
\hline $\mathrm{H}$ & 0.68600000 & -1.53900000 & 4.81700000 \\
\hline S & -2.08000000 & -4.54800000 & 2.99500000 \\
\hline $\mathrm{C}$ & -3.38300000 & -3.33100000 & 3.36700000 \\
\hline $\mathrm{H}$ & -3.66600000 & -3.39400000 & 4.41800000 \\
\hline $\mathrm{C}$ & -4.58500000 & -3.22100000 & 2.36200000 \\
\hline $\mathrm{H}$ & -5.53600000 & -3.19700000 & 2.89700000 \\
\hline $\mathrm{N}$ & -4.66100000 & -4.17900000 & 1.29800000 \\
\hline $\mathrm{H}$ & -4.00500000 & -4.09700000 & 0.53300000 \\
\hline $\mathrm{C}$ & -5.06700000 & -5.44700000 & 1.64500000 \\
\hline $\mathrm{O}$ & -5.56300000 & -5.63600000 & $\begin{array}{c}2.75700000 \\
\text { S23 }\end{array}$ \\
\hline
\end{tabular}




$\begin{array}{lccc}\mathrm{C} & -4.97000000 & -6.53100000 & 0.60500000 \\ \mathrm{H} & -5.89900000 & -6.48700000 & 0.02300000 \\ \mathrm{H} & -4.97500000 & -7.48000000 & 1.14900000 \\ \mathrm{C} & -3.81000000 & -6.46100000 & -0.38900000 \\ \mathrm{H} & -3.91300000 & -5.55600000 & -1.00200000 \\ \mathrm{H} & -2.85800000 & -6.39500000 & 0.15100000 \\ \mathrm{C} & -3.78200000 & -7.68300000 & -1.32000000 \\ \mathrm{H} & -3.09400000 & -7.49300000 & -2.15300000 \\ \mathrm{H} & -3.36500000 & -8.54000000 & -0.78700000 \\ \mathrm{~N} & -5.84700000 & -6.91600000 & -2.44800000 \\ \mathrm{H} & -5.34300000 & -6.66300000 & -3.30900000 \\ \mathrm{H} & -6.70400000 & -7.31500000 & -2.83200000 \\ \mathrm{C} & -5.15500000 & -8.07700000 & -1.87600000 \\ \mathrm{H} & -5.77600000 & -8.45200000 & -1.04900000 \\ \mathrm{C} & -5.05200000 & -9.21700000 & -2.90900000 \\ \mathrm{O} & -4.05000000 & -9.97800000 & -2.82100000 \\ \mathrm{O} & -5.94200000 & -9.27300000 & -3.78900000 \\ \mathrm{Fe} & -0.45500000 & 1.72200000 & 5.07400000 \\ \mathrm{O} & -0.15600000 & 1.57100000 & 3.25100000 \\ \mathrm{C} & -3.16500000 & 2.51800000 & 4.21000000 \\ \mathrm{O} & -3.35100000 & 1.46000000 & 3.59500000 \\ \mathrm{O} & -2.12300000 & 2.78300000 & 4.94200000 \\ \mathrm{C} & -4.21700000 & 3.62000000 & 4.14400000 \\ \mathrm{H} & -5.10900000 & 3.23400000 & 4.65400000 \\ \mathrm{H} & -3.86700000 & 4.50100000 & 4.68800000 \\ \mathrm{C} & -4.60000000 & 3.96900000 & 2.69700000 \\ \mathrm{H} & -4.64300000 & 3.02900000 & 2.14100000 \\ \mathrm{H} & -3.84700000 & 4.61500000 & 2.23900000 \\ \mathrm{C} & -5.97300000 & 4.62700000 & 2.55900000 \\ \mathrm{O} & -6.98500000 & 3.96900000 & 2.95900000 \\ \mathrm{O} & -6.02900000 & 5.77000000 & 2.01800000\end{array}$

Coordinate of ${ }^{5} \mathrm{TS} 3 \mathrm{a}^{\prime}$

$\begin{array}{lrrr}\mathrm{C} & 0.14200000 & 1.50100000 & 10.77700000 \\ \mathrm{H} & 0.78000000 & 0.61500000 & 10.78100000 \\ \mathrm{H} & -0.65300000 & 1.33500000 & 11.51000000 \\ \mathrm{~N} & -1.54700000 & 2.47500000 & 9.13500000 \\ \mathrm{H} & -2.17400000 & 2.87400000 & 9.83600000 \\ \mathrm{C} & -0.40300000 & 1.73700000 & 9.40300000 \\ \mathrm{C} & -1.67200000 & 2.59100000 & 7.79500000 \\ \mathrm{H} & -2.48200000 & 3.08800000 & 7.28800000 \\ \mathrm{~N} & -0.67600000 & 1.96300000 & 7.18700000 \\ \mathrm{C} & 0.12100000 & 1.42700000 & 8.17300000 \\ \mathrm{H} & 1.01200000 & 0.87200000 & 7.92700000 \\ \mathrm{C} & 3.76900000 & -0.15800000 & 4.82500000\end{array}$




\begin{tabular}{|c|c|c|c|}
\hline $\mathrm{H}$ & 4.51400000 & 500000 & 4.46800000 \\
\hline $\mathrm{H}$ & 3.89400000 & -1.04100000 & 4.19100000 \\
\hline $\mathrm{C}$ & 2.36800000 & 0.40200000 & 4.48900000 \\
\hline $\mathrm{O}$ & 1.62300000 & 0.90100000 & 5.39000000 \\
\hline $\mathrm{O}$ & 2.08300000 & 0.32300000 & 3.25900000 \\
\hline $\mathrm{C}$ & 0.59300000 & 7.16300000 & 6.69500000 \\
\hline $\mathrm{H}$ & -0.29300000 & 7.56300000 & 6.19400000 \\
\hline $\mathrm{H}$ & 1.43600000 & 7.79900000 & 6.40200000 \\
\hline $\mathrm{N}$ & 2.03700000 & 5.07500000 & 6.37800000 \\
\hline $\mathrm{H}$ & 2.91400000 & 5.40600000 & 6.79300000 \\
\hline $\mathrm{C}$ & 0.82600000 & 5.74900000 & 6.26500000 \\
\hline $\mathrm{C}$ & 1.87300000 & 3.83500000 & 5.87100000 \\
\hline $\mathrm{H}$ & 2.63100000 & 3.06900000 & 5.88100000 \\
\hline $\mathrm{N}$ & 0.64200000 & 3.67200000 & 5.41600000 \\
\hline $\mathrm{C}$ & -0.01900000 & 4.85900000 & 5.65300000 \\
\hline $\mathrm{H}$ & -1.04900000 & 4.98000000 & 5.36000000 \\
\hline $\mathrm{C}$ & -4.00200000 & -1.79600000 & 2.06600000 \\
\hline $\mathrm{O}$ & -4.32800000 & -0.86600000 & 1.34200000 \\
\hline $\mathrm{N}$ & -3.00200000 & -1.95500000 & 2.97400000 \\
\hline $\mathrm{C}$ & -1.88500000 & -1.18200000 & 3.47900000 \\
\hline $\mathrm{H}$ & -1.80800000 & -0.24200000 & 2.92100000 \\
\hline $\mathrm{C}$ & -2.13000000 & -0.79600000 & 4.95400000 \\
\hline $\mathrm{O}$ & -3.13700000 & -1.26100000 & 5.52100000 \\
\hline $\mathrm{O}$ & -1.23500000 & -0.08800000 & 5.52100000 \\
\hline $\mathrm{C}$ & -0.64600000 & -2.05300000 & 3.29700000 \\
\hline $\mathrm{C}$ & -0.56200000 & -2.79700000 & 2.14400000 \\
\hline $\mathrm{H}$ & 0.53500000 & 1.03300000 & 3.22800000 \\
\hline $\mathrm{H}$ & -1.15700000 & -2.55800000 & 1.27100000 \\
\hline $\mathrm{H}$ & 0.31300000 & -3.41200000 & 1.97300000 \\
\hline $\mathrm{C}$ & 0.26100000 & -2.37100000 & 4.44200000 \\
\hline $\mathrm{H}$ & -0.30500000 & -2.92600000 & 5.20100000 \\
\hline $\mathrm{H}$ & 1.08100000 & -3.00800000 & 4.11000000 \\
\hline $\mathrm{H}$ & 0.64600000 & -1.47800000 & 4.93100000 \\
\hline $\mathrm{S}$ & -2.03400000 & -4.55200000 & 2.92800000 \\
\hline $\mathrm{C}$ & -3.33200000 & -3.33100000 & 3.34800000 \\
\hline $\mathrm{H}$ & -3.60400000 & -3.42000000 & 4.39900000 \\
\hline $\mathrm{C}$ & -4.55000000 & -3.21000000 & 2.36800000 \\
\hline $\mathrm{H}$ & -5.49200000 & -3.18700000 & 2.91700000 \\
\hline $\mathrm{N}$ & -4.64900000 & -4.16400000 & 1.30100000 \\
\hline $\mathrm{H}$ & -3.97700000 & -4.10500000 & 0.54800000 \\
\hline $\mathrm{C}$ & -5.07200000 & -5.42800000 & 1.65100000 \\
\hline $\mathrm{O}$ & -5.58100000 & -5.61100000 & 2.75800000 \\
\hline $\mathrm{C}$ & -4.97900000 & -6.51600000 & 0.61300000 \\
\hline $\mathrm{H}$ & -5.90600000 & -6.46700000 & 0.02800000 \\
\hline $\mathrm{H}$ & -4.99300000 & -7.46400000 & $\begin{array}{c}1.16000000 \\
\text { S25 }\end{array}$ \\
\hline
\end{tabular}




$\begin{array}{lrrr}\mathrm{C} & -3.81500000 & -6.45900000 & -0.37700000 \\ \mathrm{H} & -3.90800000 & -5.55600000 & -0.99300000 \\ \mathrm{H} & -2.86700000 & -6.39600000 & 0.17100000 \\ \mathrm{C} & -3.78600000 & -7.68400000 & -1.30300000 \\ \mathrm{H} & -3.09100000 & -7.50100000 & -2.13200000 \\ \mathrm{H} & -3.37500000 & -8.54200000 & -0.76400000 \\ \mathrm{~N} & -5.84400000 & -6.91200000 & -2.44200000 \\ \mathrm{H} & -5.33700000 & -6.66100000 & -3.30200000 \\ \mathrm{H} & -6.70100000 & -7.31000000 & -2.82800000 \\ \mathrm{C} & -5.15700000 & -8.07500000 & -1.86800000 \\ \mathrm{H} & -5.78300000 & -8.44900000 & -1.04400000 \\ \mathrm{C} & -5.05200000 & -9.21300000 & -2.90300000 \\ \mathrm{O} & -4.04900000 & -9.97300000 & -2.81800000 \\ \mathrm{O} & -5.94300000 & -9.27000000 & -3.78300000 \\ \mathrm{Fe} & -0.44200000 & 1.72600000 & 5.16800000 \\ \mathrm{O} & -0.25100000 & 1.61000000 & 3.38600000 \\ \mathrm{C} & -3.11700000 & 2.51500000 & 4.22000000 \\ \mathrm{O} & -3.30200000 & 1.45700000 & 3.60300000 \\ \mathrm{O} & -2.09600000 & 2.79000000 & 4.97300000 \\ \mathrm{C} & -4.17700000 & 3.61700000 & 4.15000000 \\ \mathrm{H} & -5.06400000 & 3.22900000 & 4.66800000 \\ \mathrm{H} & -3.83000000 & 4.50300000 & 4.68700000 \\ \mathrm{C} & -4.57200000 & 3.95700000 & 2.70400000 \\ \mathrm{H} & -4.62000000 & 3.01300000 & 2.15600000 \\ \mathrm{H} & -3.82200000 & 4.59900000 & 2.23400000 \\ \mathrm{C} & -5.94500000 & 4.61700000 & 2.56400000 \\ \mathrm{O} & -6.95800000 & 3.96300000 & 2.96600000 \\ \mathrm{O} & -6.00100000 & 5.75800000 & 2.01600000\end{array}$

Coordinate of ${ }^{5} \mathrm{IM} 3 \mathrm{a}^{\prime}$

$\begin{array}{rrrr}\mathrm{C} & 0.13400000 & 1.51000000 & 10.78700000 \\ \mathrm{H} & 0.77000000 & 0.62200000 & 10.79100000 \\ \mathrm{H} & -0.66300000 & 1.34300000 & 11.51700000 \\ \mathrm{~N} & -1.55200000 & 2.48600000 & 9.14200000 \\ \mathrm{H} & -2.18200000 & 2.88000000 & 9.84400000 \\ \mathrm{C} & -0.40600000 & 1.75100000 & 9.41100000 \\ \mathrm{C} & -1.66900000 & 2.61100000 & 7.80200000 \\ \mathrm{H} & -2.48000000 & 3.10600000 & 7.29400000 \\ \mathrm{~N} & -0.66600000 & 1.99300000 & 7.19600000 \\ \mathrm{C} & 0.12700000 & 1.45400000 & 8.18200000 \\ \mathrm{H} & 1.02100000 & 0.90400000 & 7.93700000 \\ \mathrm{C} & 3.74300000 & -0.20600000 & 4.84400000 \\ \mathrm{H} & 4.49700000 & 0.49700000 & 4.46600000 \\ \mathrm{H} & 3.84800000 & -1.10500000 & 4.22800000 \\ \mathrm{C} & 2.35000000 & 0.37000000 & 4.50200000\end{array}$




\begin{tabular}{|c|c|c|c|}
\hline $\mathrm{O}$ & 1.62200000 & 0.90700000 & 5.39700000 \\
\hline $\mathrm{O}$ & 2.05700000 & 0.26900000 & 3.27600000 \\
\hline $\mathrm{C}$ & 0.60200000 & 7.17800000 & 6.70800000 \\
\hline $\mathrm{H}$ & -0.28200000 & 7.57900000 & 6.20600000 \\
\hline $\mathrm{H}$ & 1.44600000 & 7.81500000 & 6.42000000 \\
\hline $\mathrm{N}$ & 2.04800000 & 5.09200000 & 6.38700000 \\
\hline $\mathrm{H}$ & 2.92400000 & 5.42000000 & 6.80500000 \\
\hline $\mathrm{C}$ & 0.83700000 & 5.76600000 & 6.27400000 \\
\hline $\mathrm{C}$ & 1.88400000 & 3.85300000 & 5.87500000 \\
\hline $\mathrm{H}$ & 2.64300000 & 3.08700000 & 5.88300000 \\
\hline $\mathrm{N}$ & 0.65500000 & 3.69200000 & 5.41700000 \\
\hline $\mathrm{C}$ & -0.00600000 & 4.87800000 & 5.65600000 \\
\hline $\mathrm{H}$ & -1.03600000 & 4.99900000 & 5.36200000 \\
\hline $\mathrm{C}$ & -3.88200000 & -1.75600000 & 2.03500000 \\
\hline $\mathrm{O}$ & -4.21100000 & -0.81600000 & 1.32600000 \\
\hline $\mathrm{N}$ & -2.89100000 & -1.93400000 & 2.95100000 \\
\hline $\mathrm{C}$ & -1.77400000 & -1.18600000 & 3.50300000 \\
\hline $\mathrm{H}$ & -1.63900000 & -0.26300000 & 2.92700000 \\
\hline $\mathrm{C}$ & -2.10300000 & -0.77300000 & 4.95500000 \\
\hline $\mathrm{O}$ & -3.12600000 & -1.25300000 & 5.48400000 \\
\hline $\mathrm{O}$ & -1.25300000 & -0.04200000 & 5.55800000 \\
\hline $\mathrm{C}$ & -0.56400000 & -2.11000000 & 3.43200000 \\
\hline $\mathrm{C}$ & -0.62800000 & -3.15800000 & 2.39700000 \\
\hline $\mathrm{H}$ & 0.54000000 & 1.03000000 & 3.24000000 \\
\hline $\mathrm{H}$ & -0.93800000 & -2.79100000 & 1.41600000 \\
\hline $\mathrm{H}$ & 0.31500000 & -3.69700000 & 2.29700000 \\
\hline $\mathrm{C}$ & 0.29800000 & -2.37800000 & 4.61900000 \\
\hline $\mathrm{H}$ & -0.23000000 & -3.03300000 & 5.33100000 \\
\hline $\mathrm{H}$ & 1.20700000 & -2.90700000 & 4.32500000 \\
\hline $\mathrm{H}$ & 0.56100000 & -1.47000000 & 5.15800000 \\
\hline S & -1.88900000 & -4.48900000 & 2.90300000 \\
\hline $\mathrm{C}$ & -3.24200000 & -3.30000000 & 3.30800000 \\
\hline $\mathrm{H}$ & -3.52400000 & -3.41200000 & 4.35400000 \\
\hline $\mathrm{C}$ & -4.43900000 & -3.17100000 & 2.31100000 \\
\hline $\mathrm{H}$ & -5.38900000 & -3.14600000 & 2.84700000 \\
\hline $\mathrm{N}$ & -4.52400000 & -4.11500000 & 1.23400000 \\
\hline $\mathrm{H}$ & -3.90800000 & -4.00800000 & 0.44000000 \\
\hline $\mathrm{C}$ & -4.89300000 & -5.39300000 & 1.58900000 \\
\hline $\mathrm{O}$ & -5.34000000 & -5.59000000 & 2.72000000 \\
\hline $\mathrm{C}$ & -4.81800000 & -6.47400000 & 0.54700000 \\
\hline $\mathrm{H}$ & -5.78500000 & -6.46800000 & 0.02800000 \\
\hline $\mathrm{H}$ & -4.75300000 & -7.42000000 & 1.09200000 \\
\hline $\mathrm{C}$ & -3.73100000 & -6.36900000 & -0.52400000 \\
\hline $\mathrm{H}$ & -3.91100000 & -5.47700000 & -1.13700000 \\
\hline $\mathrm{H}$ & -2.74600000 & -6.25400000 & $\begin{array}{c}-0.05500000 \\
\text { S27 }\end{array}$ \\
\hline
\end{tabular}




$\begin{array}{llll}\mathrm{C} & -3.72500000 & -7.60200000 & -1.43900000 \\ \mathrm{H} & -3.09400000 & -7.40700000 & -2.31500000 \\ \mathrm{H} & -3.25900000 & -8.44500000 & -0.92200000 \\ \mathrm{~N} & -5.86400000 & -6.89900000 & -2.46000000 \\ \mathrm{H} & -5.40000000 & -6.64700000 & -3.34400000 \\ \mathrm{H} & -6.72900000 & -7.32100000 & -2.80000000 \\ \mathrm{C} & -5.11900000 & -8.03600000 & -1.90800000 \\ \mathrm{H} & -5.68300000 & -8.40800000 & -1.04000000 \\ \mathrm{C} & -5.04400000 & -9.19900000 & -2.91800000 \\ \mathrm{O} & -4.05400000 & -9.97400000 & -2.81700000 \\ \mathrm{O} & -5.94200000 & -9.26100000 & -3.78900000 \\ \mathrm{Fe} & -0.43000000 & 1.74900000 & 5.17600000 \\ \mathrm{O} & -0.23200000 & 1.62700000 & 3.39600000 \\ \mathrm{C} & -3.09700000 & 2.52800000 & 4.21500000 \\ \mathrm{O} & -3.26800000 & 1.46800000 & 3.59700000 \\ \mathrm{O} & -2.08300000 & 2.81400000 & 4.97300000 \\ \mathrm{C} & -4.16700000 & 3.62100000 & 4.13900000 \\ \mathrm{H} & -5.04700000 & 3.23400000 & 4.66900000 \\ \mathrm{H} & -3.82200000 & 4.51600000 & 4.66500000 \\ \mathrm{C} & -4.57100000 & 3.94300000 & 2.69200000 \\ \mathrm{H} & -4.62600000 & 2.99100000 & 2.16000000 \\ \mathrm{H} & -3.82000000 & 4.57300000 & 2.20800000 \\ \mathrm{C} & -5.94100000 & 4.60900000 & 2.55300000 \\ \mathrm{O} & -6.95700000 & 3.95700000 & 2.95100000 \\ \mathrm{O} & -5.99300000 & 5.75300000 & 2.01200000\end{array}$

Coordinate of ${ }^{5} \mathrm{TS} 4 \mathrm{a}^{\prime}$

$\begin{array}{lrrr}\mathrm{C} & 0.13700000 & 1.51900000 & 10.88800000 \\ \mathrm{H} & 0.76800000 & 0.62700000 & 10.88700000 \\ \mathrm{H} & -0.64500000 & 1.36400000 & 11.63800000 \\ \mathrm{~N} & -1.57300000 & 2.48900000 & 9.26200000 \\ \mathrm{H} & -2.20400000 & 2.87800000 & 9.96700000 \\ \mathrm{C} & -0.42500000 & 1.75700000 & 9.52200000 \\ \mathrm{C} & -1.67600000 & 2.63800000 & 7.91800000 \\ \mathrm{H} & -2.48900000 & 3.14100000 & 7.42000000 \\ \mathrm{~N} & -0.66900000 & 2.04000000 & 7.30200000 \\ \mathrm{C} & 0.11200000 & 1.48700000 & 8.28800000 \\ \mathrm{H} & 1.01200000 & 0.94300000 & 8.04700000 \\ \mathrm{C} & 3.83200000 & -0.17000000 & 4.78500000 \\ \mathrm{H} & 4.58200000 & 0.56500000 & 4.46400000 \\ \mathrm{H} & 3.99100000 & -1.04700000 & 4.15000000 \\ \mathrm{C} & 2.43300000 & 0.37800000 & 4.41400000 \\ \mathrm{O} & 1.70600000 & 0.94200000 & 5.28100000 \\ \mathrm{O} & 2.13500000 & 0.21100000 & 3.19200000 \\ \mathrm{C} & 0.58200000 & 7.17100000 & 6.70900000\end{array}$




\begin{tabular}{|c|c|c|c|}
\hline $\mathrm{H}$ & -0.30400000 & 7.58100000 & 6.21600000 \\
\hline $\mathrm{H}$ & 1.42600000 & 7.80800000 & 6.41700000 \\
\hline $\mathrm{N}$ & 2.02000000 & 5.08600000 & 6.35600000 \\
\hline $\mathrm{H}$ & 2.89400000 & 5.40600000 & 6.78400000 \\
\hline $\mathrm{C}$ & 0.81000000 & 5.76500000 & 6.25500000 \\
\hline $\mathrm{C}$ & 1.86400000 & 3.87200000 & 5.78800000 \\
\hline $\mathrm{H}$ & 2.62400000 & 3.10800000 & 5.76900000 \\
\hline $\mathrm{N}$ & 0.63900000 & 3.72600000 & 5.30800000 \\
\hline $\mathrm{C}$ & -0.02600000 & 4.90200000 & 5.59300000 \\
\hline $\mathrm{H}$ & -1.05600000 & 5.02900000 & 5.30300000 \\
\hline $\mathrm{C}$ & -3.70700000 & -1.60700000 & 1.96500000 \\
\hline $\mathrm{O}$ & -4.01200000 & -0.65100000 & 1.27700000 \\
\hline $\mathrm{N}$ & -2.73000000 & -1.84100000 & 2.89500000 \\
\hline $\mathrm{C}$ & -1.64500000 & -1.20100000 & 3.60900000 \\
\hline $\mathrm{H}$ & -1.26800000 & -0.14300000 & 3.19900000 \\
\hline $\mathrm{C}$ & -2.10100000 & -0.80000000 & 5.05900000 \\
\hline $\mathrm{O}$ & -3.17600000 & -1.29200000 & 5.45900000 \\
\hline $\mathrm{O}$ & -1.29800000 & -0.09700000 & 5.72500000 \\
\hline $\mathrm{C}$ & -0.53000000 & -2.14300000 & 3.61000000 \\
\hline $\mathrm{C}$ & -0.53800000 & -3.21200000 & 2.58800000 \\
\hline $\mathrm{H}$ & 0.48100000 & 0.72400000 & 3.08700000 \\
\hline $\mathrm{H}$ & -0.75300000 & -2.82700000 & 1.58900000 \\
\hline $\mathrm{H}$ & 0.39300000 & -3.77800000 & 2.56000000 \\
\hline $\mathrm{C}$ & 0.48400000 & -2.21400000 & 4.69000000 \\
\hline $\mathrm{H}$ & 0.16500000 & -2.99100000 & 5.40300000 \\
\hline $\mathrm{H}$ & 1.45400000 & -2.53400000 & 4.31300000 \\
\hline $\mathrm{H}$ & 0.57200000 & -1.28400000 & 5.24300000 \\
\hline S & -1.88600000 & -4.44400000 & 3.02000000 \\
\hline $\mathrm{C}$ & -3.19900000 & -3.17900000 & 3.26400000 \\
\hline $\mathrm{H}$ & -3.55100000 & -3.22800000 & 4.29000000 \\
\hline $\mathrm{C}$ & -4.32500000 & -3.00600000 & 2.20800000 \\
\hline $\mathrm{H}$ & -5.29600000 & -2.93100000 & 2.70200000 \\
\hline $\mathrm{N}$ & -4.39800000 & -3.96700000 & 1.14200000 \\
\hline $\mathrm{H}$ & -3.84900000 & -3.84000000 & 0.30400000 \\
\hline $\mathrm{C}$ & -4.73900000 & -5.24300000 & 1.53200000 \\
\hline $\mathrm{O}$ & -5.11300000 & -5.41700000 & 2.69400000 \\
\hline $\mathrm{C}$ & -4.72100000 & -6.34800000 & 0.51300000 \\
\hline $\mathrm{H}$ & -5.69600000 & -6.33000000 & 0.01000000 \\
\hline $\mathrm{H}$ & -4.67100000 & -7.28100000 & 1.08200000 \\
\hline $\mathrm{C}$ & -3.65100000 & -6.30600000 & -0.58100000 \\
\hline $\mathrm{H}$ & -3.82300000 & -5.43400000 & -1.22400000 \\
\hline $\mathrm{H}$ & -2.65600000 & -6.19600000 & -0.13600000 \\
\hline $\mathrm{C}$ & -3.68300000 & -7.57400000 & -1.44900000 \\
\hline $\mathrm{H}$ & -3.06400000 & -7.42100000 & -2.34100000 \\
\hline $\mathrm{H}$ & -3.22000000 & -8.40400000 & $\begin{array}{r}-0.90800000 \\
\text { S29 }\end{array}$ \\
\hline
\end{tabular}




$\begin{array}{llll}\mathrm{N} & -5.84700000 & -6.86800000 & -2.41500000 \\ \mathrm{H} & -5.40900000 & -6.62600000 & -3.31500000 \\ \mathrm{H} & -6.72400000 & -7.28900000 & -2.72400000 \\ \mathrm{C} & -5.09000000 & -8.00500000 & -1.88000000 \\ \mathrm{H} & -5.63200000 & -8.37400000 & -0.99600000 \\ \mathrm{C} & -5.04100000 & -9.17100000 & -2.89300000 \\ \mathrm{O} & -4.05800000 & -9.95700000 & -2.80700000 \\ \mathrm{O} & -5.95000000 & -9.22100000 & -3.75300000 \\ \mathrm{Fe} & -0.47800000 & 1.75100000 & 5.11400000 \\ \mathrm{O} & -0.39100000 & 1.16700000 & 3.23200000 \\ \mathrm{C} & -3.16900000 & 2.54400000 & 4.23100000 \\ \mathrm{O} & -3.33900000 & 1.44300000 & 3.68300000 \\ \mathrm{O} & -2.12500000 & 2.89000000 & 4.91000000 \\ \mathrm{C} & -4.26100000 & 3.60700000 & 4.12200000 \\ \mathrm{H} & -5.13700000 & 3.22400000 & 4.66100000 \\ \mathrm{H} & -3.93100000 & 4.52400000 & 4.61800000 \\ \mathrm{C} & -4.66400000 & 3.87600000 & 2.66400000 \\ \mathrm{H} & -4.74400000 & 2.90300000 & 2.17100000 \\ \mathrm{H} & -3.89800000 & 4.46700000 & 2.15300000 \\ \mathrm{C} & -6.01500000 & 4.57500000 & 2.52200000 \\ \mathrm{O} & -7.04700000 & 3.93300000 & 2.89700000 \\ \mathrm{O} & -6.03900000 & 5.73500000 & 2.01500000\end{array}$

Coordinate of ${ }^{5} \mathrm{~Pa}^{\prime}$

$\begin{array}{rrrr}\mathrm{C} & 0.16200000 & 1.52500000 & 11.09800000 \\ \mathrm{H} & 0.79600000 & 0.63700000 & 11.11300000 \\ \mathrm{H} & -0.58900000 & 1.40400000 & 11.88400000 \\ \mathrm{~N} & -1.61100000 & 2.40700000 & 9.48400000 \\ \mathrm{H} & -2.23500000 & 2.81600000 & 10.18700000 \\ \mathrm{C} & -0.45200000 & 1.69400000 & 9.74600000 \\ \mathrm{C} & -1.73700000 & 2.52000000 & 8.14000000 \\ \mathrm{H} & -2.5600000 & 3.00500000 & 7.63900000 \\ \mathrm{~N} & -0.73400000 & 1.91300000 & 7.52800000 \\ \mathrm{C} & 0.06700000 & 1.39400000 & 8.51300000 \\ \mathrm{H} & 0.97200000 & 0.85500000 & 8.27500000 \\ \mathrm{C} & 3.76000000 & -0.18900000 & 4.75000000 \\ \mathrm{H} & 4.53800000 & 0.49800000 & 4.39400000 \\ \mathrm{H} & 3.87000000 & -1.09000000 & 4.13700000 \\ \mathrm{C} & 2.38300000 & 0.42300000 & 4.38200000 \\ \mathrm{O} & 1.65400000 & 0.92200000 & 5.28100000 \\ \mathrm{O} & 2.11800000 & 0.38200000 & 3.13700000 \\ \mathrm{C} & 0.56200000 & 7.18000000 & 6.73900000 \\ \mathrm{H} & -0.32400000 & 7.59000000 & 6.24800000 \\ \mathrm{H} & 1.41000000 & 7.80500000 & 6.43300000 \\ \mathrm{~N} & 1.99200000 & 5.09300000 & 6.36900000 \\ & & & 530\end{array}$




\begin{tabular}{|c|c|c|c|}
\hline $\mathrm{H}$ & 2.88300000 & 5.42700000 & 6.74800000 \\
\hline $\mathrm{C}$ & 0.77300000 & 5.76300000 & 6.31200000 \\
\hline $\mathrm{C}$ & 1.80600000 & 3.85300000 & 5.87300000 \\
\hline $\mathrm{H}$ & 2.57200000 & 3.09600000 & 5.82300000 \\
\hline $\mathrm{N}$ & 0.55400000 & 3.68100000 & 5.48600000 \\
\hline $\mathrm{C}$ & -0.09700000 & 4.86700000 & 5.75200000 \\
\hline $\mathrm{H}$ & -1.14200000 & 4.98000000 & 5.50900000 \\
\hline $\mathrm{C}$ & -3.76100000 & -1.88200000 & 2.13400000 \\
\hline $\mathrm{O}$ & -3.94900000 & -0.88200000 & 1.46200000 \\
\hline $\mathrm{N}$ & -2.87100000 & -2.19200000 & 3.12400000 \\
\hline $\mathrm{C}$ & -1.80900000 & -1.65200000 & 3.88500000 \\
\hline $\mathrm{H}$ & -0.06700000 & 0.60200000 & 3.07900000 \\
\hline $\mathrm{C}$ & -2.24500000 & -0.81400000 & 5.06700000 \\
\hline $\mathrm{O}$ & -3.42100000 & -1.03600000 & 5.44700000 \\
\hline $\mathrm{O}$ & -1.38900000 & -0.07800000 & 5.63300000 \\
\hline $\mathrm{C}$ & -0.67100000 & -2.38000000 & 3.80200000 \\
\hline $\mathrm{C}$ & -0.63800000 & -3.49400000 & 2.79100000 \\
\hline $\mathrm{H}$ & -1.60000000 & 1.29900000 & 3.24500000 \\
\hline $\mathrm{H}$ & -0.74100000 & -3.10700000 & 1.77500000 \\
\hline $\mathrm{H}$ & 0.29200000 & -4.06100000 & 2.83700000 \\
\hline $\mathrm{C}$ & 0.47400000 & -2.27200000 & 4.75600000 \\
\hline $\mathrm{H}$ & 0.44600000 & -3.12500000 & 5.44500000 \\
\hline $\mathrm{H}$ & 1.43200000 & -2.32300000 & 4.24200000 \\
\hline $\mathrm{H}$ & 0.42700000 & -1.35400000 & 5.33300000 \\
\hline $\mathrm{S}$ & -2.00400000 & -4.74900000 & 3.06100000 \\
\hline $\mathrm{C}$ & -3.35400000 & -3.54500000 & 3.36600000 \\
\hline $\mathrm{H}$ & -3.74800000 & -3.68100000 & 4.37400000 \\
\hline $\mathrm{C}$ & -4.43700000 & -3.26900000 & 2.27800000 \\
\hline $\mathrm{H}$ & -5.42000000 & -3.19100000 & 2.74600000 \\
\hline $\mathrm{N}$ & -4.50500000 & -4.16700000 & 1.16300000 \\
\hline $\mathrm{H}$ & -3.86900000 & -4.04700000 & 0.38800000 \\
\hline $\mathrm{C}$ & -4.91700000 & -5.44200000 & 1.47900000 \\
\hline $\mathrm{O}$ & -5.39100000 & -5.65300000 & 2.59600000 \\
\hline $\mathrm{C}$ & -4.83900000 & -6.49900000 & 0.42000000 \\
\hline $\mathrm{H}$ & -5.80500000 & -6.48700000 & -0.10100000 \\
\hline $\mathrm{H}$ & -4.77300000 & -7.45600000 & 0.94600000 \\
\hline $\mathrm{C}$ & -3.74700000 & -6.36700000 & -0.64200000 \\
\hline $\mathrm{H}$ & -3.92700000 & -5.46500000 & -1.23900000 \\
\hline $\mathrm{H}$ & -2.76300000 & -6.25900000 & -0.16900000 \\
\hline $\mathrm{C}$ & -3.74000000 & -7.58700000 & -1.57100000 \\
\hline $\mathrm{H}$ & -3.13800000 & -7.37100000 & -2.46300000 \\
\hline $\mathrm{H}$ & -3.24700000 & -8.42700000 & -1.07700000 \\
\hline $\mathrm{N}$ & -5.90800000 & -6.93400000 & -2.56800000 \\
\hline $\mathrm{H}$ & -5.44400000 & -6.67500000 & -3.45100000 \\
\hline $\mathrm{H}$ & -6.76000000 & -7.37900000 & $\begin{array}{r}-2.91100000 \\
\text { S31 }\end{array}$ \\
\hline
\end{tabular}




$\begin{array}{lrrr}\mathrm{C} & -5.13700000 & -8.04500000 & -2.00200000 \\ \mathrm{H} & -5.67900000 & -8.40700000 & -1.11700000 \\ \mathrm{C} & -5.05000000 & -9.23200000 & -2.97500000 \\ \mathrm{O} & -4.05800000 & -10.00000000 & -2.83000000 \\ \mathrm{O} & -5.93200000 & -9.32300000 & -3.85900000 \\ \mathrm{Fe} & -0.47000000 & 1.75500000 & 5.39500000 \\ \mathrm{O} & -0.57300000 & 1.41800000 & 3.21500000 \\ \mathrm{C} & -3.10300000 & 2.66300000 & 4.19500000 \\ \mathrm{O} & -3.04800000 & 1.68500000 & 3.37700000 \\ \mathrm{O} & -2.25000000 & 2.90100000 & 5.09700000 \\ \mathrm{C} & -4.28000000 & 3.61900000 & 4.08600000 \\ \mathrm{H} & -5.15300000 & 3.12500000 & 4.53100000 \\ \mathrm{H} & -4.05200000 & 4.49600000 & 4.69600000 \\ \mathrm{C} & -4.61400000 & 4.01300000 & 2.64400000 \\ \mathrm{H} & -4.63200000 & 3.09400000 & 2.05200000 \\ \mathrm{H} & -3.84500000 & 4.67300000 & 2.23400000 \\ \mathrm{C} & -5.98300000 & 4.67200000 & 2.49900000 \\ \mathrm{O} & -7.00500000 & 4.00900000 & 2.86300000 \\ \mathrm{O} & -6.02800000 & 5.83300000 & 1.99400000\end{array}$

Coordinate of ${ }^{5} \mathrm{TSa}^{\prime}$-reb

$\begin{array}{rrrr}\mathrm{C} & 0.13200000 & 1.51200000 & 10.78100000 \\ \mathrm{H} & 0.76500000 & 0.62200000 & 10.78800000 \\ \mathrm{H} & -0.67200000 & 1.34800000 & 11.50500000 \\ \mathrm{~N} & -1.53500000 & 2.49800000 & 9.12200000 \\ \mathrm{H} & -2.16900000 & 2.90000000 & 9.81700000 \\ \mathrm{C} & -0.39700000 & 1.75700000 & 9.40300000 \\ \mathrm{C} & -1.64100000 & 2.62000000 & 7.78100000 \\ \mathrm{H} & -2.44600000 & 3.11600000 & 7.26400000 \\ \mathrm{~N} & -0.63500000 & 1.99600000 & 7.18700000 \\ \mathrm{C} & 0.14600000 & 1.45400000 & 8.18100000 \\ \mathrm{H} & 1.04000000 & 0.89700000 & 7.94500000 \\ \mathrm{C} & 3.84100000 & -0.19900000 & 4.88900000 \\ \mathrm{H} & 4.60000000 & 0.50400000 & 4.52400000 \\ \mathrm{H} & 3.95100000 & -1.09400000 & 4.26700000 \\ \mathrm{C} & 2.45600000 & 0.37400000 & 4.52500000 \\ \mathrm{O} & 1.67400000 & 0.85200000 & 5.40800000 \\ \mathrm{O} & 2.21900000 & 0.31600000 & 3.28600000 \\ \mathrm{C} & 0.60000000 & 7.09200000 & 6.67000000 \\ \mathrm{H} & -0.29000000 & 7.47400000 & 6.16300000 \\ \mathrm{H} & 1.43300000 & 7.73800000 & 6.36800000 \\ \mathrm{~N} & 2.06900000 & 5.01300000 & 6.36300000 \\ \mathrm{H} & 2.93700000 & 5.34600000 & 6.79300000 \\ \mathrm{C} & 0.85300000 & 5.68000000 & 6.24300000 \\ \mathrm{C} & 1.92800000 & 3.78800000 & 5.81700000 \\ & & & 532\end{array}$




\begin{tabular}{|c|c|c|c|}
\hline $\mathrm{H}$ & 2.69800000 & 3.03400000 & 5.81900000 \\
\hline $\mathrm{N}$ & 0.70600000 & 3.62100000 & 5.33100000 \\
\hline $\mathrm{C}$ & 0.03200000 & 4.79900000 & 5.59000000 \\
\hline $\mathrm{H}$ & -0.99500000 & 4.91800000 & 5.28900000 \\
\hline $\mathrm{C}$ & -4.05400000 & -1.78200000 & 2.08000000 \\
\hline $\mathrm{O}$ & -4.45300000 & -0.88500000 & 1.35100000 \\
\hline $\mathrm{N}$ & -3.09300000 & -1.83600000 & 3.04000000 \\
\hline $\mathrm{C}$ & -2.06800000 & -0.94500000 & 3.54400000 \\
\hline $\mathrm{H}$ & -2.18400000 & 0.00900000 & 3.03700000 \\
\hline $\mathrm{C}$ & -2.31800000 & -0.71000000 & 5.04500000 \\
\hline $\mathrm{O}$ & -3.30100000 & -1.27900000 & 5.56200000 \\
\hline $\mathrm{O}$ & -1.48100000 & -0.00800000 & 5.70700000 \\
\hline $\mathrm{C}$ & -0.70900000 & -1.56400000 & 3.26600000 \\
\hline $\mathrm{C}$ & -0.72200000 & -2.50700000 & 2.11500000 \\
\hline $\mathrm{H}$ & 0.59000000 & 0.64600000 & 3.26000000 \\
\hline $\mathrm{H}$ & -1.23100000 & -2.12200000 & 1.22900000 \\
\hline $\mathrm{H}$ & 0.26500000 & -2.87900000 & 1.84100000 \\
\hline $\mathrm{C}$ & 0.17800000 & -1.99700000 & 4.40200000 \\
\hline $\mathrm{H}$ & -0.36300000 & -2.71600000 & 5.03600000 \\
\hline $\mathrm{H}$ & 1.06100000 & -2.52100000 & 4.03300000 \\
\hline $\mathrm{H}$ & 0.47800000 & -1.17800000 & 5.04900000 \\
\hline S & -1.64500000 & -4.10200000 & 2.64800000 \\
\hline $\mathrm{C}$ & -3.16800000 & -3.26300000 & 3.27100000 \\
\hline $\mathrm{H}$ & -3.34300000 & -3.53800000 & 4.31200000 \\
\hline $\mathrm{C}$ & -4.42300000 & -3.26300000 & 2.33600000 \\
\hline $\mathrm{H}$ & -5.33400000 & -3.35200000 & 2.93100000 \\
\hline $\mathrm{N}$ & -4.49000000 & -4.20200000 & 1.26000000 \\
\hline $\mathrm{H}$ & -3.84100000 & -4.10300000 & 0.49100000 \\
\hline $\mathrm{C}$ & -4.88600000 & -5.47800000 & 1.59200000 \\
\hline $\mathrm{O}$ & -5.37300000 & -5.69200000 & 2.70300000 \\
\hline $\mathrm{C}$ & -4.78700000 & -6.54200000 & 0.53700000 \\
\hline $\mathrm{H}$ & -5.75800000 & -6.54600000 & 0.02700000 \\
\hline $\mathrm{H}$ & -4.70300000 & -7.49600000 & 1.06700000 \\
\hline $\mathrm{C}$ & -3.71200000 & -6.40000000 & -0.54000000 \\
\hline $\mathrm{H}$ & -3.91000000 & -5.49800000 & -1.13200000 \\
\hline $\mathrm{H}$ & -2.72100000 & -6.28700000 & -0.08400000 \\
\hline $\mathrm{C}$ & -3.71200000 & -7.61400000 & -1.47900000 \\
\hline $\mathrm{H}$ & -3.10000000 & -7.39700000 & -2.36500000 \\
\hline $\mathrm{H}$ & -3.23000000 & -8.46200000 & -0.98800000 \\
\hline $\mathrm{N}$ & -5.87000000 & -6.91500000 & -2.46200000 \\
\hline $\mathrm{H}$ & -5.42000000 & -6.65800000 & -3.35200000 \\
\hline $\mathrm{H}$ & -6.74000000 & -7.33700000 & -2.78800000 \\
\hline $\mathrm{C}$ & -5.11200000 & -8.05000000 & -1.92700000 \\
\hline $\mathrm{H}$ & -5.65900000 & -8.43000000 & -1.05100000 \\
\hline $\mathrm{C}$ & -5.04300000 & -9.21200000 & $\begin{array}{c}-2.93500000 \\
\text { S33 }\end{array}$ \\
\hline
\end{tabular}




$\begin{array}{lllc}\mathrm{O} & -4.05600000 & -9.99100000 & -2.82700000 \\ \mathrm{O} & -5.93900000 & -9.27300000 & -3.80800000 \\ \mathrm{Fe} & -0.42900000 & 1.61400000 & 5.17500000 \\ \mathrm{O} & -0.32200000 & 1.01100000 & 3.35900000 \\ \mathrm{C} & -3.10600000 & 2.61800000 & 4.22300000 \\ \mathrm{O} & -3.42500000 & 1.55600000 & 3.67000000 \\ \mathrm{O} & -2.01300000 & 2.84100000 & 4.87700000 \\ \mathrm{C} & -4.09900000 & 3.77900000 & 4.15300000 \\ \mathrm{H} & -4.99200000 & 3.43800000 & 4.69200000 \\ \mathrm{H} & -3.70400000 & 4.66300000 & 4.66000000 \\ \mathrm{C} & -4.50900000 & 4.09200000 & 2.70100000 \\ \mathrm{H} & -4.53200000 & 3.14000000 & 2.16600000 \\ \mathrm{H} & -3.78000000 & 4.75200000 & 2.22100000 \\ \mathrm{C} & -5.90400000 & 4.70400000 & 2.55700000 \\ \mathrm{O} & -6.89400000 & 4.02000000 & 2.96000000 \\ \mathrm{O} & -5.99500000 & 5.84100000 & 2.00700000\end{array}$

Coordinate of ${ }^{5} \mathrm{~Pa}^{\prime}$-reb

$\begin{array}{lrrr}\mathrm{C} & 0.20100000 & 1.55700000 & 11.16300000 \\ \mathrm{H} & 0.85100000 & 0.68100000 & 11.18100000 \\ \mathrm{H} & -0.53400000 & 1.43100000 & 11.96300000 \\ \mathrm{~N} & -1.60200000 & 2.39000000 & 9.56000000 \\ \mathrm{H} & -2.22200000 & 2.80900000 & 10.26300000 \\ \mathrm{C} & -0.43300000 & 1.69400000 & 9.81600000 \\ \mathrm{C} & -1.76400000 & 2.45100000 & 8.21800000 \\ \mathrm{H} & -2.60200000 & 2.92200000 & 7.72800000 \\ \mathrm{~N} & -0.77200000 & 1.84000000 & 7.59400000 \\ \mathrm{C} & 0.05200000 & 1.35000000 & 8.58000000 \\ \mathrm{H} & 0.96000000 & 0.81100000 & 8.35200000 \\ \mathrm{C} & 3.75400000 & -0.16600000 & 4.83800000 \\ \mathrm{H} & 4.54400000 & 0.49100000 & 4.45300000 \\ \mathrm{H} & 3.82100000 & -1.07400000 & 4.22900000 \\ \mathrm{C} & 2.39300000 & 0.46600000 & 4.48000000 \\ \mathrm{O} & 1.58600000 & 0.86400000 & 5.35300000 \\ \mathrm{O} & 2.19800000 & 0.51600000 & 3.21300000 \\ \mathrm{C} & 0.61800000 & 7.17400000 & 6.76900000 \\ \mathrm{H} & -0.26500000 & 7.56100000 & 6.25400000 \\ \mathrm{H} & 1.47000000 & 7.78800000 & 6.45500000 \\ \mathrm{~N} & 2.03100000 & 5.04600000 & 6.45800000 \\ \mathrm{H} & 2.93500000 & 5.37600000 & 6.80800000 \\ \mathrm{C} & 0.82700000 & 5.74300000 & 6.40300000 \\ \mathrm{C} & 1.79800000 & 3.78800000 & 6.02300000 \\ \mathrm{H} & 2.53000000 & 2.99800000 & 5.99100000 \\ \mathrm{~N} & 0.53200000 & 3.64200000 & 5.68200000 \\ \mathrm{C} & -0.08200000 & 4.85000000 & 5.91300000 \\ & & & 534\end{array}$




\begin{tabular}{|c|c|c|c|}
\hline & -1.13 & $4.9^{\prime}$ & 00 \\
\hline & -4.25100000 & -1.96200000 & 2.05200000 \\
\hline & -4.64800000 & -1.12500000 & 0000 \\
\hline & -3.27200000 & -1.94100000 & 2.99200000 \\
\hline & -2.24000000 & -1.01400000 & 3.38700000 \\
\hline & -2.35800000 & -0.11800000 & 2.77100000 \\
\hline & -2.47800000 & -0.58900000 & 4.84800000 \\
\hline & -3.53100000 & -0.97100000 & 0000 \\
\hline & -1.56700000 & 0.11600000 & 5.37600000 \\
\hline & 000 & -1.60400000 & 3.08600000 \\
\hline & -0.95100000 & -2.82000000 & 2.14000000 \\
\hline & 0.71100000 & -0.36700000 & 2.74600000 \\
\hline & -1.44300000 & -2.51400000 & 1.213 \\
\hline & 0.03700000 & 500000 & 0000 \\
\hline & 0.01000000 & -2.01000000 & 4.32100000 \\
\hline $\mathrm{H}$ & -0.50100000 & -2.78700000 & 4.89600000 \\
\hline & 0.96700000 & -2.42500000 & 0000 \\
\hline & 0.16200000 & -1.15800000 & 0000 \\
\hline & -1.84900000 & -4.25300000 & 2.88800000 \\
\hline $\mathrm{C}$ & -3.37000000 & -3.33400000 & 3.37600000 \\
\hline $\mathrm{H}$ & -3.58000000 & -3.49 & 000 \\
\hline $\mathrm{C}$ & -4.61 & -3.4 & 000 \\
\hline $\mathrm{H}$ & -5.54200000 & -3.51800000 & 2.99000000 \\
\hline & -4.61800000 & -4.40500000 & 1.39000000 \\
\hline $\mathrm{H}$ & -3.93 & -4.3 & 0000 \\
\hline$C$ & -5.09900000 & -5.65400000 & 1.68700000 \\
\hline $\mathrm{O}$ & -5.68500000 & -5.85400000 & 2.75200000 \\
\hline $\mathrm{C}$ & -4.94800000 & -6.70500000 & 0.62600000 \\
\hline & -5.894 & 0000 & .07300000 \\
\hline 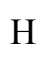 & -4.879 & -7.671 & 0000 \\
\hline $\mathrm{C}$ & -3.82800000 & -6.52600000 & -0.39900000 \\
\hline $\mathrm{H}$ & -4.00200000 & -5.60600000 & -0.97300000 \\
\hline & -2.85 & -6.4 & 000 \\
\hline $\mathrm{C}$ & -3.77700000 & -7.70400000 & -1.37600000 \\
\hline $\mathrm{H}$ & -3.11700000 & -7.46100000 & -2.21900000 \\
\hline 11 & -3.32400000 & -8.56900000 & -0.89000000 \\
\hline & -5.86600000 & -6.94400000 & -2.45200000 \\
\hline $\mathrm{H}$ & -5.37800000 & -6.68000000 & -3.31900000 \\
\hline $\mathrm{H}$ & -6.73100000 & -7.33900000 & -2.82200000 \\
\hline $\mathrm{C}$ & -5.15600000 & -8.10700000 & -1.90800000 \\
\hline $\mathrm{H}$ & -5.75400000 & -8.49700000 & -1.07200000 \\
\hline $\mathrm{C}$ & -5.05500000 & -9.23900000 & -2.94200000 \\
\hline $\mathrm{O}$ & -4.05400000 & -10.00100000 & -2.84000000 \\
\hline $\mathrm{O}$ & -5.94000000 & -9.29300000 & -3.82700000 \\
\hline 10 & -0.58400000 & 1.84400000 & $\begin{array}{c}5.38100000 \\
\text { S35 }\end{array}$ \\
\hline
\end{tabular}




$\begin{array}{lrrr}\mathrm{O} & -0.15400000 & -0.57400000 & 2.33800000 \\ \mathrm{C} & -2.99600000 & 2.95300000 & 4.21000000 \\ \mathrm{O} & -2.89400000 & 2.00400000 & 3.39000000 \\ \mathrm{O} & -2.25300000 & 3.10100000 & 5.23500000 \\ \mathrm{C} & -4.11200000 & 3.96200000 & 4.02100000 \\ \mathrm{H} & -5.00500000 & 3.50600000 & 4.46900000 \\ \mathrm{H} & -3.88600000 & 4.84500000 & 4.62700000 \\ \mathrm{C} & -4.44200000 & 4.31500000 & 2.56500000 \\ \mathrm{H} & -4.39400000 & 3.39700000 & 1.96900000 \\ \mathrm{H} & -3.71400000 & 5.02700000 & 2.16400000 \\ \mathrm{C} & -5.85500000 & 4.87700000 & 2.42300000 \\ \mathrm{O} & -6.82100000 & 4.17200000 & 2.84700000 \\ \mathrm{O} & -5.98900000 & 6.01600000 & 1.88500000\end{array}$

Coordinate of ${ }^{3} \mathrm{Ra}^{\prime}$

$\begin{array}{rrrr}\mathrm{C} & 0.21300000 & 1.46300000 & 10.84200000 \\ \mathrm{H} & 0.86100000 & 0.58500000 & 10.85900000 \\ \mathrm{H} & -0.57000000 & 1.30800000 & 11.59000000 \\ \mathrm{~N} & -1.49100000 & 2.40300000 & 9.20300000 \\ \mathrm{H} & -2.12300000 & 2.80000000 & 9.90000000 \\ \mathrm{C} & -0.34900000 & 1.66500000 & 9.46900000 \\ \mathrm{C} & -1.60300000 & 2.53000000 & 7.86100000 \\ \mathrm{H} & -2.41500000 & 3.03000000 & 7.36200000 \\ \mathrm{~N} & -0.60600000 & 1.91100000 & 7.25000000 \\ \mathrm{C} & 0.18000000 & 1.36700000 & 8.23800000 \\ \mathrm{H} & 1.06900000 & 0.80700000 & 7.99300000 \\ \mathrm{C} & 3.70300000 & -0.19500000 & 4.86100000 \\ \mathrm{H} & 4.43700000 & 0.52600000 & 4.47900000 \\ \mathrm{H} & 3.82200000 & -1.08500000 & 4.23600000 \\ \mathrm{C} & 2.29300000 & 0.34900000 & 4.52800000 \\ \mathrm{O} & 1.54200000 & 0.84200000 & 5.43100000 \\ \mathrm{O} & 2.02000000 & 0.26000000 & 3.29800000 \\ \mathrm{C} & 0.51200000 & 7.04400000 & 6.60000000 \\ \mathrm{H} & -0.38900000 & 7.43900000 & 6.12200000 \\ \mathrm{H} & 1.34500000 & 7.66800000 & 6.25900000 \\ \mathrm{~N} & 1.94800000 & 4.96500000 & 6.22400000 \\ \mathrm{H} & 2.83900000 & 5.31900000 & 6.58600000 \\ \mathrm{C} & 0.72200000 & 5.62100000 & 6.18700000 \\ \mathrm{C} & 1.77100000 & 3.71700000 & 5.74700000 \\ \mathrm{H} & 2.54900000 & 2.97800000 & 5.69900000 \\ \mathrm{~N} & 0.50900000 & 3.53000000 & 5.39100000 \\ \mathrm{C} & -0.15300000 & 4.71200000 & 5.65300000 \\ \mathrm{H} & -1.19900000 & 4.81600000 & 5.42900000 \\ \mathrm{C} & -4.09400000 & -1.72600000 & 2.03400000 \\ \mathrm{O} & -4.35500000 & -0.89800000 & 1.17400000 \\ & & & 536\end{array}$




\begin{tabular}{|c|c|c|c|}
\hline $\mathrm{N}$ & -3.29000000 & -1.70100000 & 3.13 \\
\hline $\mathrm{C}$ & -2.03100000 & -1.09800000 & 3.51900000 \\
\hline $\mathrm{H}$ & -1.84900000 & -0.18800000 & 2.95300000 \\
\hline $\mathrm{C}$ & -2.09700000 & -0.73100000 & 5.01100000 \\
\hline $\mathrm{O}$ & -3.01000000 & -1.24800000 & 5.68500000 \\
\hline $\mathrm{O}$ & -1.18500000 & 0.00500000 & 5.51000000 \\
\hline $\mathrm{C}$ & -0.95300000 & -2.18600000 & 3.15700000 \\
\hline $\mathrm{C}$ & -0.49000000 & -1.90700000 & 1.72400000 \\
\hline $\mathrm{H}$ & 0.08400000 & -0.97200000 & 1.71000000 \\
\hline $\mathrm{H}$ & -1.33400000 & -1.79700000 & 1.03700000 \\
\hline $\mathrm{H}$ & 0.12100000 & -2.73400000 & 1.35200000 \\
\hline $\mathrm{C}$ & 0.23200000 & -2.26300000 & 4.11800000 \\
\hline $\mathrm{H}$ & -0.09500000 & -2.52700000 & 5.12300000 \\
\hline $\mathrm{H}$ & 0.92800000 & -3.03400000 & 3.77500000 \\
\hline $\mathrm{H}$ & 0.75100000 & -1.30600000 & 4.16900000 \\
\hline S & -1.84100000 & -3.86600000 & 3.23000000 \\
\hline $\mathrm{C}$ & -3.48600000 & -3.10000000 & 3.48800000 \\
\hline $\mathrm{H}$ & -3.84100000 & -3.26400000 & 4.50300000 \\
\hline $\mathrm{C}$ & -4.56100000 & -3.16800000 & 2.36400000 \\
\hline $\mathrm{H}$ & -5.55900000 & -3.19600000 & 2.80500000 \\
\hline $\mathrm{N}$ & -4.47700000 & -4.18700000 & 1.35700000 \\
\hline $\mathrm{H}$ & -3.82100000 & -4.08900000 & 0.59600000 \\
\hline $\mathrm{C}$ & -4.90000000 & -5.44300000 & 1.72000000 \\
\hline $\mathrm{O}$ & -5.43000000 & -5.59700000 & 2.82200000 \\
\hline $\mathrm{C}$ & -4.79600000 & -6.55900000 & 0.71400000 \\
\hline $\mathrm{H}$ & -5.76000000 & -6.57200000 & 0.19300000 \\
\hline $\mathrm{H}$ & -4.72900000 & -7.49000000 & 1.28500000 \\
\hline $\mathrm{C}$ & -3.70900000 & -6.47100000 & -0.35600000 \\
\hline $\mathrm{H}$ & -3.87900000 & -5.57000000 & -0.96000000 \\
\hline $\mathrm{H}$ & -2.72200000 & -6.38400000 & 0.11300000 \\
\hline $\mathrm{C}$ & -3.71300000 & -7.68800000 & -1.29200000 \\
\hline $\mathrm{H}$ & -3.05400000 & -7.49000000 & -2.14600000 \\
\hline $\mathrm{H}$ & -3.27600000 & -8.54800000 & -0.77900000 \\
\hline $\mathrm{N}$ & -5.81100000 & -6.89200000 & -2.31800000 \\
\hline $\mathrm{H}$ & -5.35500000 & -6.64400000 & -3.20700000 \\
\hline $\mathrm{H}$ & -6.70200000 & -7.26800000 & -2.64400000 \\
\hline $\mathrm{C}$ & -5.10600000 & -8.07200000 & -1.80500000 \\
\hline $\mathrm{H}$ & -5.69500000 & -8.46600000 & -0.96400000 \\
\hline $\mathrm{C}$ & -5.04300000 & -9.18900000 & -2.86900000 \\
\hline $\mathrm{O}$ & -4.05300000 & -9.96900000 & -2.81600000 \\
\hline $\mathrm{O}$ & -5.95100000 & -9.20900000 & -3.73200000 \\
\hline $\mathrm{Fe}$ & -0.37400000 & 1.71600000 & 5.13000000 \\
\hline $\mathrm{O}$ & -0.31900000 & 1.58300000 & 3.50600000 \\
\hline $\mathrm{C}$ & -3.06700000 & 2.48300000 & 4.28900000 \\
\hline $\mathrm{O}$ & -3.25000000 & 1.48200000 & $\begin{array}{c}3.58200000 \\
\text { S37 }\end{array}$ \\
\hline
\end{tabular}




$\begin{array}{llll}\mathrm{O} & -2.06000000 & 2.70000000 & 5.07600000 \\ \mathrm{C} & -4.11700000 & 3.59800000 & 4.26200000 \\ \mathrm{H} & -5.03500000 & 3.17500000 & 4.68700000 \\ \mathrm{H} & -3.80200000 & 4.42900000 & 4.89900000 \\ \mathrm{C} & -4.42100000 & 4.07300000 & 2.83000000 \\ \mathrm{H} & -4.34700000 & 3.19900000 & 2.17600000 \\ \mathrm{H} & -3.68500000 & 4.81100000 & 2.49800000 \\ \mathrm{C} & -5.82900000 & 4.64100000 & 2.61300000 \\ \mathrm{O} & -6.82100000 & 3.95200000 & 3.00700000 \\ \mathrm{O} & -5.92700000 & 5.74300000 & 1.99700000\end{array}$

Coordinate of ${ }^{3} \mathrm{TS} 1 \mathrm{a}^{\prime}$

$\begin{array}{rrrr}\mathrm{C} & 0.18400000 & 1.46400000 & 10.76500000 \\ \mathrm{H} & 0.81800000 & 0.57500000 & 10.77300000 \\ \mathrm{H} & -0.60900000 & 1.30800000 & 11.50300000 \\ \mathrm{~N} & -1.47200000 & 2.48300000 & 9.12500000 \\ \mathrm{H} & -2.10000000 & 2.88600000 & 9.82200000 \\ \mathrm{C} & -0.36000000 & 1.70000000 & 9.39000000 \\ \mathrm{C} & -1.57500000 & 2.62500000 & 7.78400000 \\ \mathrm{H} & -2.37100000 & 3.15100000 & 7.28500000 \\ \mathrm{~N} & -0.59800000 & 1.97500000 & 7.17400000 \\ \mathrm{C} & 0.16300000 & 1.39100000 & 8.15900000 \\ \mathrm{H} & 1.03100000 & 0.80100000 & 7.91100000 \\ \mathrm{C} & 3.79100000 & -0.14100000 & 4.83300000 \\ \mathrm{H} & 4.53100000 & 0.59200000 & 4.48800000 \\ \mathrm{H} & 3.92800000 & -1.01600000 & 4.19100000 \\ \mathrm{C} & 2.38600000 & 0.40900000 & 4.49500000 \\ \mathrm{O} & 1.63600000 & 0.90200000 & 5.39400000 \\ \mathrm{O} & 2.10500000 & 0.33000000 & 3.26400000 \\ \mathrm{C} & 0.52100000 & 6.99900000 & 6.54900000 \\ \mathrm{H} & -0.37800000 & 7.39800000 & 6.07200000 \\ \mathrm{H} & 1.35500000 & 7.62600000 & 6.21100000 \\ \mathrm{~N} & 1.96300000 & 4.93100000 & 6.10800000 \\ \mathrm{H} & 2.85400000 & 5.27800000 & 6.47300000 \\ \mathrm{C} & 0.73200000 & 5.58000000 & 6.11800000 \\ \mathrm{C} & 1.77900000 & 3.69900000 & 5.58700000 \\ \mathrm{H} & 2.56900000 & 2.97900000 & 5.47100000 \\ \mathrm{~N} & 0.51100000 & 3.51200000 & 5.25200000 \\ \mathrm{C} & -0.14700000 & 4.68300000 & 5.57300000 \\ \mathrm{H} & -1.19800000 & 4.79200000 & 5.37400000 \\ \mathrm{C} & -4.23100000 & -1.84700000 & 2.26000000 \\ \mathrm{O} & -4.60700000 & -0.98200000 & 1.49000000 \\ \mathrm{~N} & -3.39400000 & -1.82100000 & 3.35100000 \\ \mathrm{C} & -2.25800000 & -1.00900000 & 3.68900000 \\ \mathrm{H} & -2.35700000 & -0.04600000 & 3.19700000 \\ & & & \\ & & \end{array}$




\begin{tabular}{|c|c|c|c|}
\hline $\mathrm{C}$ & -2.24400000 & -0.72700000 & 5.18800000 \\
\hline $\mathrm{O}$ & -3.10300000 & -1.28700000 & 5.89400000 \\
\hline $\mathrm{O}$ & -1.32400000 & 0.03000000 & 5.64000000 \\
\hline $\mathrm{C}$ & -0.95000000 & -1.74800000 & 3.19300000 \\
\hline $\mathrm{C}$ & -0.37100000 & -0.82500000 & 2.15400000 \\
\hline $\mathrm{H}$ & -0.27300000 & 0.35300000 & 2.83700000 \\
\hline $\mathrm{H}$ & -1.05900000 & -0.55900000 & 1.34900000 \\
\hline $\mathrm{H}$ & 0.63400000 & -1.02500000 & 1.79700000 \\
\hline $\mathrm{C}$ & 0.08900000 & -2.12200000 & 4.25800000 \\
\hline $\mathrm{H}$ & -0.36500000 & -2.74600000 & 5.03400000 \\
\hline $\mathrm{H}$ & 0.88300000 & -2.70600000 & 3.78500000 \\
\hline $\mathrm{H}$ & 0.51900000 & -1.24300000 & 4.73400000 \\
\hline S & -1.56500000 & -3.43100000 & 2.51500000 \\
\hline $\mathrm{C}$ & -3.19000000 & -3.25300000 & 3.36900000 \\
\hline $\mathrm{H}$ & -3.20400000 & -3.72600000 & 4.35200000 \\
\hline $\mathrm{C}$ & -4.43600000 & -3.37500000 & 2.45300000 \\
\hline $\mathrm{H}$ & -5.32000000 & -3.57200000 & 3.06300000 \\
\hline $\mathrm{N}$ & -4.44400000 & -4.30700000 & 1.37300000 \\
\hline $\mathrm{H}$ & -3.78800000 & -4.18500000 & 0.61500000 \\
\hline $\mathrm{C}$ & -4.91400000 & -5.57200000 & 1.65200000 \\
\hline $\mathrm{O}$ & -5.47700000 & -5.79000000 & 2.72500000 \\
\hline $\mathrm{C}$ & -4.79200000 & -6.62100000 & 0.58800000 \\
\hline $\mathrm{H}$ & -5.75300000 & -6.61100000 & 0.05800000 \\
\hline $\mathrm{H}$ & -4.72500000 & -7.58200000 & 1.10700000 \\
\hline $\mathrm{C}$ & -3.69600000 & -6.46900000 & -0.46400000 \\
\hline $\mathrm{H}$ & -3.86800000 & -5.54600000 & -1.03300000 \\
\hline $\mathrm{H}$ & -2.71000000 & -6.39000000 & 0.01300000 \\
\hline $\mathrm{C}$ & -3.69500000 & -7.65400000 & -1.43800000 \\
\hline $\mathrm{H}$ & -3.08600000 & -7.40900000 & -2.31800000 \\
\hline $\mathrm{H}$ & -3.20900000 & -8.51400000 & -0.97300000 \\
\hline $\mathrm{N}$ & -5.84800000 & -6.92300000 & -2.40000000 \\
\hline $\mathrm{H}$ & -5.41600000 & -6.66900000 & -3.29900000 \\
\hline $\mathrm{H}$ & -6.73600000 & -7.32300000 & -2.70100000 \\
\hline $\mathrm{C}$ & -5.09700000 & -8.07500000 & -1.89300000 \\
\hline $\mathrm{H}$ & -5.64400000 & -8.47100000 & -1.02500000 \\
\hline $\mathrm{C}$ & -5.03900000 & -9.21500000 & -2.92500000 \\
\hline $\mathrm{O}$ & -4.05400000 & -9.99900000 & -2.83300000 \\
\hline $\mathrm{O}$ & -5.94000000 & -9.25700000 & -3.79300000 \\
\hline $\mathrm{Fe}$ & -0.43300000 & 1.66300000 & 5.10200000 \\
\hline $\mathrm{O}$ & -0.47900000 & 1.43200000 & 3.36000000 \\
\hline $\mathrm{C}$ & -3.15900000 & 2.55500000 & 4.31000000 \\
\hline $\mathrm{O}$ & -3.49000000 & 1.53500000 & 3.68600000 \\
\hline $\mathrm{O}$ & -2.07600000 & 2.73800000 & 4.99100000 \\
\hline $\mathrm{C}$ & -4.12700000 & 3.73600000 & 4.28300000 \\
\hline $\mathrm{H}$ & -5.05900000 & 3.37200000 & $\begin{array}{c}4.73100000 \\
\text { S39 }\end{array}$ \\
\hline
\end{tabular}




$\begin{array}{llll}\mathrm{H} & -3.75300000 & 4.56400000 & 4.89200000 \\ \mathrm{C} & -4.43600000 & 4.19300000 & 2.84500000 \\ \mathrm{H} & -4.34600000 & 3.31700000 & 2.19600000 \\ \mathrm{H} & -3.71900000 & 4.94500000 & 2.50700000 \\ \mathrm{C} & -5.85800000 & 4.71900000 & 2.62500000 \\ \mathrm{O} & -6.83100000 & 4.01300000 & 3.03200000 \\ \mathrm{O} & -5.98300000 & 5.81200000 & 1.99700000\end{array}$

Coordinate of ${ }^{3} \mathrm{IM} 1 \mathrm{a}^{\prime}$

$\begin{array}{lrrr}\mathrm{C} & 0.13600000 & 1.47600000 & 10.76400000 \\ \mathrm{H} & 0.78100000 & 0.59600000 & 10.76500000 \\ \mathrm{H} & -0.65400000 & 1.30000000 & 11.50100000 \\ \mathrm{~N} & -1.55300000 & 2.45000000 & 9.12400000 \\ \mathrm{H} & -2.17700000 & 2.85600000 & 9.82300000 \\ \mathrm{C} & -0.41500000 & 1.70300000 & 9.39100000 \\ \mathrm{C} & -1.68700000 & 2.55100000 & 7.78300000 \\ \mathrm{H} & -2.49400000 & 3.05700000 & 7.27900000 \\ \mathrm{~N} & -0.70400000 & 1.90300000 & 7.17500000 \\ \mathrm{C} & 0.09500000 & 1.37300000 & 8.16100000 \\ \mathrm{H} & 0.97900000 & 0.80600000 & 7.91500000 \\ \mathrm{C} & 3.79100000 & -0.14100000 & 4.83300000 \\ \mathrm{H} & 4.53100000 & 0.59200000 & 4.48800000 \\ \mathrm{H} & 3.92800000 & -1.01600000 & 4.19100000 \\ \mathrm{C} & 2.38600000 & 0.40900000 & 4.49500000 \\ \mathrm{O} & 1.63600000 & 0.90200000 & 5.39400000 \\ \mathrm{O} & 2.10500000 & 0.33000000 & 3.26400000 \\ \mathrm{C} & 0.58000000 & 7.19000000 & 6.72100000 \\ \mathrm{H} & -0.30500000 & 7.60300000 & 6.22800000 \\ \mathrm{H} & 1.42900000 & 7.81700000 & 6.42600000 \\ \mathrm{~N} & 2.02300000 & 5.12200000 & 6.31000000 \\ \mathrm{H} & 0.55200000 & 1.03300000 & 3.22300000 \\ \mathrm{C} & 0.79900000 & 5.77500000 & 6.29100000 \\ \mathrm{C} & 1.83400000 & 3.87300000 & 5.83200000 \\ \mathrm{H} & 2.61400000 & 3.13200000 & 5.76600000 \\ \mathrm{H} & 0.57400000 & 3.68100000 & 5.49300000 \\ \mathrm{C} & -0.08000000 & 4.86200000 & 5.77300000 \\ \mathrm{H} & -1.13700000 & 4.96800000 & 5.59000000 \\ \mathrm{O} & -4.13900000 & -1.82300000 & 2.04600000 \\ \mathrm{~N} & -4.37200000 & -1.02000000 & 1.15700000 \\ \mathrm{H} & -3.33700000 & -1.79300000 & 3.15100000 \\ \mathrm{H} & -1.87800000 & -0.29200000 & 2.96900000 \\ \mathrm{H} & -10900000 & -0.84500000 & 5.02500000 \\ \mathrm{H} & -13400000 & -0.16000000 & 5.47700000 \\ \mathrm{H} & & 540\end{array}$




\begin{tabular}{|c|c|c|c|}
\hline $\mathrm{C}$ & -1.00100000 & -2.30000000 & 3.16500000 \\
\hline $\mathrm{C}$ & -0.61700000 & -2.16100000 & 1.75100000 \\
\hline $\mathrm{H}$ & 0.57600000 & 1.15300000 & 3.19000000 \\
\hline $\mathrm{H}$ & -1.27200000 & -1.65600000 & 1.04800000 \\
\hline $\mathrm{H}$ & 0.15000000 & -2.81100000 & 1.34100000 \\
\hline $\mathrm{C}$ & 0.20000000 & -2.41600000 & 4.10300000 \\
\hline $\mathrm{H}$ & -0.11200000 & -2.64800000 & 5.12000000 \\
\hline $\mathrm{H}$ & 0.85300000 & -3.22000000 & 3.75000000 \\
\hline $\mathrm{H}$ & 0.76200000 & -1.48100000 & 4.12500000 \\
\hline S & -1.95300000 & -4.00900000 & 3.32400000 \\
\hline $\mathrm{C}$ & -3.57100000 & -3.17900000 & 3.53200000 \\
\hline $\mathrm{H}$ & -3.93700000 & -3.30800000 & 4.54900000 \\
\hline $\mathrm{C}$ & -4.63600000 & -3.24700000 & 2.39800000 \\
\hline $\mathrm{H}$ & -5.63900000 & -3.26000000 & 2.82700000 \\
\hline $\mathrm{N}$ & -4.54600000 & -4.26600000 & 1.39600000 \\
\hline $\mathrm{H}$ & -3.87400000 & -4.17600000 & 0.64900000 \\
\hline $\mathrm{C}$ & -5.00400000 & -5.51600000 & 1.73500000 \\
\hline $\mathrm{O}$ & -5.57300000 & -5.67300000 & 2.81700000 \\
\hline $\mathrm{C}$ & -4.87900000 & -6.61900000 & 0.71900000 \\
\hline $\mathrm{H}$ & -5.83500000 & -6.63000000 & 0.18100000 \\
\hline $\mathrm{H}$ & -4.81800000 & -7.55800000 & 1.27900000 \\
\hline $\mathrm{C}$ & -3.77400000 & -6.51000000 & -0.32900000 \\
\hline $\mathrm{H}$ & -3.94400000 & -5.60600000 & -0.92900000 \\
\hline $\mathrm{H}$ & -2.79700000 & -6.41500000 & 0.15900000 \\
\hline $\mathrm{C}$ & -3.75200000 & -7.71700000 & -1.27400000 \\
\hline $\mathrm{H}$ & -3.07100000 & -7.51400000 & -2.11000000 \\
\hline $\mathrm{H}$ & -3.33000000 & -8.58100000 & -0.75600000 \\
\hline $\mathrm{N}$ & -5.81900000 & -6.91100000 & -2.35700000 \\
\hline $\mathrm{H}$ & -5.33600000 & -6.65900000 & -3.23100000 \\
\hline $\mathrm{H}$ & -6.69800000 & -7.28500000 & -2.71400000 \\
\hline $\mathrm{C}$ & -5.13200000 & -8.09300000 & -1.82500000 \\
\hline $\mathrm{H}$ & -5.74700000 & -8.48500000 & -1.00000000 \\
\hline $\mathrm{C}$ & -5.04800000 & -9.20800000 & -2.88700000 \\
\hline $\mathrm{O}$ & -4.05100000 & -9.97800000 & -2.82200000 \\
\hline $\mathrm{O}$ & -5.94600000 & -9.23800000 & -3.75900000 \\
\hline $\mathrm{Fe}$ & -0.44300000 & 1.69100000 & 5.15400000 \\
\hline $\mathrm{O}$ & -0.28900000 & 1.58700000 & 3.36300000 \\
\hline $\mathrm{C}$ & -3.15900000 & 2.49000000 & 4.29600000 \\
\hline $\mathrm{O}$ & -3.40000000 & 1.36800000 & 3.84000000 \\
\hline $\mathrm{O}$ & -2.05900000 & 2.84100000 & 4.90200000 \\
\hline $\mathrm{C}$ & -4.21100000 & 3.58900000 & 4.18100000 \\
\hline $\mathrm{H}$ & -5.11400000 & 3.21200000 & 4.67500000 \\
\hline $\mathrm{H}$ & -3.87900000 & 4.48500000 & 4.71400000 \\
\hline $\mathrm{C}$ & -4.55700000 & 3.91700000 & 2.71900000 \\
\hline $\mathrm{H}$ & -4.59600000 & 2.97100000 & $\begin{array}{c}2.17300000 \\
\text { S41 }\end{array}$ \\
\hline
\end{tabular}




$\begin{array}{llll}\mathrm{H} & -3.78300000 & 4.54700000 & 2.27400000 \\ \mathrm{C} & -5.92700000 & 4.58100000 & 2.55500000 \\ \mathrm{O} & -6.94500000 & 3.93600000 & 2.95900000 \\ \mathrm{O} & -5.97500000 & 5.71700000 & 1.99700000\end{array}$

Coordinate of ${ }^{3} \mathrm{TS} 2 \mathrm{a}^{\prime}$

\begin{tabular}{|c|c|c|c|}
\hline & 0.13500000 & 1.47700000 & 0000 \\
\hline $\mathrm{H}$ & 0.77800000 & 0.59400000 & 10.75900000 \\
\hline & -0.65800000 & 1.30100000 & 11.49200000 \\
\hline & -1.55200000 & 2.45400000 & 9.11500000 \\
\hline 1 & -2.17500000 & 2.85900000 & 9.81500000 \\
\hline & -0.41400000 & 1.70600000 & 9.38300000 \\
\hline & -1.68500000 & 2.55400000 & 7.77400000 \\
\hline $\mathrm{H}$ & -2.49100000 & 3.06100000 & 7.27200000 \\
\hline N & -0.70200000 & 1.90700000 & 7.16700000 \\
\hline & 0.09700000 & 1.37500000 & 8.15300000 \\
\hline $\mathrm{H}$ & 0.98100000 & 0.80900000 & 7.90600000 \\
\hline $\mathrm{C}$ & 3.74800000 & -0.13200000 & 4.78400000 \\
\hline $\mathrm{H}$ & 4.58300000 & 0.43300000 & 4.35500000 \\
\hline 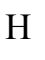 & 3.68600000 & -1.05300000 & 4.19200000 \\
\hline C & 2.45600000 & 0.64800000 & 4.46000000 \\
\hline $\mathrm{O}$ & 1.59400000 & 0.90000000 & 5.36300000 \\
\hline $\mathrm{O}$ & 2.35600000 & 0.96200000 & 3.24100000 \\
\hline $\mathrm{C}$ & 0.57900000 & 7.18700000 & 6.71800000 \\
\hline $\mathrm{H}$ & -0.30600000 & 7.59800000 & 6.22600000 \\
\hline $\mathrm{H}$ & 1.42700000 & 7.81500000 & 6.42200000 \\
\hline $\mathrm{N}$ & 2.02400000 & 5.11900000 & 6.30600000 \\
\hline $\mathrm{H}$ & 2.91800000 & 0000 & 00000 \\
\hline $\mathrm{C}$ & 0.79900000 & 5.77300000 & 6.28600000 \\
\hline $\mathrm{C}$ & 1.83600000 & 3.87100000 & 5.82400000 \\
\hline $\mathrm{H}$ & 2.61600000 & 3.13100000 & 5.75800000 \\
\hline $\mathrm{N}$ & 0.57600000 & 3.68000000 & 5.48100000 \\
\hline $\mathrm{C}$ & -0.07800000 & 4.86000000 & 5.76400000 \\
\hline $\mathrm{H}$ & -1.13500000 & 4.96600000 & 5.57900000 \\
\hline $\mathrm{C}$ & -4.13300000 & -1.83400000 & 2.05200000 \\
\hline $\mathrm{O}$ & -4.36400000 & -1.01800000 & 1.17100000 \\
\hline $\mathrm{N}$ & -3.30700000 & -1.82900000 & 3.13600000 \\
\hline $\mathrm{C}$ & -2.07000000 & -1.19100000 & 3.52300000 \\
\hline $\mathrm{H}$ & -1.93700000 & -0.25800000 & 2.97200000 \\
\hline $\mathrm{C}$ & -2.11000000 & -0.84000000 & 5.02000000 \\
\hline $\mathrm{O}$ & -3.04200000 & -1.29900000 & 5.70300000 \\
\hline $\mathrm{O}$ & -1.13500000 & -0.15400000 & 5.47400000 \\
\hline $\mathrm{C}$ & -0.93400000 & -2.18300000 & 3.14300000 \\
\hline $\mathrm{C}$ & -0.64100000 & -2.18100000 & 1.76100000 \\
\hline $\mathrm{H}$ & 0.58300000 & 1.15600000 & 3.1870000 \\
\hline
\end{tabular}




\begin{tabular}{|c|c|c|c|}
\hline $\mathrm{H}$ & -1.31900000 & -1.72900000 & 1.04500000 \\
\hline $\mathrm{H}$ & 0.16200000 & -2.79500000 & 1.36600000 \\
\hline $\mathrm{C}$ & 0.21600000 & -2.39400000 & 4.10800000 \\
\hline $\mathrm{H}$ & -0.13700000 & -2.65700000 & 5.10200000 \\
\hline $\mathrm{H}$ & 0.85800000 & -3.20000000 & 3.74000000 \\
\hline $\mathrm{H}$ & 0.80200000 & -1.47600000 & 4.19500000 \\
\hline S & -2.02400000 & -4.14300000 & 3.34200000 \\
\hline $\mathrm{C}$ & -3.57200000 & -3.21100000 & 3.53100000 \\
\hline $\mathrm{H}$ & -3.94700000 & -3.30600000 & 4.55000000 \\
\hline $\mathrm{C}$ & -4.65000000 & -3.24900000 & 2.40300000 \\
\hline $\mathrm{H}$ & -5.65100000 & -3.25000000 & 2.83500000 \\
\hline $\mathrm{N}$ & -4.57200000 & -4.26400000 & 1.39500000 \\
\hline $\mathrm{H}$ & -3.89300000 & -4.17800000 & 0.65400000 \\
\hline $\mathrm{C}$ & -5.02100000 & -5.51600000 & 1.73600000 \\
\hline $\mathrm{O}$ & -5.59100000 & -5.67600000 & 2.81800000 \\
\hline $\mathrm{C}$ & -4.88800000 & -6.62000000 & 0.72100000 \\
\hline $\mathrm{H}$ & -5.84300000 & -6.63500000 & 0.18100000 \\
\hline $\mathrm{H}$ & -4.82500000 & -7.55800000 & 1.28200000 \\
\hline $\mathrm{C}$ & -3.78200000 & -6.50800000 & -0.32500000 \\
\hline $\mathrm{H}$ & -3.95200000 & -5.60400000 & -0.92500000 \\
\hline $\mathrm{H}$ & -2.80600000 & -6.41100000 & 0.16500000 \\
\hline $\mathrm{C}$ & -3.75600000 & -7.71500000 & -1.27100000 \\
\hline $\mathrm{H}$ & -3.07400000 & -7.51000000 & -2.10600000 \\
\hline $\mathrm{H}$ & -3.33300000 & -8.57900000 & -0.75300000 \\
\hline $\mathrm{N}$ & -5.82100000 & -6.91100000 & -2.36000000 \\
\hline $\mathrm{H}$ & -5.33700000 & -6.65900000 & -3.23300000 \\
\hline $\mathrm{H}$ & -6.69900000 & -7.28600000 & -2.71800000 \\
\hline $\mathrm{C}$ & -5.13400000 & -8.09300000 & -1.82500000 \\
\hline $\mathrm{H}$ & -5.75000000 & -8.48500000 & -1.00200000 \\
\hline $\mathrm{C}$ & -5.04800000 & -9.20800000 & -2.88700000 \\
\hline $\mathrm{O}$ & -4.05000000 & -9.97800000 & -2.82100000 \\
\hline $\mathrm{O}$ & -5.94600000 & -9.23900000 & -3.76000000 \\
\hline $\mathrm{Fe}$ & -0.44200000 & 1.69300000 & 5.14700000 \\
\hline $\mathrm{O}$ & -0.28500000 & 1.58400000 & 3.35500000 \\
\hline $\mathrm{C}$ & -3.16300000 & 2.49100000 & 4.29200000 \\
\hline $\mathrm{O}$ & -3.40900000 & 1.36700000 & 3.84200000 \\
\hline $\mathrm{O}$ & -2.05900000 & 2.84300000 & 4.89000000 \\
\hline $\mathrm{C}$ & -4.21400000 & 3.59000000 & 4.17700000 \\
\hline $\mathrm{H}$ & -5.11900000 & 3.21100000 & 4.66700000 \\
\hline $\mathrm{H}$ & -3.88400000 & 4.48400000 & 4.71400000 \\
\hline $\mathrm{C}$ & -4.55800000 & 3.92300000 & 2.71500000 \\
\hline $\mathrm{H}$ & -4.59500000 & 2.97800000 & 2.16500000 \\
\hline $\mathrm{H}$ & -3.78400000 & 4.55500000 & 2.27300000 \\
\hline $\mathrm{C}$ & -5.93000000 & 4.58400000 & 2.55300000 \\
\hline $\mathrm{O}$ & -6.94600000 & 3.93700000 & $\begin{array}{c}2.95700000 \\
\text { s43 }\end{array}$ \\
\hline
\end{tabular}




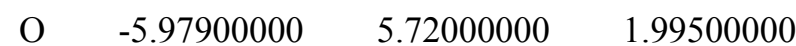

Coordinate of ${ }^{3} \mathrm{IM} 2 \mathrm{a}^{\prime}$

C $\quad 0.14100000$

$\begin{array}{llll}\mathrm{H} & 0.78000000 & 0.61300000 & 10.77600000\end{array}$

$\begin{array}{llll}\mathrm{H} & -0.65600000 & 1.33000000 & 11.50200000\end{array}$

$\begin{array}{llll}\mathrm{N} & -1.54800000 & 2.46800000 & 9.12600000\end{array}$

$\begin{array}{llll}\mathrm{H} & -2.17600000 & 2.86700000 & 9.82700000\end{array}$

$\begin{array}{llll}\text { C } & -0.40300000 & 1.73200000 & 9.39500000\end{array}$

$\begin{array}{llll}\text { C } & -1.67300000 & 2.58100000 & 7.78600000\end{array}$

$\mathrm{H} \quad-2.48300000 \quad 3.07700000 \quad 7.27800000$

$\begin{array}{llll}\mathrm{N} & -0.67700000 & 1.95300000 & 7.17900000\end{array}$

$\begin{array}{llll}\text { C } & 0.12100000 & 1.42100000 & 8.16600000\end{array}$

$\begin{array}{llll}\mathrm{H} & 1.01500000 & 0.86900000 & 7.92100000\end{array}$

$\begin{array}{llll}\text { C } & 3.79100000 & -0.14100000 & 4.83300000\end{array}$

$\mathrm{H} \quad 4.53100000 \quad 0.59200000 \quad 4.48800000$

$\begin{array}{llll}\mathrm{H} & 3.92800000 & -1.01600000 & 4.19100000\end{array}$

$\begin{array}{llll}\text { C } & 2.38600000 & 0.40900000 & 4.49500000\end{array}$

$\begin{array}{llll}\text { O } & 1.63600000 & 0.90200000 & 5.39400000\end{array}$

$\begin{array}{llll}\text { O } & 2.10500000 & 0.33000000 & 3.26400000\end{array}$

$\begin{array}{llll}\text { C } & 0.58500000 & 7.15800000 & 6.69200000\end{array}$

$\begin{array}{llll}\mathrm{H} & -0.30200000 & 7.55500000 & 6.19300000\end{array}$

$\begin{array}{llll}\mathrm{H} & 1.42600000 & 7.79600000 & 6.39500000\end{array}$

$\begin{array}{llll}\mathrm{N} & 2.03200000 & 5.07100000 & 6.37700000\end{array}$

$\begin{array}{llll}\mathrm{H} & 2.90800000 & 5.40300000 & 6.79300000\end{array}$

$\begin{array}{llll}\text { C } & 0.82000000 & 5.74400000 & 6.26200000\end{array}$

$\begin{array}{llll}\mathrm{C} & 1.86900000 & 3.83000000 & 5.87000000\end{array}$

$\mathrm{H} \quad 2.62700000 \quad 3.06500000 \quad 5.88300000$

$\begin{array}{llll}\mathrm{N} & 0.63900000 & 3.66700000 & 5.41400000\end{array}$

$\begin{array}{llll}\text { C } & -0.02300000 & 4.85300000 & 5.64900000\end{array}$

$\begin{array}{llll}\mathrm{H} & -1.05300000 & 4.97300000 & 5.35400000\end{array}$

$\begin{array}{llll}\text { C } & -4.02200000 & -1.81000000 & 2.06000000\end{array}$

$\begin{array}{llll}\mathrm{O} & -4.33900000 & -0.89000000 & 1.31900000\end{array}$

$\begin{array}{llll}\mathrm{N} & -3.03500000 & -1.95300000 & 2.98300000\end{array}$

C $\quad-1.91800000 \quad-1.16600000 \quad 3.46500000$

$\mathrm{H} \quad-1.88800000 \quad-0.22000000 \quad 2.91600000$

$\begin{array}{llll}\text { C } & -2.12600000 & -0.80200000 & 4.94700000\end{array}$

$\begin{array}{llll}\text { O } & -3.12300000 & -1.26600000 & 5.53300000\end{array}$

$\begin{array}{llll}\mathrm{O} & -1.21800000 & -0.09800000 & 5.50000000\end{array}$

$\begin{array}{llll}\text { C } & -0.64900000 & -1.98000000 & 3.21000000\end{array}$

$\begin{array}{llll}\text { C } & -0.44100000 & -2.43200000 & 1.95100000\end{array}$

$\begin{array}{llll}\mathrm{H} & 0.55200000 & 1.03300000 & 3.22300000\end{array}$

$\mathrm{H} \quad-1.08900000 \quad-2.14300000 \quad 1.13000000$

$\begin{array}{llll}\mathrm{H} & 0.41200000 & -3.05500000 & 1.71400000\end{array}$

$\begin{array}{llll}\text { C } & 0.23600000 & -2.38600000 & 4.35200000\end{array}$ 


\begin{tabular}{|c|c|c|c|}
\hline $\mathrm{H}$ & -0.36400000 & -2.88700000 & 700000 \\
\hline $\mathrm{H}$ & 1.00100000 & -3.08300000 & 4.00800000 \\
\hline $\mathrm{H}$ & 0.69800000 & -1.52300000 & 4.83100000 \\
\hline $\mathrm{S}$ & -2.04000000 & -4.52900000 & 2.94500000 \\
\hline $\mathrm{C}$ & -3.35200000 & -3.33200000 & 3.35800000 \\
\hline $\mathrm{H}$ & -3.62300000 & -3.42100000 & 4.41000000 \\
\hline $\mathrm{C}$ & -4.56600000 & -3.22300000 & 2.36700000 \\
\hline $\mathrm{H}$ & -5.50800000 & -3.20600000 & 2.91600000 \\
\hline $\mathrm{N}$ & -4.65500000 & -4.17600000 & 1.30000000 \\
\hline $\mathrm{H}$ & -3.99400000 & -4.10300000 & 0.53900000 \\
\hline $\mathrm{C}$ & -5.06900000 & -5.44400000 & 1.64600000 \\
\hline $\mathrm{O}$ & -5.56800000 & -5.63300000 & 2.75700000 \\
\hline $\mathrm{C}$ & -4.97200000 & -6.52800000 & 0.60600000 \\
\hline $\mathrm{H}$ & -5.90000000 & -6.48300000 & 0.02100000 \\
\hline $\mathrm{H}$ & -4.98000000 & -7.47600000 & 1.15100000 \\
\hline $\mathrm{C}$ & -3.81000000 & -6.46200000 & -0.38700000 \\
\hline $\mathrm{H}$ & -3.91000000 & -5.55800000 & -1.00100000 \\
\hline $\mathrm{H}$ & -2.85900000 & -6.39700000 & 0.15500000 \\
\hline $\mathrm{C}$ & -3.78300000 & -7.68500000 & -1.31600000 \\
\hline $\mathrm{H}$ & -3.09300000 & -7.49700000 & -2.14800000 \\
\hline $\mathrm{H}$ & -3.36700000 & -8.54200000 & -0.78200000 \\
\hline $\mathrm{N}$ & -5.84600000 & -6.91600000 & -2.44700000 \\
\hline $\mathrm{H}$ & -5.34100000 & -6.66300000 & -3.30700000 \\
\hline $\mathrm{H}$ & -6.70300000 & -7.31400000 & -2.83100000 \\
\hline $\mathrm{C}$ & -5.15500000 & -8.07800000 & -1.87500000 \\
\hline $\mathrm{H}$ & -5.77700000 & -8.45200000 & -1.04800000 \\
\hline $\mathrm{C}$ & -5.05200000 & -9.21700000 & -2.90900000 \\
\hline $\mathrm{O}$ & -4.04900000 & -9.97800000 & -2.82100000 \\
\hline $\mathrm{O}$ & -5.94200000 & -9.27300000 & -3.78900000 \\
\hline $\mathrm{Fe}$ & -0.43600000 & 1.71900000 & 5.16000000 \\
\hline $\mathrm{O}$ & -0.23300000 & 1.61100000 & 3.37900000 \\
\hline $\mathrm{C}$ & -3.12500000 & 2.51100000 & 4.21800000 \\
\hline $\mathrm{O}$ & -3.31900000 & 1.45300000 & 3.60100000 \\
\hline $\mathrm{O}$ & -2.09800000 & 2.78200000 & 4.96200000 \\
\hline $\mathrm{C}$ & -4.18300000 & 3.61500000 & 4.15200000 \\
\hline $\mathrm{H}$ & -5.07100000 & 3.22600000 & 4.66800000 \\
\hline $\mathrm{H}$ & -3.83500000 & 4.49800000 & 4.69200000 \\
\hline $\mathrm{C}$ & -4.57600000 & 3.96100000 & 2.70600000 \\
\hline $\mathrm{H}$ & -4.61900000 & 3.01900000 & 2.15300000 \\
\hline $\mathrm{H}$ & -3.82600000 & 4.60700000 & 2.24200000 \\
\hline $\mathrm{C}$ & -5.95100000 & 4.61700000 & 2.56600000 \\
\hline $\mathrm{O}$ & -6.96200000 & 3.96100000 & 2.96700000 \\
\hline $\mathrm{O}$ & -6.00800000 & 5.75800000 & 2.01900000 \\
\hline
\end{tabular}

Coordinate of ${ }^{3} \mathrm{TS} 3 \mathrm{a}^{\prime}$ 


\begin{tabular}{|c|c|c|c|}
\hline & 0.16100000 & 1.47900000 & 10 . \\
\hline $\mathrm{H}$ & 0.80500000 & 0.59800000 & 10.87300000 \\
\hline & -0.62500000 & 1.32800000 & 11.59700000 \\
\hline & -1.54700000 & 2.39800000 & 9.20000000 \\
\hline 1 & -2.17800000 & 2.80500000 & 9.89500000 \\
\hline & -0.39600000 & 1.67700000 & 9.47700000 \\
\hline & -1.66300000 & 2.50600000 & 0000 \\
\hline $\mathrm{H}$ & -2.47700000 & 2.99200000 & 7.34600000 \\
\hline & -0.65800000 & 1.88800000 & 7.25600000 \\
\hline & 0.13600000 & 1.36500000 & 8.25100000 \\
\hline $\mathrm{H}$ & 1.03400000 & 0.81800000 & 8.01500000 \\
\hline $\mathrm{C}$ & 3.70300000 & -0.19500000 & 4.86100000 \\
\hline $\mathrm{H}$ & 4.43700000 & 0.52600000 & 4.47900000 \\
\hline I & 3.82200000 & -1.08500000 & 00000 \\
\hline $\mathrm{C}$ & 2.29300000 & 0.34900000 & 4.52800000 \\
\hline $\mathrm{O}$ & 1.54200000 & 0.84200000 & 5.4310000 \\
\hline $\mathrm{O}$ & 2.02000000 & 0.26000000 & 3.29800000 \\
\hline $\mathrm{C}$ & 0.53600000 & 7.06600000 & 6.63800000 \\
\hline $\mathrm{H}$ & -0.35100000 & 7.47100000 & 6.14500000 \\
\hline $\mathrm{H}$ & 1.38500000 & 7.67800000 & 6.31100000 \\
\hline $\mathrm{N}$ & 1.93800000 & 4.95000000 & 6.32800000 \\
\hline $\mathrm{H}$ & 2.82600000 & 5.28900000 & 000000 \\
\hline $\mathrm{C}$ & 0.73100000 & 5.63700000 & 6.23700000 \\
\hline $\mathrm{C}$ & 1.74000000 & 3.69600000 & 5.87300000 \\
\hline $\mathrm{H}$ & 2.47800000 & 2.91300000 & 5.89200000 \\
\hline $\mathrm{N}$ & 0.48600000 & 3.54000000 & 5.47100000 \\
\hline $\mathrm{C}$ & -0.15000000 & 4.74400000 & 5.68700000 \\
\hline $\mathrm{H}$ & -1.18700000 & 4.86600000 & 5.42600000 \\
\hline $\mathrm{C}$ & -3.99300000 & -1.79500000 & 2.05900000 \\
\hline $\mathrm{O}$ & -4.31600000 & -0.87100000 & 1.32600000 \\
\hline $\mathrm{N}$ & -2.99500000 & -1.94400000 & 2.97100000 \\
\hline $\mathrm{C}$ & -1.87700000 & -1.16200000 & 3.46300000 \\
\hline $\mathrm{H}$ & -1.80000000 & -0.23600000 & 2.88500000 \\
\hline $\mathrm{C}$ & -2.12100000 & -0.73700000 & 4.92500000 \\
\hline $\mathrm{O}$ & -3.09200000 & -1.23100000 & 5.52700000 \\
\hline $\mathrm{O}$ & -1.23500000 & 0.00400000 & 5.46700000 \\
\hline$C$ & -0.63800000 & -2.04000000 & 3.30100000 \\
\hline $\mathrm{C}$ & -0.54600000 & -2.78600000 & 2.15000000 \\
\hline $\mathrm{H}$ & 0.47100000 & 1.00600000 & 3.23800000 \\
\hline $\mathrm{H}$ & -1.13800000 & -2.54600000 & 1.27400000 \\
\hline $\mathrm{H}$ & 0.32600000 & -3.40500000 & 1.98300000 \\
\hline $\mathrm{C}$ & 0.24600000 & -2.35700000 & 4.46600000 \\
\hline $\mathrm{H}$ & -0.33900000 & -2.92500000 & 5.20100000 \\
\hline $\mathrm{H}$ & 1.08500000 & -2.97900000 & 4.15700000 \\
\hline $\mathrm{H}$ & 0.59800000 & -1.46000000 & $\begin{array}{c}4.97200000 \\
546\end{array}$ \\
\hline
\end{tabular}




\begin{tabular}{|c|c|c|c|}
\hline $\mathrm{S}$ & -2.01900000 & -4.54200000 & 2.93100000 \\
\hline $\mathrm{C}$ & -3.31900000 & -3.32200000 & 3.35000000 \\
\hline $\mathrm{H}$ & -3.58700000 & -3.41000000 & 4.40200000 \\
\hline $\mathrm{C}$ & -4.53800000 & -3.20700000 & 2.36900000 \\
\hline $\mathrm{H}$ & -5.48100000 & -3.18300000 & 2.91800000 \\
\hline $\mathrm{N}$ & -4.63900000 & -4.16400000 & 1.30500000 \\
\hline $\mathrm{H}$ & -3.97500000 & -4.10400000 & 0.54600000 \\
\hline $\mathrm{C}$ & -5.06900000 & -5.42600000 & 1.65400000 \\
\hline $\mathrm{O}$ & -5.57700000 & -5.60700000 & 2.76200000 \\
\hline $\mathrm{C}$ & -4.98200000 & -6.51500000 & 0.61600000 \\
\hline $\mathrm{H}$ & -5.90800000 & -6.46300000 & 0.02900000 \\
\hline $\mathrm{H}$ & -5.00100000 & -7.46200000 & 1.16400000 \\
\hline $\mathrm{C}$ & -3.81500000 & -6.46400000 & -0.37100000 \\
\hline $\mathrm{H}$ & -3.90600000 & -5.56100000 & -0.99000000 \\
\hline $\mathrm{H}$ & -2.86800000 & -6.40200000 & 0.17700000 \\
\hline $\mathrm{C}$ & -3.78800000 & -7.69000000 & -1.29600000 \\
\hline $\mathrm{H}$ & -3.09000000 & -7.50800000 & -2.12400000 \\
\hline $\mathrm{H}$ & -3.38000000 & -8.54800000 & -0.75600000 \\
\hline $\mathrm{N}$ & -5.84200000 & -6.91300000 & -2.43900000 \\
\hline $\mathrm{H}$ & -5.33400000 & -6.66100000 & -3.29800000 \\
\hline $\mathrm{H}$ & -6.69900000 & -7.30800000 & -2.82700000 \\
\hline $\mathrm{C}$ & -5.15800000 & -8.07800000 & -1.86500000 \\
\hline $\mathrm{H}$ & -5.78700000 & -8.45100000 & -1.04300000 \\
\hline $\mathrm{C}$ & -5.05300000 & -9.21400000 & -2.90300000 \\
\hline $\mathrm{O}$ & -4.04900000 & -9.97400000 & -2.81900000 \\
\hline $\mathrm{O}$ & -5.94300000 & -9.26900000 & -3.78300000 \\
\hline $\mathrm{Fe}$ & -0.41300000 & 1.73100000 & 5.21100000 \\
\hline $\mathrm{O}$ & -0.22200000 & 1.67700000 & 3.42200000 \\
\hline $\mathrm{C}$ & -3.08300000 & 2.46100000 & 4.27800000 \\
\hline $\mathrm{O}$ & -3.27400000 & 1.41400000 & 3.64400000 \\
\hline $\mathrm{O}$ & -2.05900000 & 2.74300000 & 5.02300000 \\
\hline $\mathrm{C}$ & -4.14700000 & 3.56500000 & 4.21500000 \\
\hline $\mathrm{H}$ & -5.04000000 & 3.16900000 & 4.71500000 \\
\hline $\mathrm{H}$ & -3.80800000 & 4.44300000 & 4.77100000 \\
\hline $\mathrm{C}$ & -4.52100000 & 3.93100000 & 2.76700000 \\
\hline $\mathrm{H}$ & -4.55200000 & 2.99600000 & 2.20200000 \\
\hline $\mathrm{H}$ & -3.76500000 & 4.58200000 & 2.32100000 \\
\hline $\mathrm{C}$ & -5.89600000 & 4.58600000 & 2.59500000 \\
\hline $\mathrm{O}$ & -6.91400000 & 3.93700000 & 2.99200000 \\
\hline $\mathrm{O}$ & .94800000 & 5.71200000 & 2.0180 \\
\hline
\end{tabular}

Coordinate of ${ }^{3} \mathrm{IM} 3 \mathrm{a}^{\prime}$

$\begin{array}{cccc}\mathrm{C} & 0.13200000 & 1.51100000 & 10.78500000 \\ \mathrm{H} & 0.76700000 & 0.62300000 & 10.78800000 \\ \mathrm{H} & -0.66600000 & 1.34600000 & 11.51500000 \\ & & & \mathrm{~S} 47\end{array}$




\begin{tabular}{|c|c|c|c|}
\hline & -1.55200000 & 2.49000000 & 000 \\
\hline $\mathrm{H}$ & -2.18300000 & 2.88500000 & 9.84000000 \\
\hline & -0.40800000 & 1.75400000 & 9.40900000 \\
\hline & -1.66800000 & 2.61600000 & 7.79900000 \\
\hline $\mathrm{H}$ & -2.47900000 & 3.11200000 & 7.29100000 \\
\hline & -0.66600000 & 1.99800000 & 7.19300000 \\
\hline & 0.12600000 & 1.45700000 & 000000 \\
\hline $\mathrm{H}$ & 1.01900000 & 0.90500000 & 7.93500000 \\
\hline C & 3.74600000 & -0.21100000 & 4.84300000 \\
\hline & 4.50000000 & 0.49200000 & 4.46700000 \\
\hline $\mathrm{H}$ & 3.85300000 & -1.11000000 & 4.22700000 \\
\hline $\mathrm{C}$ & 2.35300000 & 0.36500000 & 4.50000000 \\
\hline ) & 1.62700000 & 0.90600000 & 5.39300000 \\
\hline $\mathrm{O}$ & 2.05900000 & 0.26000000 & 3.27400000 \\
\hline $\mathrm{C}$ & 0.59800000 & 7.17600000 & 6.70600000 \\
\hline $\mathrm{H}$ & -0.28800000 & 7.57500000 & 6.20500000 \\
\hline $\mathrm{H}$ & 1.44000000 & 7.81500000 & 6.41500000 \\
\hline $\mathrm{N}$ & 2.04700000 & 5.09200000 & 6.38200000 \\
\hline $\mathrm{H}$ & 2.92300000 & 5.42100000 & 6.80000000 \\
\hline $\mathrm{C}$ & 0.83500000 & 5.76400000 & 6.27000000 \\
\hline $\mathrm{C}$ & 1.88500000 & 3.85400000 & 0000 \\
\hline $\mathrm{H}$ & 2.64500000 & 00000 & 00000 \\
\hline $\mathrm{N}$ & 0.65700000 & 3.69200000 & 5.40900000 \\
\hline $\mathrm{C}$ & -0.00700000 & 4.87700000 & 5.65100000 \\
\hline $\mathrm{H}$ & -1.03600000 & 4.9970 & 5.35600000 \\
\hline $\mathrm{C}$ & -3.88300000 & -1.75600000 & 2.03400000 \\
\hline $\mathrm{O}$ & -4.21000000 & -0.81800000 & 1.32400000 \\
\hline $\mathrm{N}$ & -2.89200000 & -1.93400000 & 2.95000000 \\
\hline $\mathrm{C}$ & -1.77500000 & -1.18700000 & 3.50200000 \\
\hline $\mathrm{H}$ & -1.63800000 & -0.26500000 & 2.92500000 \\
\hline $\mathrm{C}$ & -2.10400000 & -0.77200000 & 4.95400000 \\
\hline $\mathrm{O}$ & -3.125 & -1.25 & 5.48400000 \\
\hline $\mathrm{O}$ & -1.25300000 & -0.04000000 & 5.55600000 \\
\hline $\mathrm{C}$ & -0.56500000 & -2.11200000 & 3.43300000 \\
\hline $\mathrm{C}$ & -0.63000000 & -3.16300000 & 2.40100000 \\
\hline $\mathrm{H}$ & 0.54600000 & 1.02800000 & 3.23900000 \\
\hline $\mathrm{H}$ & -0.94000000 & -2.79800000 & 1.42000000 \\
\hline $\mathrm{H}$ & 0.31100000 & -3.70400000 & 2.30400000 \\
\hline $\mathrm{C}$ & 0.29700000 & -2.37800000 & 4.62200000 \\
\hline $\mathrm{H}$ & -0.23300000 & -3.03300000 & 5.33300000 \\
\hline $\mathrm{H}$ & 1.20600000 & -2.90700000 & 4.32800000 \\
\hline $\mathrm{H}$ & 0.55700000 & -1.46900000 & 5.16000000 \\
\hline S & -1.89400000 & -4.49100000 & 2.91100000 \\
\hline $\mathrm{C}$ & -3.24600000 & -3.29900000 & 3.31100000 \\
\hline $\mathrm{H}$ & -3.53000000 & -3.40700000 & $\begin{array}{c}4.35600000 \\
\text { S48 }\end{array}$ \\
\hline
\end{tabular}




\begin{tabular}{|c|c|c|c|}
\hline $\mathrm{C}$ & -4.44100000 & -3.17000000 & 2.31100000 \\
\hline $\mathrm{H}$ & -5.39200000 & -3.14500000 & 2.84600000 \\
\hline $\mathrm{N}$ & -4.52400000 & -4.11600000 & 1.23500000 \\
\hline $\mathrm{H}$ & -3.90600000 & -4.01000000 & 0.44300000 \\
\hline $\mathrm{C}$ & -4.89400000 & -5.39300000 & 1.59000000 \\
\hline $\mathrm{O}$ & -5.34300000 & -5.59000000 & 2.72100000 \\
\hline $\mathrm{C}$ & -4.81700000 & -6.47500000 & 0.54900000 \\
\hline $\mathrm{H}$ & -5.78500000 & -6.47100000 & 0.03100000 \\
\hline $\mathrm{H}$ & -4.75100000 & -7.42100000 & 1.09500000 \\
\hline $\mathrm{C}$ & -3.73100000 & -6.36900000 & -0.52200000 \\
\hline $\mathrm{H}$ & -3.91200000 & -5.47800000 & -1.13600000 \\
\hline $\mathrm{H}$ & -2.74600000 & -6.25400000 & -0.05500000 \\
\hline $\mathrm{C}$ & -3.72500000 & -7.60200000 & -1.43800000 \\
\hline $\mathrm{H}$ & -3.09400000 & -7.40600000 & -2.31400000 \\
\hline $\mathrm{H}$ & -3.25900000 & -8.44500000 & -0.92100000 \\
\hline $\mathrm{N}$ & -5.86400000 & -6.89900000 & -2.45800000 \\
\hline $\mathrm{H}$ & -5.40000000 & -6.64700000 & -3.34300000 \\
\hline $\mathrm{H}$ & -6.72800000 & -7.32100000 & -2.79900000 \\
\hline $\mathrm{C}$ & -5.11900000 & -8.03500000 & -1.90700000 \\
\hline $\mathrm{H}$ & -5.68300000 & -8.40800000 & -1.03800000 \\
\hline $\mathrm{C}$ & -5.04400000 & -9.19800000 & -2.91700000 \\
\hline $\mathrm{O}$ & -4.05400000 & -9.97300000 & -2.81700000 \\
\hline $\mathrm{O}$ & -5.94300000 & -9.26100000 & -3.78800000 \\
\hline $\mathrm{Fe}$ & -0.42700000 & 1.74900000 & 5.17200000 \\
\hline $\mathrm{O}$ & -0.22700000 & 1.62600000 & 3.39300000 \\
\hline $\mathrm{C}$ & -3.09800000 & 2.52800000 & 4.21200000 \\
\hline $\mathrm{O}$ & -3.27000000 & 1.46700000 & 3.59400000 \\
\hline $\mathrm{O}$ & -2.08200000 & 2.81500000 & 4.96800000 \\
\hline $\mathrm{C}$ & -4.16900000 & 3.61900000 & 4.13800000 \\
\hline $\mathrm{H}$ & -5.04700000 & 3.22900000 & 4.67000000 \\
\hline $\mathrm{H}$ & -3.82600000 & 4.51300000 & 4.66500000 \\
\hline $\mathrm{C}$ & -4.57600000 & 3.94100000 & 2.69100000 \\
\hline $\mathrm{H}$ & -4.63300000 & 2.98800000 & 2.15900000 \\
\hline $\mathrm{H}$ & -3.82400000 & 4.56900000 & 2.20700000 \\
\hline $\mathrm{C}$ & -5.94600000 & 4.60900000 & 2.55600000 \\
\hline $\mathrm{O}$ & -6.96200000 & 3.95600000 & 2.95200000 \\
\hline $\mathrm{O}$ & -5.99800000 & 5.75400000 & 2.01700000 \\
\hline \multicolumn{4}{|c|}{ Coordinate of ${ }^{3} \mathrm{TS} 4 \mathrm{a}^{\prime}$} \\
\hline $\mathrm{C}$ & 0.13700000 & 1.51900000 & 10.88800000 \\
\hline $\mathrm{H}$ & 0.76800000 & 0.62700000 & 10.88700000 \\
\hline $\mathrm{H}$ & -0.64500000 & 1.36400000 & 11.63800000 \\
\hline $\mathrm{N}$ & -1.57300000 & 2.48900000 & 9.26200000 \\
\hline $\mathrm{H}$ & -2.20400000 & 2.87800000 & 9.96700000 \\
\hline $\mathrm{C}$ & -0.42500000 & 1.75700000 & 9.52200000 \\
\hline
\end{tabular}




\begin{tabular}{|c|c|c|c|}
\hline & -1.67600000 & 2.63800000 & 000 \\
\hline $\mathrm{H}$ & -2.48900000 & 3.14100000 & 7.42000000 \\
\hline & -0.66900000 & 2.04000000 & 7.30200000 \\
\hline & 0.11200000 & 1.48700000 & 8.28800000 \\
\hline & 1.01200000 & 0.94300000 & 8.04700000 \\
\hline & 3.83200000 & -0.17000000 & 4.78500000 \\
\hline & 4.58200000 & 0.56500000 & 00000 \\
\hline H & 3.99100000 & -1.04700000 & 4.15000000 \\
\hline & 2.43300000 & 0.37800000 & 4.41400000 \\
\hline & 1.70600000 & 0.94200000 & 5.28100000 \\
\hline & 2.13500000 & 0.21100000 & 3.19200000 \\
\hline $\mathrm{C}$ & 0.58200000 & 7.17100000 & 6.70900000 \\
\hline $\mathrm{H}$ & -0.30400000 & 7.58100000 & 6.21600000 \\
\hline I & 1.42600000 & 7.80800000 & 6.41 \\
\hline N & 2.02000000 & 5.08600000 & 6.35600000 \\
\hline $\mathrm{H}$ & 2.89400000 & 5.40600000 & 6.7840000 \\
\hline $\mathrm{C}$ & 0.81000000 & 5.76500000 & 6.25500000 \\
\hline $\mathrm{C}$ & 1.86400000 & 3.87200000 & 5.78800000 \\
\hline $\mathrm{H}$ & 2.62400000 & 3.10800000 & 5.76900000 \\
\hline $\mathrm{N}$ & 0.63900000 & 3.72600000 & 5.30800000 \\
\hline $\mathrm{C}$ & -0.02600000 & 4.90200000 & 5.59300000 \\
\hline $\mathrm{H}$ & -1.05600000 & 5.02900000 & 5.30300000 \\
\hline $\mathrm{C}$ & -3.70700000 & -1.60700000 & 1.96500000 \\
\hline $\mathrm{O}$ & -4.01200000 & -0.65100000 & 1.27700000 \\
\hline I & -2.73000000 & -1.84100000 & 2.89500000 \\
\hline$C$ & -1.64500000 & -1.20100000 & 3.60900000 \\
\hline $\mathrm{H}$ & -1.26032410 & -0.12145863 & 3.19065221 \\
\hline $\mathrm{C}$ & -2.10100000 & -0.80000000 & 5.05900000 \\
\hline $\mathrm{O}$ & -3.17600000 & -1.29200000 & .45900000 \\
\hline $\mathrm{O}$ & -1.29800000 & -0.09700000 & 5.72500000 \\
\hline $\mathrm{C}$ & -0.53000000 & -2.14300000 & 3.61000000 \\
\hline $\mathrm{C}$ & -0.53800000 & -3.21200000 & 2.58800000 \\
\hline $\mathrm{H}$ & 0.48100000 & 0.72400000 & 3.08700000 \\
\hline $\mathrm{H}$ & -0.75300000 & -2.82700000 & 1.58900000 \\
\hline $\mathrm{H}$ & 0.39300000 & -3.77800000 & 2.56000000 \\
\hline $\mathrm{C}$ & 0.48400000 & -2.21400000 & 4.69000000 \\
\hline $\mathrm{H}$ & 0.16500000 & -2.99100000 & 5.40300000 \\
\hline $\mathrm{H}$ & 1.45400000 & -2.53400000 & 4.31300000 \\
\hline $\mathrm{H}$ & 0.57200000 & -1.28400000 & 5.24300000 \\
\hline $\mathrm{S}$ & -1.88600000 & -4.44400000 & 3.02000000 \\
\hline $\mathrm{C}$ & -3.19900000 & -3.17900000 & 3.26400000 \\
\hline $\mathrm{H}$ & -3.55100000 & -3.22800000 & 4.29000000 \\
\hline $\mathrm{C}$ & -4.32500000 & -3.00600000 & 2.20800000 \\
\hline $\mathrm{H}$ & -5.29600000 & -2.93100000 & 2.70200000 \\
\hline $\mathrm{N}$ & -4.39800000 & -3.96700000 & $\begin{array}{c}1.14200000 \\
\text { S50 }\end{array}$ \\
\hline
\end{tabular}




\begin{tabular}{|c|c|c|c|}
\hline $\mathrm{H}$ & -3.84900000 & -3.84000000 & 0.30400000 \\
\hline $\mathrm{C}$ & -4.73900000 & -5.24300000 & 1.53200000 \\
\hline $\mathrm{O}$ & -5.11300000 & -5.41700000 & 2.69400000 \\
\hline $\mathrm{C}$ & -4.72100000 & -6.34800000 & 0.51300000 \\
\hline $\mathrm{H}$ & -5.69600000 & -6.33000000 & 0.01000000 \\
\hline $\mathrm{H}$ & -4.67100000 & -7.28100000 & 1.08200000 \\
\hline $\mathrm{C}$ & -3.65100000 & -6.30600000 & -0.58100000 \\
\hline $\mathrm{H}$ & -3.82300000 & -5.43400000 & -1.22400000 \\
\hline $\mathrm{H}$ & -2.65600000 & -6.19600000 & -0.13600000 \\
\hline $\mathrm{C}$ & -3.68300000 & -7.57400000 & -1.44900000 \\
\hline $\mathrm{H}$ & -3.06400000 & -7.42100000 & -2.34100000 \\
\hline $\mathrm{H}$ & -3.22000000 & -8.40400000 & -0.90800000 \\
\hline $\mathrm{N}$ & -5.84700000 & -6.86800000 & -2.41500000 \\
\hline $\mathrm{H}$ & -5.40900000 & -6.62600000 & -3.31500000 \\
\hline $\mathrm{H}$ & -6.72400000 & -7.28900000 & -2.72400000 \\
\hline $\mathrm{C}$ & -5.09000000 & -8.00500000 & -1.88000000 \\
\hline $\mathrm{H}$ & -5.63200000 & -8.37400000 & -0.99600000 \\
\hline $\mathrm{C}$ & -5.04100000 & -9.17100000 & -2.89300000 \\
\hline $\mathrm{O}$ & -4.05800000 & -9.95700000 & -2.80700000 \\
\hline $\mathrm{O}$ & -5.95000000 & -9.22100000 & -3.75300000 \\
\hline $\mathrm{Fe}$ & -0.47800000 & 1.75100000 & 5.11400000 \\
\hline $\mathrm{O}$ & -0.39100000 & 1.16700000 & 3.23200000 \\
\hline $\mathrm{C}$ & -3.16900000 & 2.54400000 & 4.23100000 \\
\hline $\mathrm{O}$ & -3.33900000 & 1.44300000 & 3.68300000 \\
\hline $\mathrm{O}$ & -2.12500000 & 2.89000000 & 4.91000000 \\
\hline $\mathrm{C}$ & -4.26100000 & 3.60700000 & 4.12200000 \\
\hline $\mathrm{H}$ & -5.13700000 & 3.22400000 & 4.66100000 \\
\hline $\mathrm{H}$ & -3.93100000 & 4.52400000 & 4.61800000 \\
\hline $\mathrm{C}$ & -4.66400000 & 3.87600000 & 2.66400000 \\
\hline $\mathrm{H}$ & -4.74400000 & 2.90300000 & 2.17100000 \\
\hline $\mathrm{H}$ & -3.89800000 & 4.46700000 & 2.15300000 \\
\hline $\mathrm{C}$ & -6.01500000 & 4.57500000 & 2.52200000 \\
\hline $\mathrm{O}$ & -7.04700000 & 3.93300000 & 2.89700000 \\
\hline $\mathrm{O}$ & -6.03900000 & 5.73500000 & 2.01500000 \\
\hline \multicolumn{4}{|c|}{ Coordinate of ${ }^{3} \mathrm{~Pa}^{\prime}$} \\
\hline $\mathrm{C}$ & 0.14700000 & 1.50900000 & 10.95500000 \\
\hline $\mathrm{H}$ & 0.77800000 & 0.61800000 & 10.96600000 \\
\hline $\mathrm{H}$ & -0.62000000 & 1.37400000 & 11.72300000 \\
\hline $\mathrm{N}$ & -1.60400000 & 2.41800000 & 9.33700000 \\
\hline $\mathrm{H}$ & -2.22300000 & 2.82600000 & 10.04300000 \\
\hline $\mathrm{C}$ & -0.44500000 & 1.70400000 & 9.59600000 \\
\hline $\mathrm{C}$ & -1.73900000 & 2.53000000 & 7.99400000 \\
\hline $\mathrm{H}$ & -2.56200000 & 3.01100000 & 7.49100000 \\
\hline $\mathrm{N}$ & -0.73800000 & 1.91900000 & $\begin{array}{c}7.38100000 \\
\text { S51 }\end{array}$ \\
\hline
\end{tabular}




\begin{tabular}{|c|c|c|c|}
\hline $\mathrm{C}$ & 0.07300000 & 00000 & 000 \\
\hline $\mathrm{H}$ & 0.97600000 & 0.86500000 & 8.11300000 \\
\hline $\mathrm{C}$ & 3.73900000 & -0.34100000 & 4.82800000 \\
\hline $\mathrm{H}$ & 4.50400000 & 0.32600000 & 4.41100000 \\
\hline $\mathrm{H}$ & 3.80700000 & -1.26200000 & 4.23800000 \\
\hline $\mathrm{C}$ & 2.35800000 & 0.28300000 & 4.52700000 \\
\hline $\mathrm{O}$ & 1.67000000 & 0.80000000 & 5.44100000 \\
\hline $\mathrm{O}$ & 2.03600000 & 0.24300000 & 3.28600000 \\
\hline $\mathrm{C}$ & 0.58600000 & 7.11000000 & 6.69800000 \\
\hline $\mathrm{H}$ & -0.29700000 & 7.50600000 & 6.18800000 \\
\hline $\mathrm{H}$ & 1.43500000 & 7.73000000 & 6.38700000 \\
\hline $\mathrm{N}$ & 2.01100000 & 5.00500000 & 6.40800000 \\
\hline $\mathrm{H}$ & 2.89900000 & 5.34600000 & 6.78800000 \\
\hline $\mathrm{C}$ & 0.80200000 & 5.68300000 & 6.30500000 \\
\hline $\mathrm{C}$ & 1.82400000 & 3.75000000 & 5.94700000 \\
\hline $\mathrm{H}$ & 2.57900000 & 2.98100000 & 5.95300000 \\
\hline $\mathrm{N}$ & 0.58000000 & 3.58000000 & 5.52700000 \\
\hline $\mathrm{C}$ & -0.06400000 & 4.78100000 & 5.74600000 \\
\hline $\mathrm{H}$ & -1.09800000 & 4.90300000 & 5.47000000 \\
\hline $\mathrm{C}$ & -3.86100000 & -2.00700000 & 2.19600000 \\
\hline $\mathrm{O}$ & -4.07900000 & -1.02000000 & 1.52300000 \\
\hline $\mathrm{N}$ & -2.93100000 & -2.29900000 & 3.16000000 \\
\hline $\mathrm{C}$ & -1.86800000 & -1.72700000 & 3.89500000 \\
\hline $\mathrm{H}$ & 0.20900000 & 0.74100000 & 3.22600000 \\
\hline $\mathrm{C}$ & -2.29900000 & -0.81600000 & 5.02300000 \\
\hline $\mathrm{O}$ & -3.49200000 & -0.97700000 & 5.38400000 \\
\hline $\mathrm{O}$ & -1.43600000 & -0.07900000 & 5.57900000 \\
\hline $\mathrm{C}$ & -0.72700000 & -2.45100000 & 3.82500000 \\
\hline $\mathrm{C}$ & -0.68300000 & -3.57500000 & 2.82500000 \\
\hline $\mathrm{H}$ & -1.35939961 & 1.31645452 & 3.21708339 \\
\hline $\mathrm{H}$ & -0.79100000 & -3.19300000 & 1.80800000 \\
\hline $\mathrm{H}$ & 0.25500000 & -4.13000000 & 2.87400000 \\
\hline $\mathrm{C}$ & 0.41500000 & -2.33000000 & 4.78000000 \\
\hline $\mathrm{H}$ & 0.37800000 & -3.17100000 & 5.48500000 \\
\hline $\mathrm{H}$ & 1.37500000 & -2.39800000 & 4.27300000 \\
\hline $\mathrm{H}$ & 0.36700000 & -1.40200000 & 5.34000000 \\
\hline $\mathrm{S}$ & -2.03400000 & -4.84600000 & 3.09300000 \\
\hline $\mathrm{C}$ & -3.38800000 & -3.65600000 & 3.42600000 \\
\hline $\mathrm{H}$ & -3.75600000 & -3.79200000 & 4.44400000 \\
\hline $\mathrm{C}$ & -4.50400000 & -3.40700000 & 2.36500000 \\
\hline $\mathrm{H}$ & -5.48000000 & -3.35700000 & 2.85100000 \\
\hline $\mathrm{N}$ & -4.57300000 & -4.29100000 & 1.24300000 \\
\hline $\mathrm{H}$ & -3.92600000 & -4.16700000 & 0.47700000 \\
\hline $\mathrm{C}$ & -5.01500000 & -5.56300000 & 1.51900000 \\
\hline $\mathrm{O}$ & -5.52800000 & -5.79700000 & $\begin{array}{c}2.61500000 \\
\text { S52 }\end{array}$ \\
\hline
\end{tabular}




\begin{tabular}{lrrc}
$\mathrm{C}$ & -4.91300000 & -6.59300000 & 0.43600000 \\
$\mathrm{H}$ & -5.87900000 & -6.58800000 & -0.08400000 \\
$\mathrm{H}$ & -4.82900000 & -7.56200000 & 0.93700000 \\
$\mathrm{C}$ & -3.82100000 & -6.40700000 & -0.61700000 \\
$\mathrm{H}$ & -4.02500000 & -5.49700000 & -1.19400000 \\
$\mathrm{H}$ & -2.84200000 & -6.28600000 & -0.13800000 \\
$\mathrm{C}$ & -3.78300000 & -7.60100000 & -1.57400000 \\
$\mathrm{H}$ & -3.17100000 & -7.35800000 & -2.45200000 \\
$\mathrm{H}$ & -3.28900000 & -8.44700000 & -1.09300000 \\
$\mathrm{~N}$ & -5.93400000 & -6.95700000 & -2.61600000 \\
$\mathrm{H}$ & -5.45600000 & -6.69200000 & -3.4890000 \\
$\mathrm{H}$ & -6.77800000 & -7.40400000 & -2.97700000 \\
$\mathrm{C}$ & -5.16900000 & -8.06400000 & -2.03500000 \\
$\mathrm{H}$ & -5.72800000 & -8.43100000 & -1.16200000 \\
$\mathrm{C}$ & -5.05500000 & -9.25200000 & -3.00200000 \\
$\mathrm{O}$ & -4.05900000 & -10.00900000 & -2.83700000 \\
$\mathrm{O}$ & -5.92500000 & -9.35500000 & -3.89700000 \\
$\mathrm{Fe}$ & -0.51200000 & 1.72900000 & 5.41500000 \\
$\mathrm{O}$ & -0.40800000 & 1.48200000 & 3.40600000 \\
$\mathrm{C}$ & -3.04600000 & 2.72100000 & 4.05300000 \\
$\mathrm{O}$ & -2.91700000 & 1.81900000 & 3.15900000 \\
$\mathrm{O}$ & -2.27800000 & 2.87900000 & 5.04500000 \\
$\mathrm{C}$ & -4.23300000 & 3.67400000 & 3.96200000 \\
$\mathrm{H}$ & -5.05200000 & 3.21800000 & 4.53400000 \\
$\mathrm{H}$ & -3.95900000 & 4.59700000 & 4.48100000 \\
$\mathrm{C}$ & -4.70300000 & 3.95100000 & 2.53500000 \\
$\mathrm{H}$ & -4.81500000 & 2.98300000 & 2.03600000 \\
$\mathrm{H}$ & -3.95300000 & 4.53200000 & 1.99100000 \\
$\mathrm{C}$ & -6.04800000 & 4.66800000 & 2.45400000 \\
$\mathrm{O}$ & -7.07700000 & 4.04600000 & 2.86400000 \\
$\mathrm{O}$ & -6.06000000 & 5.83100000 & 1.95500000 \\
& & & \\
\hline
\end{tabular}

Coordinate of ${ }^{5} \mathrm{TS} 1 \mathrm{a}$

$\begin{array}{lrrr}\mathrm{C} & 0.135 & 1.454 & 10.736 \\ \mathrm{H} & 0.787 & 0.578 & 10.748 \\ \mathrm{H} & -0.662 & 1.277 & 11.464 \\ \mathrm{~N} & -1.590 & 2.348 & 9.088 \\ \mathrm{H} & -2.205 & 2.764 & 9.789 \\ \mathrm{C} & -0.411 & 1.669 & 9.358 \\ \mathrm{C} & -1.728 & 2.422 & 7.740 \\ \mathrm{H} & -2.561 & 2.886 & 7.238 \\ \mathrm{~N} & -0.718 & 1.822 & 7.130 \\ \mathrm{C} & 0.109 & 1.355 & 8.126 \\ \mathrm{H} & 1.023 & 0.834 & 7.889 \\ \mathrm{C} & 3.890 & 0.140 & 4.670\end{array}$




\begin{tabular}{|c|c|c|c|}
\hline $\mathrm{H}$ & 4.745 & 0.752 & .362 \\
\hline $\mathrm{H}$ & 3.908 & -0.741 & 4.019 \\
\hline $\mathrm{C}$ & 2.606 & 0.922 & 4.284 \\
\hline U & 1.665 & 1.040 & 5.152 \\
\hline $\mathrm{O}$ & 2.604 & 1.389 & 3.128 \\
\hline C & 0.494 & 7.112 & 6.674 \\
\hline $\mathrm{H}$ & -0.402 & 7.518 & 6.198 \\
\hline $\mathrm{H}$ & 1.332 & 7.739 & 6.347 \\
\hline N & 1.936 & 5.047 & 6.289 \\
\hline $\mathrm{H}$ & 2.814 & 5.385 & 6.694 \\
\hline C & 0.708 & 5.696 & 6.239 \\
\hline C & 1.782 & 3.822 & 5.749 \\
\hline $\mathrm{H}$ & 2.562 & 3.084 & 5.710 \\
\hline $\mathrm{N}$ & 0.538 & 3.638 & 5.335 \\
\hline C & -0.140 & 4.800 & 5.640 \\
\hline $\mathrm{H}$ & -1.181 & 4.913 & 5.384 \\
\hline $\mathrm{C}$ & -4.108 & -2.857 & 3.935 \\
\hline $\mathrm{O}$ & -5.038 & -2.279 & 4.482 \\
\hline $\mathrm{N}$ & -2.801 & -2.524 & 3.733 \\
\hline $\mathrm{C}$ & -2.002 & -1.329 & 3.575 \\
\hline $\mathrm{H}$ & -2.624 & -0.435 & 3.652 \\
\hline $\mathrm{C}$ & -0.945 & -1.359 & 4.700 \\
\hline $\mathrm{O}$ & -0.406 & -2.465 & 4.918 \\
\hline $\mathrm{O}$ & -0.762 & -0.297 & 5.372 \\
\hline $\mathrm{C}$ & -1.370 & -1.416 & 2.114 \\
\hline $\mathrm{C}$ & -1.568 & -0.106 & 1.421 \\
\hline $\mathrm{H}$ & -0.979 & 0.694 & 2.253 \\
\hline $\mathrm{H}$ & -2.589 & 0.259 & 1.381 \\
\hline $\mathrm{H}$ & -1.029 & -0.000 & 0.481 \\
\hline $\mathrm{C}$ & 0.116 & -1.793 & 2.045 \\
\hline $\mathrm{H}$ & 0.312 & -2.775 & 2.478 \\
\hline $\mathrm{H}$ & 0.430 & -1.817 & 1.000 \\
\hline $\mathrm{H}$ & 0.702 & -1.011 & 2.543 \\
\hline $\mathrm{S}$ & -2.354 & -2.796 & 1.225 \\
\hline $\mathrm{C}$ & -2.493 & -3.637 & 2.862 \\
\hline $\mathrm{H}$ & -1.585 & -4.187 & 3.108 \\
\hline $\mathrm{C}$ & -3.851 & -4.250 & 3.298 \\
\hline $\mathrm{H}$ & -3.660 & -4.973 & 4.093 \\
\hline $\mathrm{N}$ & -4.674 & -4.893 & 2.323 \\
\hline $\mathrm{H}$ & -5.180 & -4.339 & 1.647 \\
\hline $\mathrm{C}$ & -4.268 & -6.167 & 1.979 \\
\hline $\mathrm{O}$ & -3.481 & -6.771 & 2.714 \\
\hline $\mathrm{C}$ & -4.728 & -6.745 & 0.681 \\
\hline $\mathrm{H}$ & -5.696 & -6.355 & 0.359 \\
\hline $\mathrm{H}$ & -4.831 & -7.819 & 0.854 \\
\hline
\end{tabular}




$\begin{array}{lccc}\mathrm{C} & -3.681 & -6.485 & -0.429 \\ \mathrm{H} & -3.868 & -5.498 & -0.866 \\ \mathrm{H} & -2.677 & -6.461 & 0.009 \\ \mathrm{C} & -3.721 & -7.574 & -1.506 \\ \mathrm{H} & -3.158 & -7.238 & -2.388 \\ \mathrm{H} & -3.202 & -8.462 & -1.141 \\ \mathrm{~N} & -5.884 & -6.865 & -2.466 \\ \mathrm{H} & -5.437 & -6.613 & -3.359 \\ \mathrm{H} & -6.754 & -7.288 & -2.791 \\ \mathrm{C} & -5.122 & -8.001 & -1.939 \\ \mathrm{H} & -5.665 & -8.382 & -1.062 \\ \mathrm{C} & -5.054 & -9.174 & -2.942 \\ \mathrm{O} & -4.078 & -9.967 & -2.819 \\ \mathrm{O} & -5.944 & -9.230 & -3.821 \\ \mathrm{Fe} & -0.334 & 1.619 & 4.890 \\ \mathrm{O} & -0.371 & 1.360 & 3.135 \\ \mathrm{C} & -3.147 & 2.550 & 4.077 \\ \mathrm{O} & -3.359 & 1.648 & 3.231 \\ \mathrm{O} & -2.132 & 2.632 & 4.857 \\ \mathrm{C} & -4.194 & 3.648 & 4.230 \\ \mathrm{H} & -5.149 & 3.150 & 4.426 \\ \mathrm{H} & -3.944 & 4.264 & 5.098 \\ \mathrm{C} & -4.374 & 4.519 & 2.976 \\ \mathrm{H} & -3.947 & 4.013 & 2.107 \\ \mathrm{H} & -3.827 & 5.459 & 3.053 \\ \mathrm{C} & -5.824 & 4.833 & 2.586 \\ \mathrm{O} & -6.753 & 4.025 & 2.884 \\ \mathrm{O} & -5.992 & 5.904 & 1.932\end{array}$

Coordinate of ${ }^{5} \mathrm{IM} 1 \mathrm{a}$

$\begin{array}{lrrr}\mathrm{C} & 0.175 & 1.454 & 10.865 \\ \mathrm{H} & 0.837 & 0.587 & 10.885 \\ \mathrm{H} & -0.601 & 1.289 & 11.618 \\ \mathrm{~N} & -1.611 & 2.248 & 9.228 \\ \mathrm{H} & -2.228 & 2.669 & 9.928 \\ \mathrm{C} & -0.407 & 1.616 & 9.496 \\ \mathrm{C} & -1.773 & 2.286 & 7.883 \\ \mathrm{H} & -2.622 & 2.719 & 7.380 \\ \mathrm{~N} & -0.752 & 1.703 & 7.272 \\ \mathrm{C} & 0.106 & 1.289 & 8.267 \\ \mathrm{H} & 1.039 & 0.800 & 8.033 \\ \mathrm{C} & 3.860 & -0.045 & 4.774 \\ \mathrm{H} & 4.692 & 0.575 & 4.421 \\ \mathrm{H} & 3.889 & -0.953 & 4.163 \\ \mathrm{C} & 2.557 & 0.681 & 4.357\end{array}$




\begin{tabular}{|c|c|c|c|}
\hline $\mathrm{O}$ & 1.653 & 0.936 & 5.241 \\
\hline O & 2.506 & 0.976 & 3.150 \\
\hline & 0.511 & 7.082 & 6.691 \\
\hline $\mathrm{H}$ & -0.380 & 7.483 & 6.201 \\
\hline $\mathrm{H}$ & 1.355 & 7.699 & 6.359 \\
\hline $\mathrm{N}$ & 1.947 & 5.003 & 6.355 \\
\hline $\mathrm{H}$ & 2.834 & 5.359 & 6.724 \\
\hline C & 0.720 & 5.654 & 6.293 \\
\hline $\mathrm{C}$ & 1.780 & 3.752 & 5.885 \\
\hline $\mathrm{H}$ & 2.557 & 3.009 & 5.852 \\
\hline $\mathrm{N}$ & 0.528 & 3.554 & 5.507 \\
\hline $\mathrm{C}$ & -0.142 & 4.734 & 5.755 \\
\hline $\mathrm{H}$ & -1.186 & 4.836 & 5.508 \\
\hline $\mathrm{C}$ & -4.364 & -2.834 & 4.083 \\
\hline $\mathrm{O}$ & -5.309 & -2.181 & 4.515 \\
\hline $\mathrm{N}$ & -3.057 & -2.522 & 3.874 \\
\hline $\mathrm{C}$ & -2.261 & -1.360 & 3.541 \\
\hline $\mathrm{H}$ & -2.845 & -0.450 & 3.667 \\
\hline $\mathrm{C}$ & -1.083 & -1.358 & 4.549 \\
\hline $\mathrm{O}$ & -0.424 & -2.411 & 4.652 \\
\hline $\mathrm{O}$ & -0.964 & -0.327 & 5.289 \\
\hline $\mathrm{C}$ & -1.859 & -1.521 & 2.007 \\
\hline $\mathrm{C}$ & -2.664 & -0.622 & 1.175 \\
\hline $\mathrm{H}$ & -1.518 & 1.474 & 3.108 \\
\hline $\mathrm{H}$ & -3.694 & -0.395 & 1.426 \\
\hline $\mathrm{H}$ & -2.297 & -0.312 & 0.205 \\
\hline $\mathrm{C}$ & -0.362 & -1.405 & 1.704 \\
\hline $\mathrm{H}$ & 0.169 & -2.268 & 2.102 \\
\hline $\mathrm{H}$ & -0.209 & -1.396 & 0.621 \\
\hline $\mathrm{H}$ & 0.031 & -0.470 & 2.119 \\
\hline $\mathrm{S}$ & -2.418 & -3.331 & 1.507 \\
\hline $\mathrm{C}$ & -2.695 & -3.786 & 3.256 \\
\hline $\mathrm{H}$ & -1.825 & -4.272 & 3.691 \\
\hline $\mathrm{C}$ & -4.103 & -4.307 & 3.669 \\
\hline $\mathrm{H}$ & -4.023 & -4.938 & 4.556 \\
\hline $\mathrm{N}$ & -4.870 & -4.983 & 2.658 \\
\hline $\mathrm{H}$ & -5.479 & -4.453 & 2.051 \\
\hline $\mathrm{C}$ & -4.298 & -6.150 & 2.202 \\
\hline $\mathrm{O}$ & -3.389 & -6.659 & 2.868 \\
\hline $\mathrm{C}$ & -4.752 & -6.748 & 0.903 \\
\hline $\mathrm{H}$ & -5.713 & -6.352 & 0.568 \\
\hline $\mathrm{H}$ & -4.877 & -7.818 & 1.096 \\
\hline $\mathrm{C}$ & -3.693 & -6.539 & -0.208 \\
\hline $\mathrm{H}$ & -3.864 & -5.562 & -0.673 \\
\hline $\mathrm{H}$ & -2.695 & -6.516 & 0.243 \\
\hline
\end{tabular}




$\begin{array}{llll}\mathrm{C} & -3.726 & -7.648 & -1.266 \\ \mathrm{H} & -3.089 & -7.358 & -2.112 \\ \mathrm{H} & -3.273 & -8.551 & -0.852 \\ \mathrm{~N} & -5.810 & -6.824 & -2.305 \\ \mathrm{H} & -5.332 & -6.573 & -3.181 \\ \mathrm{H} & -6.688 & -7.203 & -2.662 \\ \mathrm{C} & -5.116 & -8.011 & -1.789 \\ \mathrm{H} & -5.718 & -8.402 & -0.954 \\ \mathrm{C} & -5.053 & -9.135 & -2.854 \\ \mathrm{O} & -4.073 & -9.931 & -2.798 \\ \mathrm{O} & -5.960 & -9.149 & -3.719 \\ \mathrm{Fe} & -0.328 & 1.567 & 5.079 \\ \mathrm{O} & -0.535 & 1.521 & 3.229 \\ \mathrm{C} & -3.136 & 2.614 & 4.179 \\ \mathrm{O} & -3.204 & 1.773 & 3.228 \\ \mathrm{O} & -2.227 & 2.626 & 5.068 \\ \mathrm{C} & -4.229 & 3.664 & 4.270 \\ \mathrm{H} & -5.169 & 3.133 & 4.455 \\ \mathrm{H} & -4.025 & 4.305 & 5.131 \\ \mathrm{C} & -4.407 & 4.490 & 2.987 \\ \mathrm{H} & -4.022 & 3.921 & 2.139 \\ \mathrm{H} & -3.819 & 5.408 & 3.012 \\ \mathrm{C} & -5.853 & 4.835 & 2.614 \\ \mathrm{O} & -6.790 & 4.035 & 2.912 \\ \mathrm{O} & -6.012 & 5.911 & 1.968\end{array}$

Coordinate of ${ }^{5} \mathrm{TS} 2 \mathrm{a}$

$\begin{array}{lrrr}\mathrm{C} & 0.191 & 1.457 & 10.870 \\ \mathrm{H} & 0.857 & 0.592 & 10.888 \\ \mathrm{H} & -0.581 & 1.290 & 11.628 \\ \mathrm{~N} & -1.609 & 2.242 & 9.245 \\ \mathrm{H} & -2.222 & 2.662 & 9.949 \\ \mathrm{C} & -0.401 & 1.615 & 9.506 \\ \mathrm{C} & -1.781 & 2.278 & 7.902 \\ \mathrm{H} & -2.635 & 2.707 & 7.404 \\ \mathrm{~N} & -0.762 & 1.697 & 7.285 \\ \mathrm{C} & 0.104 & 1.287 & 8.274 \\ \mathrm{H} & 1.036 & 0.800 & 8.033 \\ \mathrm{C} & 3.833 & -0.066 & 4.779 \\ \mathrm{H} & 4.661 & 0.552 & 4.412 \\ \mathrm{H} & 3.858 & -0.980 & 4.177 \\ \mathrm{C} & 2.524 & 0.651 & 4.367 \\ \mathrm{O} & 1.630 & 0.921 & 5.256 \\ \mathrm{O} & 2.457 & 0.925 & 3.155 \\ \mathrm{C} & 0.509 & 7.074 & 6.686\end{array}$




\begin{tabular}{|c|c|c|c|}
\hline $\mathrm{H}$ & -0.381 & 7.475 & 6.196 \\
\hline $\mathrm{H}$ & 1.354 & 7.689 & 6.351 \\
\hline N & 1.941 & 4.993 & 6.351 \\
\hline & 2.830 & 5.348 & 6.716 \\
\hline 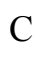 & 0.715 & 5.645 & 6.292 \\
\hline $\mathrm{C}$ & 1.770 & 3.740 & 5.887 \\
\hline H & 2.545 & 2.994 & 5.853 \\
\hline N & 0.515 & 3.542 & 5.517 \\
\hline C & -0.151 & 4.724 & 5.763 \\
\hline $\mathrm{H}$ & -1.196 & 4.826 & 5.520 \\
\hline C & -4.342 & -2.835 & 4.058 \\
\hline $\mathrm{O}$ & -5.268 & -2.186 & 4.538 \\
\hline $\mathrm{N}$ & -3.056 & -2.513 & 3.772 \\
\hline $\mathrm{C}$ & -2.260 & -1.330 & 3.521 \\
\hline $\mathrm{H}$ & -2.866 & -0.439 & 3.684 \\
\hline $\mathrm{C}$ & -1.107 & -1.353 & 4.558 \\
\hline $\mathrm{O}$ & -0.456 & -2.411 & 4.657 \\
\hline $\mathrm{O}$ & -0.996 & -0.332 & 5.314 \\
\hline $\mathrm{C}$ & -1.812 & -1.360 & 2.019 \\
\hline $\mathrm{C}$ & -2.631 & -0.587 & 1.173 \\
\hline $\mathrm{H}$ & -1.540 & 1.475 & 3.126 \\
\hline $\mathrm{H}$ & -3.633 & -0.290 & 1.460 \\
\hline $\mathrm{H}$ & -2.319 & -0.386 & 0.156 \\
\hline $\mathrm{C}$ & -0.327 & -1.391 & 1.701 \\
\hline $\mathrm{H}$ & 0.127 & -2.311 & 2.067 \\
\hline $\mathrm{H}$ & -0.179 & -1.354 & 0.619 \\
\hline $\mathrm{H}$ & 0.154 & -0.511 & 2.148 \\
\hline $\mathrm{S}$ & -2.465 & -3.417 & 1.392 \\
\hline $\mathrm{C}$ & -2.697 & -3.781 & 3.159 \\
\hline $\mathrm{H}$ & -1.813 & -4.248 & 3.591 \\
\hline $\mathrm{C}$ & -4.086 & -4.307 & 3.642 \\
\hline $\mathrm{H}$ & -3.963 & -4.932 & 4.529 \\
\hline $\mathrm{N}$ & -4.887 & -5.002 & 2.671 \\
\hline $\mathrm{H}$ & -5.468 & -4.468 & 2.041 \\
\hline $\mathrm{C}$ & -4.318 & -6.174 & 2.217 \\
\hline $\mathrm{O}$ & -3.412 & -6.688 & 2.883 \\
\hline $\mathrm{C}$ & -4.772 & -6.771 & 0.918 \\
\hline $\mathrm{H}$ & -5.733 & -6.374 & 0.584 \\
\hline $\mathrm{H}$ & -4.897 & -7.841 & 1.108 \\
\hline $\mathrm{C}$ & -3.712 & -6.558 & -0.192 \\
\hline $\mathrm{H}$ & -3.887 & -5.583 & -0.658 \\
\hline $\mathrm{H}$ & -2.714 & -6.530 & 0.259 \\
\hline $\mathrm{C}$ & -3.740 & -7.667 & -1.250 \\
\hline $\mathrm{H}$ & -3.092 & -7.381 & -2.088 \\
\hline $\mathrm{H}$ & -3.296 & -8.573 & -0.831 \\
\hline
\end{tabular}




$\begin{array}{lccc}\mathrm{N} & -5.810 & -6.832 & -2.310 \\ \mathrm{H} & -5.322 & -6.581 & -3.181 \\ \mathrm{H} & -6.686 & -7.207 & -2.678 \\ \mathrm{C} & -5.126 & -8.022 & -1.788 \\ \mathrm{H} & -5.739 & -8.412 & -0.961 \\ \mathrm{C} & -5.056 & -9.144 & -2.856 \\ \mathrm{O} & -4.072 & -9.934 & -2.800 \\ \mathrm{O} & -5.959 & -9.158 & -3.724 \\ \mathrm{Fe} & -0.349 & 1.559 & 5.098 \\ \mathrm{O} & -0.558 & 1.509 & 3.249 \\ \mathrm{C} & -3.154 & 2.618 & 4.187 \\ \mathrm{O} & -3.222 & 1.779 & 3.233 \\ \mathrm{O} & -2.251 & 2.620 & 5.082 \\ \mathrm{C} & -4.242 & 3.672 & 4.274 \\ \mathrm{H} & -5.184 & 3.146 & 4.462 \\ \mathrm{H} & -4.034 & 4.315 & 5.132 \\ \mathrm{C} & -4.418 & 4.495 & 2.989 \\ \mathrm{H} & -4.032 & 3.924 & 2.142 \\ \mathrm{H} & -3.829 & 5.413 & 3.011 \\ \mathrm{C} & -5.864 & 4.838 & 2.614 \\ \mathrm{O} & -6.800 & 4.038 & 2.910 \\ \mathrm{O} & -6.023 & 5.916 & 1.969\end{array}$

Coordinate of ${ }^{5} \mathrm{IM} 2 \mathrm{a}$

$\begin{array}{llll}\text { C } & 0.190 & 1.468 & 10.912\end{array}$

$\begin{array}{llll}\mathrm{H} & 0.852 & 0.600 & 10.933\end{array}$

$\begin{array}{llll}\mathrm{H} & -0.583 & 1.307 & 11.669\end{array}$

$\begin{array}{llll}\mathrm{N} & -1.598 & 2.262 & 9.277\end{array}$

$\begin{array}{llll}\mathrm{H} & -2.217 & 2.679 & 9.978\end{array}$

$\begin{array}{llll}\text { C } & -0.396 & 1.627 & 9.546\end{array}$

$\begin{array}{llll}\text { C } & -1.754 & 2.313 & 7.933\end{array}$

$\begin{array}{llll}\mathrm{H} & -2.601 & 2.750 & 7.430\end{array}$

$\begin{array}{llll}\mathrm{N} & -0.730 & 1.733 & 7.322\end{array}$

$\begin{array}{llll}\text { C } & 0.121 & 1.307 & 8.316\end{array}$

$\begin{array}{llll}\mathrm{H} & 1.053 & 0.815 & 8.083\end{array}$

$\begin{array}{llll}\text { C } & 3.861 & -0.071 & 4.805\end{array}$

$\begin{array}{llll}\mathrm{H} & 4.702 & 0.523 & 4.430\end{array}$

$\begin{array}{llll}\mathrm{H} & 3.859 & -0.988 & 4.206\end{array}$

$\begin{array}{llll}\text { C } & 2.572 & 0.682 & 4.399\end{array}$

$\begin{array}{llll}\mathrm{O} & 1.677 & 0.948 & 5.291\end{array}$

$\begin{array}{llll}\text { O } & 2.521 & 0.985 & 3.194\end{array}$

$\begin{array}{llll}\text { C } & 0.530 & 7.109 & 6.707\end{array}$

$\begin{array}{llll}\mathrm{H} & -0.360 & 7.506 & 6.213\end{array}$

$\begin{array}{llll}\mathrm{H} & 1.373 & 7.730 & 6.382\end{array}$

$\begin{array}{llll}\mathrm{N} & 1.977 & 5.036 & 6.371\end{array}$ 


\begin{tabular}{|c|c|c|c|}
\hline & 2.866 & 5.394 & 735 \\
\hline $\mathrm{C}$ & 0.748 & 5.683 & 6.311 \\
\hline C & 1.812 & 3.780 & 5.914 \\
\hline H & 2.592 & 3.040 & 5.882 \\
\hline N & 0.558 & 3.576 & 5.546 \\
\hline C & -0.115 & 4.755 & 5.787 \\
\hline $\mathrm{H}$ & -1.160 & 4.850 & 5.545 \\
\hline $\mathrm{C}$ & -4.293 & -2.761 & 4.065 \\
\hline $\mathrm{O}$ & -5.206 & -2.127 & 4.591 \\
\hline $\mathrm{N}$ & -3.036 & -2.411 & 3.700 \\
\hline $\mathrm{C}$ & -2.244 & -1.205 & 3.540 \\
\hline $\mathrm{H}$ & -2.871 & -0.337 & 3.747 \\
\hline $\mathrm{C}$ & -1.107 & -1.273 & 4.594 \\
\hline $\mathrm{O}$ & -0.466 & -2.340 & 4.672 \\
\hline $\mathrm{O}$ & -0.988 & -0.274 & 5.375 \\
\hline $\mathrm{C}$ & -1.770 & -1.130 & 2.067 \\
\hline $\mathrm{C}$ & -2.642 & -0.616 & 1.156 \\
\hline $\mathrm{H}$ & -1.465 & 1.481 & 3.200 \\
\hline $\mathrm{H}$ & -3.650 & -0.326 & 1.428 \\
\hline $\mathrm{H}$ & -2.336 & -0.458 & 0.129 \\
\hline $\mathrm{C}$ & -0.323 & -1.373 & 1.724 \\
\hline $\mathrm{H}$ & 0.088 & -2.316 & 2.084 \\
\hline $\mathrm{H}$ & -0.166 & -1.328 & 0.643 \\
\hline $\mathrm{H}$ & 0.200 & -0.528 & 2.188 \\
\hline $\mathrm{S}$ & -2.493 & -3.437 & 1.327 \\
\hline $\mathrm{C}$ & -2.663 & -3.687 & 3.110 \\
\hline $\mathrm{H}$ & -1.762 & -4.119 & 3.541 \\
\hline $\mathrm{C}$ & -4.029 & -4.231 & 3.652 \\
\hline $\mathrm{H}$ & -3.861 & -4.845 & 4.539 \\
\hline $\mathrm{N}$ & -4.855 & -4.949 & 2.717 \\
\hline $\mathrm{H}$ & -5.444 & -4.420 & 2.089 \\
\hline $\mathrm{C}$ & -4.283 & -6.117 & 2.253 \\
\hline $\mathrm{O}$ & -3.361 & -6.622 & 2.903 \\
\hline $\mathrm{C}$ & -4.761 & -6.730 & 0.967 \\
\hline $\mathrm{H}$ & -5.718 & -6.323 & 0.635 \\
\hline $\mathrm{H}$ & -4.904 & -7.794 & 1.179 \\
\hline $\mathrm{C}$ & -3.706 & -6.560 & -0.156 \\
\hline $\mathrm{H}$ & -3.872 & -5.595 & -0.645 \\
\hline $\mathrm{H}$ & -2.706 & -6.532 & 0.290 \\
\hline $\mathrm{C}$ & -3.745 & -7.692 & -1.190 \\
\hline $\mathrm{H}$ & -3.073 & -7.438 & -2.021 \\
\hline $\mathrm{H}$ & -3.328 & -8.597 & -0.743 \\
\hline $\mathrm{N}$ & -5.791 & -6.821 & -2.270 \\
\hline $\mathrm{H}$ & -5.295 & -6.572 & -3.136 \\
\hline H & -6.668 & -7.184 & -2.646 \\
\hline
\end{tabular}




$\begin{array}{llll}\mathrm{C} & -5.128 & -8.025 & -1.750 \\ \mathrm{H} & -5.756 & -8.414 & -0.934 \\ \mathrm{C} & -5.057 & -9.134 & -2.833 \\ \mathrm{O} & -4.072 & -9.923 & -2.794 \\ \mathrm{O} & -5.964 & -9.139 & -3.698 \\ \mathrm{Fe} & -0.294 & 1.598 & 5.151 \\ \mathrm{O} & -0.483 & 1.519 & 3.301 \\ \mathrm{C} & -3.103 & 2.658 & 4.212 \\ \mathrm{O} & -3.165 & 1.812 & 3.264 \\ \mathrm{O} & -2.201 & 2.673 & 5.106 \\ \mathrm{C} & -4.201 & 3.704 & 4.291 \\ \mathrm{H} & -5.138 & 3.170 & 4.487 \\ \mathrm{H} & -3.998 & 4.357 & 5.143 \\ \mathrm{C} & -4.389 & 4.513 & 2.999 \\ \mathrm{H} & -3.998 & 3.939 & 2.158 \\ \mathrm{H} & -3.809 & 5.436 & 3.013 \\ \mathrm{C} & -5.838 & 4.841 & 2.622 \\ \mathrm{O} & -6.768 & 4.036 & 2.927 \\ \mathrm{O} & -6.006 & 5.911 & 1.968\end{array}$

\begin{tabular}{lccc}
\multicolumn{4}{l}{ Coordinate of } \\
5 TS3a \\
$\mathrm{C}$ & 0.229 & 1.431 & 10.766 \\
$\mathrm{H}$ & 0.905 & 0.574 & 10.790 \\
$\mathrm{H}$ & -0.569 & 1.237 & 11.489 \\
$\mathrm{~N}$ & -1.517 & 2.248 & 9.111 \\
$\mathrm{H}$ & -2.148 & 2.644 & 9.810 \\
$\mathrm{C}$ & -0.313 & 1.616 & 9.384 \\
$\mathrm{C}$ & -1.664 & 2.319 & 7.771 \\
$\mathrm{H}$ & -2.508 & 2.756 & 7.264 \\
$\mathrm{~N}$ & -0.623 & 1.758 & 7.164 \\
$\mathrm{C}$ & 0.226 & 1.322 & 8.156 \\
$\mathrm{H}$ & 1.158 & 0.838 & 7.910 \\
$\mathrm{C}$ & 3.860 & -0.043 & 4.848 \\
$\mathrm{H}$ & 4.600 & 0.712 & 4.550 \\
$\mathrm{H}$ & 4.039 & -0.905 & 4.200 \\
$\mathrm{C}$ & 2.452 & 0.476 & 4.430 \\
$\mathrm{O}$ & 1.681 & 1.012 & 5.306 \\
$\mathrm{O}$ & 2.225 & 0.339 & 3.212 \\
$\mathrm{C}$ & 0.542 & 7.161 & 6.692 \\
$\mathrm{H}$ & -0.339 & 7.586 & 6.204 \\
$\mathrm{H}$ & 1.397 & 7.779 & 6.391 \\
$\mathrm{~N}$ & 1.951 & 5.066 & 6.340 \\
$\mathrm{H}$ & 2.837 & 5.399 & 6.734 \\
$\mathrm{C}$ & 0.741 & 5.743 & 6.260 \\
$\mathrm{C}$ & 1.767 & 3.821 & 5.850
\end{tabular}




\begin{tabular}{|c|c|c|c|}
\hline H & 2.516 & 3.047 & 5.841 \\
\hline $\mathbf{N}$ & 0.520 & 3.658 & 5.438 \\
\hline & -0.127 & 4.849 & 5.685 \\
\hline $\mathrm{H}$ & -1.163 & 4.981 & 5.418 \\
\hline $\mathrm{C}$ & -4.049 & -2.752 & 3.814 \\
\hline О & -4.863 & -2.203 & 4.555 \\
\hline & -2.908 & -2.300 & 3.237 \\
\hline $\mathrm{C}$ & -2.088 & -1.100 & 3.304 \\
\hline $\mathrm{H}$ & -2.727 & -0.241 & 3.512 \\
\hline C & -1.064 & -1.259 & 4.452 \\
\hline 0 & -0.508 & -2.367 & 4.574 \\
\hline $\mathrm{O}$ & -0.927 & -0.269 & 5.245 \\
\hline $\mathrm{C}$ & -1.490 & -0.947 & 1.908 \\
\hline C & -2.386 & -0.737 & 0.897 \\
\hline $\mathrm{H}$ & -1.331 & 1.623 & 3.133 \\
\hline $\mathrm{H}$ & -3.409 & -0.435 & 1.097 \\
\hline $\mathrm{H}$ & -2.042 & -0.658 & -0.124 \\
\hline C & -0.064 & -1.279 & 1.639 \\
\hline $\mathrm{H}$ & 0.173 & -2.297 & 1.971 \\
\hline $\mathrm{H}$ & 0.149 & -1.222 & 0.571 \\
\hline $\mathrm{H}$ & 0.597 & -0.588 & 2.177 \\
\hline$S$ & -2.939 & -3.201 & 0.653 \\
\hline $\mathrm{C}$ & -2.626 & -3.486 & 2.442 \\
\hline $\mathrm{H}$ & -1.629 & -3.895 & 2.610 \\
\hline $\mathrm{C}$ & -3.795 & -4.164 & 3.237 \\
\hline $\mathrm{H}$ & -3.374 & -4.789 & 4.026 \\
\hline $\mathrm{N}$ & -4.737 & -4.956 & 2.502 \\
\hline $\mathrm{H}$ & -5.269 & -4.475 & 1.788 \\
\hline $\mathrm{C}$ & -4.264 & -6.216 & 2.168 \\
\hline $\mathrm{O}$ & -3.400 & -6.746 & 2.871 \\
\hline $\mathrm{C}$ & -4.751 & -6.854 & 0.905 \\
\hline $\mathrm{H}$ & -5.731 & -6.484 & 0.598 \\
\hline $\mathrm{H}$ & -4.830 & -7.926 & 1.104 \\
\hline $\mathrm{C}$ & -3.729 & -6.597 & -0.234 \\
\hline $\mathrm{H}$ & -3.948 & -5.624 & -0.685 \\
\hline $\mathrm{H}$ & -2.720 & -6.544 & 0.188 \\
\hline $\mathrm{C}$ & -3.755 & -7.692 & -1.299 \\
\hline $\mathrm{H}$ & -3.116 & -7.392 & -2.140 \\
\hline $\mathrm{H}$ & -3.300 & -8.597 & -0.892 \\
\hline $\mathrm{N}$ & -5.829 & -6.861 & -2.351 \\
\hline $\mathrm{H}$ & -5.339 & -6.606 & -3.219 \\
\hline $\mathrm{H}$ & -6.703 & -7.237 & -2.720 \\
\hline $\mathrm{C}$ & -5.143 & -8.049 & -1.826 \\
\hline $\mathrm{H}$ & -5.751 & -8.437 & -0.995 \\
\hline$C$ & -5.062 & -9.173 & -2.887 \\
\hline
\end{tabular}

$\begin{array}{llll}\text { C } & -5.062 & -9.173 & -2.887\end{array}$ 


$\begin{array}{lccc}\mathrm{O} & -4.076 & -9.958 & -2.814 \\ \mathrm{O} & -5.954 & -9.194 & -3.766 \\ \mathrm{Fe} & -0.382 & 1.648 & 5.120 \\ \mathrm{O} & -0.364 & 1.655 & 3.322 \\ \mathrm{C} & -3.086 & 2.655 & 4.131 \\ \mathrm{O} & -3.111 & 1.837 & 3.166 \\ \mathrm{O} & -2.205 & 2.649 & 5.055 \\ \mathrm{C} & -4.190 & 3.690 & 4.226 \\ \mathrm{H} & -5.118 & 3.146 & 4.437 \\ \mathrm{H} & -3.984 & 4.346 & 5.075 \\ \mathrm{C} & -4.404 & 4.491 & 2.934 \\ \mathrm{H} & -4.047 & 3.903 & 2.086 \\ \mathrm{H} & -3.814 & 5.408 & 2.927 \\ \mathrm{C} & -5.859 & 4.829 & 2.599 \\ \mathrm{O} & -6.784 & 4.027 & 2.923 \\ \mathrm{O} & -6.036 & 5.905 & 1.956\end{array}$

Coordinate of ${ }^{5} \mathrm{IM} 3 \mathrm{a}$

$\begin{array}{llll}\text { C } & 0.239 & 1.433 & 10.837\end{array}$

$\begin{array}{llll}\mathrm{H} & 0.914 & 0.577 & 10.856\end{array}$

$\mathrm{H} \quad-0.542 \quad 1.252 \quad 11.581$

$\begin{array}{llll}\mathrm{N} & -1.546 & 2.227 & 9.210\end{array}$

$\begin{array}{llll}\mathrm{H} & -2.167 & 2.630 & 9.917\end{array}$

$\begin{array}{llll}\text { C } & -0.336 & 1.600 & 9.466\end{array}$

$\begin{array}{llll}\text { C } & -1.715 & 2.292 & 7.872\end{array}$

$\begin{array}{llll}\mathrm{H} & -2.565 & 2.728 & 7.376\end{array}$

$\begin{array}{llll}\mathrm{N} & -0.683 & 1.730 & 7.253\end{array}$

$\begin{array}{llll}\text { C } & 0.184 & 1.300 & 8.231\end{array}$

$\begin{array}{llll}\mathrm{H} & 1.112 & 0.817 & 7.971\end{array}$

$\begin{array}{llll}\text { C } & 3.774 & -0.107 & 4.813\end{array}$

$\begin{array}{llll}\mathrm{H} & 4.601 & 0.486 & 4.405\end{array}$

$\begin{array}{llll}\mathrm{H} & 3.763 & -1.036 & 4.233\end{array}$

$\begin{array}{llll}\text { C } & 2.463 & 0.633 & 4.423\end{array}$

$\begin{array}{llll}\text { O } & 1.594 & 0.914 & 5.322\end{array}$

$\begin{array}{llll}\text { O } & 2.404 & 0.894 & 3.201\end{array}$

$\begin{array}{llll}\text { C } & 0.519 & 7.126 & 6.699\end{array}$

$\begin{array}{llll}\mathrm{H} & -0.361 & 7.552 & 6.210\end{array}$

$\begin{array}{llll}\mathrm{H} & 1.378 & 7.728 & 6.382\end{array}$

$\begin{array}{llll}\mathrm{N} & 1.907 & 5.017 & 6.338\end{array}$

$\begin{array}{llll}\mathrm{H} & 2.806 & 5.356 & 6.694\end{array}$

$\begin{array}{llll}\text { C } & 0.697 & 5.698 & 6.290\end{array}$

$\begin{array}{llll}\text { C } & 1.701 & 3.765 & 5.881\end{array}$

$\begin{array}{llll}\mathrm{H} & 2.452 & 2.996 & 5.841\end{array}$

$\begin{array}{llll}\mathrm{N} & 0.438 & 3.598 & 5.524\end{array}$

$\begin{array}{llll}\text { C } & -0.197 & 4.796 & 5.772\end{array}$ 


\begin{tabular}{|c|c|c|c|}
\hline $\mathrm{H}$ & -1.241 & 4.925 & 42 \\
\hline $\mathrm{C}$ & -4.123 & -2.763 & 3.823 \\
\hline $\mathrm{O}$ & -4.973 & -2.237 & 4.536 \\
\hline N & -2.960 & -2.292 & 3.302 \\
\hline C & -2.147 & -1.079 & 3.345 \\
\hline $\mathrm{H}$ & -2.802 & -0.224 & 3.528 \\
\hline $\mathrm{C}$ & -1.156 & -1.223 & 4.526 \\
\hline $\mathrm{O}$ & -0.573 & -2.319 & 4.641 \\
\hline $\mathrm{O}$ & -1.089 & -0.258 & 5.359 \\
\hline $\mathrm{C}$ & -1.529 & -0.971 & 1.962 \\
\hline C & -2.485 & -1.240 & 0.867 \\
\hline $\mathrm{H}$ & -1.453 & 1.571 & 3.242 \\
\hline $\mathrm{H}$ & -3.447 & -0.743 & 1.014 \\
\hline $\mathrm{H}$ & -2.081 & -0.972 & -0.109 \\
\hline C & -0.070 & -1.165 & 1.713 \\
\hline $\mathrm{H}$ & 0.222 & -2.210 & 1.907 \\
\hline $\mathrm{H}$ & 0.166 & -0.977 & 0.663 \\
\hline $\mathrm{H}$ & 0.537 & -0.491 & 2.329 \\
\hline $\mathrm{S}$ & -2.849 & -3.110 & 0.714 \\
\hline $\mathrm{C}$ & -2.630 & -3.464 & 2.522 \\
\hline $\mathrm{H}$ & -1.634 & -3.863 & 2.721 \\
\hline $\mathrm{C}$ & -3.823 & -4.168 & 3.239 \\
\hline $\mathrm{H}$ & -3.433 & -4.799 & 4.039 \\
\hline $\mathrm{N}$ & -4.716 & -4.963 & 2.451 \\
\hline $\mathrm{H}$ & -5.259 & -4.491 & 1.740 \\
\hline $\mathrm{C}$ & -4.226 & -6.219 & 2.133 \\
\hline $\mathrm{O}$ & -3.368 & -6.736 & 2.853 \\
\hline $\mathrm{C}$ & -4.692 & -6.869 & 0.868 \\
\hline $\mathrm{H}$ & -5.686 & -6.532 & 0.567 \\
\hline $\mathrm{H}$ & -4.732 & -7.943 & 1.064 \\
\hline $\mathrm{C}$ & -3.691 & -6.572 & -0.278 \\
\hline $\mathrm{H}$ & -3.930 & -5.593 & -0.706 \\
\hline $\mathrm{H}$ & -2.674 & -6.514 & 0.129 \\
\hline $\mathrm{C}$ & -3.724 & -7.650 & -1.361 \\
\hline $\mathrm{H}$ & -3.124 & -7.319 & -2.220 \\
\hline $\mathrm{H}$ & -3.236 & -8.551 & -0.988 \\
\hline $\mathrm{N}$ & -5.838 & -6.859 & -2.358 \\
\hline $\mathrm{H}$ & -5.376 & -6.608 & -3.242 \\
\hline $\mathrm{H}$ & -6.719 & -7.247 & -2.696 \\
\hline $\mathrm{C}$ & -5.121 & -8.032 & -1.846 \\
\hline $\mathrm{H}$ & -5.698 & -8.425 & -0.994 \\
\hline $\mathrm{C}$ & -5.055 & -9.167 & -2.895 \\
\hline $\mathrm{O}$ & -4.076 & -9.960 & -2.815 \\
\hline $\mathrm{O}$ & -5.952 & -9.192 & -3.769 \\
\hline $\mathrm{Fe}$ & -0.470 & 1.628 & 5.217 \\
\hline
\end{tabular}




$\begin{array}{llll}\mathrm{O} & -0.484 & 1.599 & 3.421 \\ \mathrm{C} & -3.153 & 2.677 & 4.214 \\ \mathrm{O} & -3.192 & 1.826 & 3.275 \\ \mathrm{O} & -2.289 & 2.673 & 5.150 \\ \mathrm{C} & -4.224 & 3.748 & 4.251 \\ \mathrm{H} & -5.162 & 3.245 & 4.516 \\ \mathrm{H} & -3.985 & 4.454 & 5.050 \\ \mathrm{C} & -4.436 & 4.463 & 2.912 \\ \mathrm{H} & -4.140 & 3.791 & 2.105 \\ \mathrm{H} & -3.801 & 5.347 & 2.824 \\ \mathrm{C} & -5.885 & 4.845 & 2.602 \\ \mathrm{O} & -6.823 & 4.058 & 2.930 \\ \mathrm{O} & -6.050 & 5.931 & 1.973\end{array}$

Coordinate of ${ }^{5} \mathrm{TS} 4 \mathrm{a}$

$\begin{array}{llll}\text { C } & 0.137 & 1.438 & 10.900\end{array}$

$\begin{array}{llll}\mathrm{H} & 0.797 & 0.569 & 10.918\end{array}$

$\begin{array}{llll}\mathrm{H} & -0.637 & 1.275 & 11.656\end{array}$

$\begin{array}{llll}\mathrm{N} & -1.634 & 2.271 & 9.269\end{array}$

$\begin{array}{llll}\mathrm{H} & -2.238 & 2.709 & 9.969\end{array}$

$\begin{array}{llll}\text { C } & -0.452 & 1.598 & 9.535\end{array}$

$\begin{array}{llll}\text { C } & -1.807 & 2.300 & 7.927\end{array}$

$\begin{array}{llll}\mathrm{H} & -2.641 & 2.760 & 7.423\end{array}$

$\begin{array}{llll}\mathrm{N} & -0.816 & 1.668 & 7.312\end{array}$

$\begin{array}{llll}\text { C } & 0.035 & 1.234 & 8.305\end{array}$

$\begin{array}{llll}\mathrm{H} & 0.947 & 0.706 & 8.073\end{array}$

$\begin{array}{llll}\text { C } & 3.902 & 0.036 & 4.778\end{array}$

$\begin{array}{llll}\mathrm{H} & 4.715 & 0.710 & 4.485\end{array}$

$\begin{array}{llll}\mathrm{H} & 4.009 & -0.837 & 4.125\end{array}$

$\begin{array}{llll}\text { C } & 2.573 & 0.710 & 4.353\end{array}$

$\begin{array}{llll}\text { O } & 1.647 & 0.916 & 5.208\end{array}$

$\begin{array}{llll}\text { O } & 2.533 & 0.994 & 3.132\end{array}$

$\begin{array}{llll}\text { C } & 0.498 & 7.092 & 6.706\end{array}$

$\begin{array}{llll}\mathrm{H} & -0.392 & 7.500 & 6.219\end{array}$

$\begin{array}{llll}\mathrm{H} & 1.344 & 7.707 & 6.378\end{array}$

$\begin{array}{llll}\mathrm{N} & 1.925 & 5.009 & 6.363\end{array}$

$\begin{array}{llll}\mathrm{H} & 2.813 & 5.361 & 6.730\end{array}$

$\begin{array}{llll}\text { C } & 0.701 & 5.665 & 6.303\end{array}$

$\begin{array}{llll}\text { C } & 1.747 & 3.757 & 5.892\end{array}$

$\begin{array}{llll}\mathrm{H} & 2.524 & 3.013 & 5.857\end{array}$

$\begin{array}{llll}\mathrm{N} & 0.495 & 3.562 & 5.516\end{array}$

$\begin{array}{llll}\text { C } & -0.166 & 4.747 & 5.766\end{array}$

$\begin{array}{llll}\mathrm{H} & -1.211 & 4.853 & 5.526\end{array}$

$\begin{array}{llll}\text { C } & -3.690 & -2.401 & 3.557\end{array}$

$\begin{array}{llll}\text { O } & -4.420 & -1.875 & 4.383\end{array}$ 


\begin{tabular}{|c|c|c|c|}
\hline $\mathrm{V}$ & -2.562 & -1.987 & 2 \\
\hline $\mathrm{C}$ & -1.372 & -1.140 & 2.992 \\
\hline $\mathrm{H}$ & -1.434 & 0.043 & 3.039 \\
\hline $\mathrm{C}$ & -0.746 & -1.451 & 4.389 \\
\hline $\mathrm{O}$ & -0.344 & -2.627 & 4.563 \\
\hline $\mathrm{O}$ & -0.830 & -0.545 & 5.262 \\
\hline $\mathrm{C}$ & -0.641 & -1.324 & 1.743 \\
\hline $\mathrm{C}$ & -1.450 & -1.433 & 0.497 \\
\hline $\mathrm{H}$ & -1.781 & 1.695 & 3.202 \\
\hline $\mathrm{H}$ & -2.191 & -0.629 & 0.453 \\
\hline $\mathrm{H}$ & -0.825 & -1.402 & -0.395 \\
\hline $\mathrm{C}$ & 0.834 & -1.396 & 1.690 \\
\hline $\mathrm{H}$ & 1.147 & -2.407 & 1.999 \\
\hline $\mathrm{H}$ & 1.220 & -1.250 & 0.681 \\
\hline $\mathrm{H}$ & 1.300 & -0.645 & 2.342 \\
\hline S & -2.445 & -3.045 & 0.398 \\
\hline $\mathrm{C}$ & -2.335 & -3.268 & 2.210 \\
\hline $\mathrm{H}$ & -1.370 & -3.699 & 2.487 \\
\hline $\mathrm{C}$ & -3.537 & -3.834 & 2.987 \\
\hline $\mathrm{H}$ & -3.157 & -4.460 & 3.798 \\
\hline $\mathrm{N}$ & -4.505 & -4.621 & 2.278 \\
\hline $\mathrm{H}$ & -5.006 & -4.185 & 1.515 \\
\hline $\mathrm{C}$ & -4.146 & -5.952 & 2.117 \\
\hline $\mathrm{O}$ & -3.357 & -6.469 & 2.912 \\
\hline $\mathrm{C}$ & -4.641 & -6.687 & 0.909 \\
\hline $\mathrm{H}$ & -5.615 & -6.335 & 0.564 \\
\hline $\mathrm{H}$ & -4.729 & -7.739 & 1.194 \\
\hline $\mathrm{C}$ & -3.615 & -6.524 & -0.244 \\
\hline $\mathrm{H}$ & -3.807 & -5.569 & -0.744 \\
\hline $\mathrm{H}$ & -2.601 & -6.483 & 0.170 \\
\hline $\mathrm{C}$ & -3.680 & -7.667 & -1.261 \\
\hline $\mathrm{H}$ & -3.057 & -7.408 & -2.128 \\
\hline $\mathrm{H}$ & -3.226 & -8.562 & -0.830 \\
\hline $\mathrm{N}$ & -5.797 & -6.823 & -2.207 \\
\hline $\mathrm{H}$ & -5.385 & -6.587 & -3.119 \\
\hline $\mathrm{H}$ & -6.714 & -7.179 & -2.478 \\
\hline $\mathrm{C}$ & -5.087 & -8.022 & -1.741 \\
\hline $\mathrm{H}$ & -5.656 & -8.428 & -0.891 \\
\hline $\mathrm{C}$ & -5.051 & -9.128 & -2.829 \\
\hline $\mathrm{O}$ & -4.075 & -9.929 & -2.797 \\
\hline $\mathrm{O}$ & -5.967 & -9.117 & -3.682 \\
\hline $\mathrm{Fe}$ & -0.413 & 1.545 & 5.154 \\
\hline $\mathrm{O}$ & -0.820 & 1.412 & 3.246 \\
\hline $\mathrm{C}$ & -3.229 & 2.773 & 4.238 \\
\hline $\mathrm{O}$ & -3.304 & 1.976 & 3.244 \\
\hline
\end{tabular}




$\begin{array}{llll}\mathrm{O} & -2.355 & 2.690 & 5.148 \\ \mathrm{C} & -4.269 & 3.868 & 4.308 \\ \mathrm{H} & -5.211 & 3.388 & 4.599 \\ \mathrm{H} & -3.991 & 4.569 & 5.098 \\ \mathrm{C} & -4.494 & 4.579 & 2.966 \\ \mathrm{H} & -4.154 & 3.919 & 2.164 \\ \mathrm{H} & -3.897 & 5.488 & 2.886 \\ \mathrm{C} & -5.948 & 4.901 & 2.630 \\ \mathrm{O} & -6.862 & 4.081 & 2.946 \\ \mathrm{O} & -6.146 & 5.978 & 1.994\end{array}$

Coordinate of ${ }^{5} \mathrm{~Pa}$

$\begin{array}{lrrr}\mathrm{C} & 0.211 & 1.473 & 11.077 \\ \mathrm{H} & 0.877 & 0.611 & 11.115 \\ \mathrm{H} & -0.525 & 1.350 & 11.877 \\ \mathrm{~N} & -1.661 & 2.147 & 9.477 \\ \mathrm{H} & -2.262 & 2.599 & 10.176 \\ \mathrm{C} & -0.438 & 1.549 & 9.734 \\ \mathrm{C} & -1.869 & 2.117 & 8.141 \\ \mathrm{H} & -2.733 & 2.528 & 7.642 \\ \mathrm{~N} & -0.860 & 1.515 & 7.525 \\ \mathrm{C} & 0.037 & 1.165 & 8.507 \\ \mathrm{H} & 0.972 & 0.684 & 8.265 \\ \mathrm{C} & 3.806 & -0.135 & 4.756 \\ \mathrm{H} & 4.612 & 0.479 & 4.338 \\ \mathrm{H} & 3.796 & -1.055 & 4.159 \\ \mathrm{C} & 2.459 & 0.571 & 4.474 \\ \mathrm{O} & 1.660 & 0.807 & 5.424 \\ \mathrm{O} & 2.250 & 0.845 & 3.248 \\ \mathrm{C} & 0.553 & 7.121 & 6.739 \\ \mathrm{H} & -0.330 & 7.517 & 6.231 \\ \mathrm{H} & 1.408 & 7.718 & 6.402 \\ \mathrm{~N} & 1.963 & 5.012 & 6.464 \\ \mathrm{H} & 2.865 & 5.374 & 6.788 \\ \mathrm{C} & 0.746 & 5.679 & 6.394 \\ \mathrm{C} & 1.760 & 3.733 & 6.090 \\ \mathrm{H} & 2.522 & 2.972 & 6.079 \\ \mathrm{~N} & 0.495 & 3.535 & 5.758 \\ \mathrm{C} & -0.146 & 4.740 & 5.944 \\ \mathrm{H} & -1.196 & 4.843 & 5.719 \\ \mathrm{C} & -4.342 & -3.025 & 3.892 \\ \mathrm{O} & -5.095 & -2.440 & 4.659 \\ \mathrm{~N} & -3.208 & -2.611 & 3.255 \\ \mathrm{C} & -2.229 & -1.592 & 3.236 \\ \mathrm{H} & 0.515 & 1.299 & 3.110 \\ & & & \end{array}$




\begin{tabular}{|c|c|c|c|}
\hline $\mathrm{C}$ & -1.476 & -1.427 & 4.535 \\
\hline $\mathrm{O}$ & -1.423 & -2.447 & 5.255 \\
\hline $\mathrm{O}$ & -0.900 & -0.320 & 4.751 \\
\hline $\mathrm{C}$ & -1.850 & -1.268 & 1.980 \\
\hline $\mathrm{C}$ & -2.776 & -1.662 & 0.855 \\
\hline $\mathrm{H}$ & -1.131 & 1.455 & 3.124 \\
\hline $\mathrm{H}$ & -3.741 & -1.164 & 0.972 \\
\hline $\mathrm{H}$ & -2.352 & -1.382 & -0.111 \\
\hline $\mathrm{C}$ & -0.536 & -0.684 & 1.572 \\
\hline $\mathrm{H}$ & -0.075 & -1.352 & 0.833 \\
\hline $\mathrm{H}$ & -0.678 & 0.286 & 1.082 \\
\hline $\mathrm{H}$ & 0.159 & -0.579 & 2.402 \\
\hline $\mathrm{S}$ & -3.106 & -3.495 & 0.721 \\
\hline $\mathrm{C}$ & -2.958 & -3.839 & 2.526 \\
\hline $\mathrm{H}$ & -1.980 & -4.274 & 2.743 \\
\hline $\mathrm{C}$ & -4.160 & -4.456 & 3.303 \\
\hline $\mathrm{H}$ & -3.785 & -5.122 & 4.084 \\
\hline $\mathrm{N}$ & -5.129 & -5.189 & 2.542 \\
\hline $\mathrm{H}$ & -5.560 & -4.677 & 1.782 \\
\hline $\mathrm{C}$ & -4.716 & -6.480 & 2.217 \\
\hline $\mathrm{O}$ & -3.987 & -7.105 & 2.983 \\
\hline $\mathrm{C}$ & -5.076 & -7.014 & 0.866 \\
\hline $\mathrm{H}$ & -6.034 & -6.638 & 0.501 \\
\hline $\mathrm{H}$ & -5.140 & -8.096 & 0.974 \\
\hline $\mathrm{C}$ & -3.977 & -6.665 & -0.171 \\
\hline $\mathrm{H}$ & -4.202 & -5.690 & -0.610 \\
\hline $\mathrm{H}$ & -3.002 & -6.592 & 0.326 \\
\hline $\mathrm{C}$ & -3.890 & -7.722 & -1.272 \\
\hline $\mathrm{H}$ & -3.200 & -7.381 & -2.055 \\
\hline $\mathrm{H}$ & -3.446 & -8.632 & -0.862 \\
\hline $\mathrm{N}$ & -5.881 & -6.907 & -2.490 \\
\hline $\mathrm{H}$ & -5.326 & -6.645 & -3.317 \\
\hline $\mathrm{H}$ & -6.714 & -7.293 & -2.937 \\
\hline $\mathrm{C}$ & -5.231 & -8.086 & -1.904 \\
\hline $\mathrm{H}$ & -5.903 & -8.469 & -1.121 \\
\hline $\mathrm{C}$ & -5.075 & -9.216 & -2.943 \\
\hline $\mathrm{O}$ & -4.069 & -9.970 & -2.824 \\
\hline $\mathrm{O}$ & -5.937 & -9.276 & -3.851 \\
\hline $\mathrm{Fe}$ & -0.467 & 1.590 & 5.418 \\
\hline $\mathrm{O}$ & -0.257 & 1.894 & 3.267 \\
\hline $\mathrm{C}$ & -3.060 & 2.574 & 4.134 \\
\hline $\mathrm{O}$ & -2.917 & 1.710 & 3.214 \\
\hline $\mathrm{O}$ & -2.333 & 2.658 & 5.170 \\
\hline $\mathrm{C}$ & -4.212 & 3.580 & 4.032 \\
\hline $\mathrm{H}$ & -5.042 & 3.177 & 4.627 \\
\hline
\end{tabular}




$\begin{array}{llll}\mathrm{H} & -3.894 & 4.503 & 4.527 \\ \mathrm{C} & -4.688 & 3.843 & 2.602 \\ \mathrm{H} & -4.824 & 2.867 & 2.125 \\ \mathrm{H} & -3.927 & 4.396 & 2.045 \\ \mathrm{C} & -6.023 & 4.578 & 2.494 \\ \mathrm{O} & -7.070 & 3.954 & 2.860 \\ \mathrm{O} & -6.015 & 5.749 & 2.013\end{array}$

Coordinate of ${ }^{5} \mathrm{TS} 4 \mathrm{a}-\mathrm{reb}$

\begin{tabular}{|c|c|c|c|}
\hline$r$ & 0.206 & 1.442 & 10.826 \\
\hline $\mathrm{H}$ & 0.869 & 0.575 & 10.844 \\
\hline $\mathrm{H}$ & -0.579 & 1.269 & 11.568 \\
\hline & -1.546 & 2.292 & 9.188 \\
\hline & -2.167 & 2.700 & 9.892 \\
\hline$C$ & -0.358 & 1.627 & 9.452 \\
\hline $\mathrm{C}$ & -1.692 & 2.376 & 7.846 \\
\hline ח & -2.529 & 2.833 & 7.346 \\
\hline$\Lambda$ & -0.669 & 1.792 & 7.234 \\
\hline $\mathrm{C}$ & 0.168 & 1.325 & 8.220 \\
\hline $\mathrm{H}$ & 1.085 & 0.816 & 7.971 \\
\hline $\mathrm{C}$ & 3.795 & -0.125 & 4.777 \\
\hline $\mathrm{H}$ & 4.637 & 0.451 & 4.376 \\
\hline $\mathrm{H}$ & 3.774 & -1.056 & 4.200 \\
\hline $\mathrm{C}$ & 2.503 & 0.638 & 4.372 \\
\hline $\mathrm{O}$ & 1.638 & 0.957 & 5.257 \\
\hline $\mathrm{O}$ & 2.449 & 0.885 & 3.145 \\
\hline $\mathrm{C}$ & 0.507 & 7.127 & 6.681 \\
\hline $\mathrm{H}$ & -0.376 & 7.560 & 6.203 \\
\hline $\mathrm{H}$ & 1.364 & 7.735 & 6.365 \\
\hline $\mathrm{N}$ & 1.896 & 5.026 & 6.291 \\
\hline $\mathrm{H}$ & 2.790 & 5.357 & 6.666 \\
\hline $\mathrm{C}$ & 0.685 & 5.707 & 6.245 \\
\hline $\mathrm{C}$ & 1.699 & 3.791 & 5.785 \\
\hline $\mathrm{H}$ & 2.452 & 3.024 & 5.738 \\
\hline $\mathrm{N}$ & 0.442 & 3.632 & 5.403 \\
\hline $\mathrm{C}$ & -0.198 & 4.821 & 5.683 \\
\hline $\mathrm{H}$ & -1.240 & 4.957 & 5.443 \\
\hline $\mathrm{C}$ & -4.024 & -2.725 & 3.805 \\
\hline $\mathrm{O}$ & -4.886 & -2.248 & 4.534 \\
\hline $\mathrm{N}$ & -2.846 & -2.224 & 3.345 \\
\hline 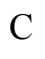 & -2.027 & -1.028 & 3.499 \\
\hline $\mathrm{H}$ & -2.691 & -0.193 & 3.722 \\
\hline $\mathrm{C}$ & -1.057 & -1.256 & 4.674 \\
\hline $\mathrm{O}$ & -0.537 & -2.388 & 4.761 \\
\hline 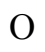 & -0.929 & -0.307 & 5.509 \\
\hline
\end{tabular}




\begin{tabular}{|c|c|c|c|}
\hline $\mathrm{C}$ & -1.382 & -0.814 & 142 \\
\hline $\mathrm{C}$ & -2.357 & -0.915 & 1.036 \\
\hline $\mathrm{H}$ & -1.610 & 1.451 & 3.197 \\
\hline $\mathrm{H}$ & -3.302 & -0.414 & 1.248 \\
\hline $\mathrm{H}$ & -1.952 & -0.558 & 0.091 \\
\hline $\mathrm{C}$ & 0.038 & -1.167 & 1.846 \\
\hline $\mathrm{H}$ & 0.204 & -2.249 & 1.975 \\
\hline $\mathrm{H}$ & 0.271 & -0.954 & 0.801 \\
\hline $\mathrm{H}$ & 0.731 & -0.597 & 2.472 \\
\hline S & -2.748 & -2.763 & 0.701 \\
\hline $\mathrm{C}$ & -2.524 & -3.315 & 2.459 \\
\hline $\mathrm{H}$ & -1.531 & -3.739 & 2.609 \\
\hline $\mathrm{C}$ & -3.710 & -4.081 & 3.120 \\
\hline $\mathrm{H}$ & -3.302 & -4.753 & 3.878 \\
\hline N & -4.587 & -4.859 & 2.305 \\
\hline $\mathrm{H}$ & -5.098 & -4.389 & 1.569 \\
\hline $\mathrm{C}$ & -4.159 & -6.152 & 2.056 \\
\hline $\mathrm{O}$ & -3.359 & -6.690 & 2.825 \\
\hline $\mathrm{C}$ & -4.622 & -6.817 & 0.800 \\
\hline $\mathrm{H}$ & -5.618 & -6.487 & 0.496 \\
\hline $\mathrm{H}$ & -4.657 & -7.889 & 1.008 \\
\hline $\mathrm{C}$ & -3.627 & -6.528 & -0.351 \\
\hline $\mathrm{H}$ & -3.855 & -5.544 & -0.776 \\
\hline $\mathrm{H}$ & -2.606 & -6.485 & 0.047 \\
\hline $\mathrm{C}$ & -3.684 & -7.608 & -1.433 \\
\hline $\mathrm{H}$ & -3.121 & -7.273 & -2.315 \\
\hline $\mathrm{H}$ & -3.174 & -8.504 & -1.076 \\
\hline $\mathrm{N}$ & -5.843 & -6.848 & -2.354 \\
\hline $\mathrm{H}$ & -5.414 & -6.599 & -3.256 \\
\hline $\mathrm{H}$ & -6.734 & -7.245 & -2.653 \\
\hline $\mathrm{C}$ & -5.094 & -8.010 & -1.864 \\
\hline $\mathrm{H}$ & -5.633 & -8.406 & -0.989 \\
\hline $\mathrm{C}$ & -5.050 & -9.156 & -2.901 \\
\hline $\mathrm{O}$ & -4.079 & -9.960 & -2.815 \\
\hline $\mathrm{O}$ & -5.952 & -9.180 & -3.769 \\
\hline $\mathrm{Fe}$ & -0.455 & 1.624 & 5.150 \\
\hline $\mathrm{O}$ & -0.636 & 1.320 & 3.304 \\
\hline $\mathrm{C}$ & -3.185 & 2.678 & 4.161 \\
\hline $\mathrm{O}$ & -3.271 & 1.824 & 3.223 \\
\hline $\mathrm{O}$ & -2.284 & 2.669 & 5.057 \\
\hline $\mathrm{C}$ & -4.243 & 3.756 & 4.233 \\
\hline $\mathrm{H}$ & -5.185 & 3.258 & 4.490 \\
\hline $\mathrm{H}$ & -3.989 & 4.441 & 5.045 \\
\hline $\mathrm{C}$ & -4.452 & 4.504 & 2.910 \\
\hline $\mathrm{H}$ & -4.127 & 3.867 & 2.086 \\
\hline
\end{tabular}

$\begin{array}{llll}\mathrm{H} & -4.127 & 3.867 & 2.086\end{array}$ 


$\begin{array}{llll}\mathrm{H} & -3.838 & 5.403 & 2.854 \\ \mathrm{C} & -5.904 & 4.863 & 2.590 \\ \mathrm{O} & -6.834 & 4.065 & 2.915 \\ \mathrm{O} & -6.077 & 5.946 & 1.959\end{array}$

Coordinate of ${ }^{5} \mathrm{~Pa}$-reb

$\begin{array}{llll}\text { C } & 0.135 & 1.491 & 10.913\end{array}$

$\begin{array}{llll}\mathrm{H} & 0.780 & 0.611 & 10.930\end{array}$

$\begin{array}{llll}\mathrm{H} & -0.642 & 1.342 & 11.669\end{array}$

$\begin{array}{llll}\mathrm{N} & -1.604 & 2.374 & 9.265\end{array}$

$\begin{array}{llll}\mathrm{H} & -2.218 & 2.805 & 9.963\end{array}$

$\begin{array}{llll}\text { C } & -0.443 & 1.672 & 9.545\end{array}$

$\begin{array}{llll}\text { C } & -1.738 & 2.440 & 7.917\end{array}$

$\begin{array}{llll}\mathrm{H} & -2.559 & 2.924 & 7.414\end{array}$

$\begin{array}{llll}\mathrm{N} & -0.742 & 1.806 & 7.316\end{array}$

$\begin{array}{llll}\text { C } & 0.069 & 1.327 & 8.320\end{array}$

$\begin{array}{llll}\mathrm{H} & 0.973 & 0.780 & 8.098\end{array}$

$\begin{array}{llll}\text { C } & 3.957 & 0.065 & 4.708\end{array}$

$\begin{array}{llll}\mathrm{H} & 4.807 & 0.679 & 4.388\end{array}$

$\begin{array}{llll}\mathrm{H} & 3.982 & -0.828 & 4.073\end{array}$

$\begin{array}{llll}\text { C } & 2.662 & 0.813 & 4.306\end{array}$

$\begin{array}{llll}\mathrm{O} & 1.771 & 1.046 & 5.201\end{array}$

$\begin{array}{llll}\text { O } & 2.581 & 1.135 & 3.105\end{array}$

$\begin{array}{llll}\text { C } & 0.548 & 7.177 & 6.745\end{array}$

$\begin{array}{llll}\mathrm{H} & -0.343 & 7.580 & 6.257\end{array}$

$\begin{array}{llll}\mathrm{H} & 1.388 & 7.811 & 6.438\end{array}$

$\begin{array}{llll}\mathrm{N} & 1.997 & 5.106 & 6.423\end{array}$

$\begin{array}{llll}\mathrm{H} & 2.869 & 5.441 & 6.842\end{array}$

$\begin{array}{llll}\text { C } & 0.778 & 5.765 & 6.311\end{array}$

$\begin{array}{llll}\text { C } & 1.861 & 3.879 & 5.886\end{array}$

$\begin{array}{llll}\mathrm{H} & 2.639 & 3.135 & 5.873\end{array}$

$\begin{array}{llll}\mathrm{N} & 0.636 & 3.700 & 5.413\end{array}$

$\begin{array}{llll}\text { C } & -0.046 & 4.872 & 5.673\end{array}$

$\begin{array}{llll}\mathrm{H} & -1.071 & 4.995 & 5.361\end{array}$

$\begin{array}{llll}\text { C } & -4.085 & -2.790 & 3.730\end{array}$

$\begin{array}{llll}\text { O } & -4.969 & -2.304 & 4.432\end{array}$

$\begin{array}{llll}\mathrm{N} & -2.896 & -2.293 & 3.307\end{array}$

$\begin{array}{llll}\text { C } & -2.079 & -1.139 & 3.548\end{array}$

$\begin{array}{llll}\mathrm{H} & -2.726 & -0.305 & 3.820\end{array}$

$\begin{array}{llll}\text { C } & -1.082 & -1.426 & 4.673\end{array}$

$\begin{array}{llll}\mathrm{O} & -0.826 & -2.631 & 4.915\end{array}$

$\begin{array}{llll}\mathrm{O} & -0.548 & -0.429 & 5.236\end{array}$

$\begin{array}{llll}\text { C } & -1.339 & -0.752 & 2.208\end{array}$

$\begin{array}{llll}\text { C } & -2.296 & -1.053 & 1.024\end{array}$

$\begin{array}{llll}\mathrm{H} & -1.949 & 1.071 & 2.590\end{array}$ 


\begin{tabular}{|c|c|c|c|}
\hline $\mathrm{H}$ & -3.244 & -0.528 & 1.176 \\
\hline $\mathrm{H}$ & -1.838 & -0.676 & 0.109 \\
\hline C & 0.031 & -1.405 & 2.001 \\
\hline $\mathrm{H}$ & 0.026 & -2.487 & 2.127 \\
\hline $\mathrm{H}$ & 0.383 & -1.190 & 0.988 \\
\hline $\mathrm{H}$ & 0.749 & -0.970 & 2.697 \\
\hline S & -2.644 & -2.848 & 0.695 \\
\hline C & -2.528 & -3.396 & 2.459 \\
\hline $\mathrm{H}$ & -1.550 & -3.825 & 2.675 \\
\hline $\mathrm{C}$ & -3.747 & -4.148 & 3.067 \\
\hline $\mathrm{H}$ & -3.381 & -4.815 & 3.851 \\
\hline $\mathrm{N}$ & -4.572 & -4.933 & 2.210 \\
\hline $\mathrm{H}$ & -5.006 & -4.473 & 1.418 \\
\hline $\mathrm{C}$ & -4.143 & -6.236 & 2.032 \\
\hline $\mathrm{O}$ & -3.420 & -6.766 & 2.879 \\
\hline $\mathrm{C}$ & -4.514 & -6.920 & 0.761 \\
\hline $\mathrm{H}$ & -5.513 & -6.639 & 0.425 \\
\hline $\mathrm{H}$ & -4.497 & -7.992 & 0.968 \\
\hline $\mathrm{C}$ & -3.518 & -6.570 & -0.368 \\
\hline $\mathrm{H}$ & -3.734 & -5.556 & -0.724 \\
\hline $\mathrm{H}$ & -2.494 & -6.569 & 0.024 \\
\hline $\mathrm{C}$ & -3.614 & -7.577 & -1.520 \\
\hline $\mathrm{H}$ & -3.163 & -7.145 & -2.423 \\
\hline $\mathrm{H}$ & -3.029 & -8.466 & -1.284 \\
\hline $\mathrm{N}$ & -5.879 & -6.876 & -2.183 \\
\hline $\mathrm{H}$ & -5.672 & -6.675 & -3.169 \\
\hline $\mathrm{H}$ & -6.835 & -7.233 & -2.197 \\
\hline $\mathrm{C}$ & -5.041 & -8.028 & -1.836 \\
\hline $\mathrm{H}$ & -5.468 & -8.488 & -0.934 \\
\hline $\mathrm{C}$ & -5.042 & -9.142 & -2.904 \\
\hline $\mathrm{O}$ & -4.088 & -9.970 & -2.829 \\
\hline $\mathrm{O}$ & -5.952 & -9.132 & -3.762 \\
\hline $\mathrm{Fe}$ & -0.227 & 1.615 & 5.201 \\
\hline $\mathrm{O}$ & -1.107 & 0.644 & 2.262 \\
\hline $\mathrm{C}$ & -3.042 & 2.600 & 3.977 \\
\hline $\mathrm{O}$ & -3.309 & 1.746 & 3.069 \\
\hline $\mathrm{O}$ & -2.008 & 2.570 & 4.706 \\
\hline $\mathrm{C}$ & -4.064 & 3.702 & 4.186 \\
\hline $\mathrm{H}$ & -5.008 & 3.211 & 4.449 \\
\hline $\mathrm{H}$ & -3.756 & 4.329 & 5.027 \\
\hline $\mathrm{C}$ & -4.319 & 4.546 & 2.923 \\
\hline $\mathrm{H}$ & -3.933 & 4.019 & 2.047 \\
\hline $\mathrm{H}$ & -3.773 & 5.488 & 2.953 \\
\hline $\mathrm{C}$ & -5.787 & 4.838 & 2.589 \\
\hline $\mathrm{O}$ & -6.692 & 4.020 & 2.925 \\
\hline
\end{tabular}


$\begin{array}{llll}\text { O } & -5.990 & 5.900 & 1.929\end{array}$

\begin{tabular}{|c|c|c|c|}
\hline \multicolumn{4}{|c|}{ Coordinate of ${ }^{3} \mathrm{R}$} \\
\hline $\mathrm{C}$ & 0.159 & 1.465 & 10.772 \\
\hline $\mathrm{H}$ & 0.817 & 0.594 & 10.796 \\
\hline $\mathrm{H}$ & -0.639 & 1.290 & 11.500 \\
\hline $\mathrm{N}$ & -1.582 & 2.293 & 9.103 \\
\hline $\mathrm{H}$ & -2.208 & 2.709 & 9.797 \\
\hline $\mathrm{C}$ & -0.386 & 1.651 & 9.391 \\
\hline $\mathrm{C}$ & -1.724 & 2.337 & 7.758 \\
\hline $\mathrm{H}$ & -2.561 & 2.774 & 7.239 \\
\hline $\mathrm{N}$ & -0.694 & 1.747 & 7.166 \\
\hline $\mathrm{C}$ & 0.148 & 1.324 & 8.170 \\
\hline $\mathrm{H}$ & 1.079 & 0.833 & 7.941 \\
\hline $\mathrm{C}$ & 3.786 & 0.003 & 4.816 \\
\hline $\mathrm{H}$ & 4.516 & 0.759 & 4.499 \\
\hline $\mathrm{H}$ & 3.939 & -0.853 & 4.154 \\
\hline $\mathrm{C}$ & 2.366 & 0.536 & 4.486 \\
\hline $\mathrm{O}$ & 1.608 & 0.955 & 5.432 \\
\hline $\mathrm{O}$ & 2.087 & 0.537 & 3.268 \\
\hline $\mathrm{C}$ & 0.559 & 7.147 & 6.704 \\
\hline $\mathrm{H}$ & -0.324 & 7.555 & 6.206 \\
\hline $\mathrm{H}$ & 1.409 & 7.768 & 6.398 \\
\hline $\mathrm{N}$ & 1.991 & 5.060 & 6.388 \\
\hline $\mathrm{H}$ & 2.871 & 5.401 & 6.785 \\
\hline $\mathrm{C}$ & 0.774 & 5.725 & 6.293 \\
\hline $\mathrm{C}$ & 1.819 & 3.809 & 5.909 \\
\hline $\mathrm{H}$ & 2.580 & 3.048 & 5.917 \\
\hline $\mathrm{N}$ & 0.578 & 3.630 & 5.490 \\
\hline $\mathrm{C}$ & -0.081 & 4.817 & 5.721 \\
\hline $\mathrm{H}$ & -1.116 & 4.936 & 5.442 \\
\hline $\mathrm{C}$ & -4.347 & -2.805 & 4.036 \\
\hline $\mathrm{O}$ & -5.295 & -2.150 & 4.462 \\
\hline $\mathrm{N}$ & -3.044 & -2.496 & 3.817 \\
\hline $\mathrm{C}$ & -2.225 & -1.354 & 3.449 \\
\hline $\mathrm{H}$ & -2.789 & -0.425 & 3.514 \\
\hline $\mathrm{C}$ & -1.087 & -1.331 & 4.502 \\
\hline $\mathrm{O}$ & -0.419 & -2.378 & 4.638 \\
\hline $\mathrm{O}$ & -1.018 & -0.302 & 5.250 \\
\hline $\mathrm{C}$ & -1.798 & -1.615 & 1.939 \\
\hline $\mathrm{C}$ & -2.538 & -0.645 & 1.031 \\
\hline $\mathrm{H}$ & -2.168 & 0.368 & 1.196 \\
\hline $\mathrm{H}$ & -3.617 & -0.652 & 1.211 \\
\hline $\mathrm{H}$ & -2.362 & -0.910 & -0.015 \\
\hline
\end{tabular}




\begin{tabular}{|c|c|c|c|}
\hline$C_{1}$ & -0.291 & -1.505 & 1.685 \\
\hline $\mathrm{H}$ & 0.238 & -2.356 & 2.110 \\
\hline $\mathrm{H}$ & -0.102 & -1.505 & 0.607 \\
\hline $\mathrm{H}$ & 0.099 & -0.570 & 2.102 \\
\hline S & -2.380 & -3.363 & 1.490 \\
\hline $\mathrm{C}$ & -2.676 & -3.780 & 3.244 \\
\hline $\mathrm{H}$ & -1.813 & -4.252 & 3.707 \\
\hline $\mathrm{C}$ & -4.088 & -4.287 & 3.654 \\
\hline $\mathrm{H}$ & -4.024 & -4.906 & 4.551 \\
\hline $\mathrm{N}$ & -4.852 & -4.967 & 2.641 \\
\hline $\mathrm{H}$ & -5.467 & -4.441 & 2.038 \\
\hline $\mathrm{C}$ & -4.282 & -6.134 & 2.187 \\
\hline $\mathrm{O}$ & -3.369 & -6.641 & 2.850 \\
\hline $\mathrm{C}$ & -4.742 & -6.736 & 0.892 \\
\hline $\mathrm{H}$ & -5.704 & -6.342 & 0.560 \\
\hline $\mathrm{H}$ & -4.867 & -7.806 & 1.089 \\
\hline $\mathrm{C}$ & -3.688 & -6.532 & -0.225 \\
\hline $\mathrm{H}$ & -3.863 & -5.558 & -0.694 \\
\hline $\mathrm{H}$ & -2.688 & -6.504 & 0.221 \\
\hline $\mathrm{C}$ & -3.724 & -7.647 & -1.276 \\
\hline $\mathrm{H}$ & -3.089 & -7.362 & -2.125 \\
\hline $\mathrm{H}$ & -3.270 & -8.548 & -0.859 \\
\hline $\mathrm{N}$ & -5.813 & -6.828 & -2.312 \\
\hline $\mathrm{H}$ & -5.337 & -6.579 & -3.189 \\
\hline $\mathrm{H}$ & -6.691 & -7.210 & -2.666 \\
\hline $\mathrm{C}$ & -5.116 & -8.013 & -1.795 \\
\hline $\mathrm{H}$ & -5.714 & -8.403 & -0.957 \\
\hline $\mathrm{C}$ & -5.053 & -9.140 & -2.857 \\
\hline $\mathrm{O}$ & -4.073 & -9.934 & -2.799 \\
\hline $\mathrm{O}$ & -5.959 & -9.156 & -3.722 \\
\hline $\mathrm{Fe}$ & -0.341 & 1.592 & 5.043 \\
\hline $\mathrm{O}$ & -0.128 & 1.555 & 3.403 \\
\hline $\mathrm{C}$ & -3.011 & 2.575 & 4.039 \\
\hline $\mathrm{O}$ & -3.101 & 1.736 & 3.112 \\
\hline $\mathrm{O}$ & -2.082 & 2.623 & 4.924 \\
\hline $\mathrm{C}$ & -4.116 & 3.620 & 4.181 \\
\hline $\mathrm{H}$ & -5.047 & 3.071 & 4.362 \\
\hline $\mathrm{H}$ & -3.915 & 4.240 & 5.059 \\
\hline $\mathrm{C}$ & -4.325 & 4.487 & 2.930 \\
\hline $\mathrm{H}$ & -3.926 & 3.965 & 2.057 \\
\hline $\mathrm{H}$ & -3.765 & 5.421 & 2.989 \\
\hline $\mathrm{C}$ & -5.781 & 4.814 & 2.570 \\
\hline $\mathrm{O}$ & -6.710 & 4.011 & 2.880 \\
\hline $\mathrm{O}$ & -5.955 & 5.889 & 1.922 \\
\hline
\end{tabular}




\begin{tabular}{|c|c|c|c|}
\hline \multicolumn{3}{|c|}{ Coordinate of ${ }^{3} \mathrm{TS} 1 \mathrm{a}$} & \multirow[b]{2}{*}{10.632} \\
\hline $\mathrm{C}$ & 0.148 & 1.450 & \\
\hline$\Pi$ & 0.804 & 0.578 & 10.642 \\
\hline I & -0.666 & 1.254 & 11.338 \\
\hline $\mathrm{N}$ & -1.553 & 2.359 & 8.972 \\
\hline $\mathrm{H}$ & -2.175 & 2.765 & 9.672 \\
\hline C & -0.370 & 1.687 & 9.246 \\
\hline $\mathrm{C}$ & -1.688 & 2.441 & 7.627 \\
\hline $\mathrm{H}$ & -2.521 & 2.901 & 7.122 \\
\hline $\mathrm{N}$ & -0.666 & 1.853 & 7.022 \\
\hline C & 0.164 & 1.387 & 8.016 \\
\hline $\mathrm{H}$ & 1.080 & 0.874 & 7.772 \\
\hline C & 3.829 & 0.142 & 4.683 \\
\hline $\mathrm{H}$ & 4.684 & 0.741 & 4.351 \\
\hline $\mathrm{H}$ & 3.820 & -0.744 & 4.038 \\
\hline $\mathrm{C}$ & 2.546 & 0.943 & 4.324 \\
\hline $\mathrm{O}$ & 1.605 & 1.035 & 5.192 \\
\hline $\mathrm{O}$ & 2.559 & 1.449 & 3.182 \\
\hline C & 0.518 & 7.171 & 6.696 \\
\hline $\mathrm{H}$ & -0.369 & 7.599 & 6.221 \\
\hline $\mathrm{H}$ & 1.367 & 7.794 & 6.391 \\
\hline $\mathrm{N}$ & 1.937 & 5.099 & 6.271 \\
\hline $\mathrm{H}$ & 2.818 & 5.415 & 6.687 \\
\hline $\mathrm{C}$ & 0.718 & 5.763 & 6.233 \\
\hline $\mathrm{C}$ & 1.765 & 3.886 & 5.700 \\
\hline $\mathrm{H}$ & 2.540 & 3.143 & 5.640 \\
\hline $\mathrm{N}$ & 0.522 & 3.725 & 5.281 \\
\hline $\mathrm{C}$ & -0.139 & 4.888 & 5.612 \\
\hline $\mathrm{H}$ & -1.178 & 5.026 & 5.360 \\
\hline $\mathrm{C}$ & -4.148 & -2.931 & 3.957 \\
\hline $\mathrm{O}$ & -5.076 & -2.345 & 4.499 \\
\hline $\mathrm{N}$ & -2.837 & -2.605 & 3.771 \\
\hline $\mathrm{C}$ & -2.070 & -1.393 & 3.626 \\
\hline $\mathrm{H}$ & -2.716 & -0.520 & 3.727 \\
\hline $\mathrm{C}$ & -0.994 & -1.399 & 4.728 \\
\hline $\mathrm{O}$ & -0.432 & -2.495 & 4.943 \\
\hline $\mathrm{O}$ & -0.818 & -0.322 & 5.371 \\
\hline $\mathrm{C}$ & -1.448 & -1.428 & 2.163 \\
\hline $\mathrm{C}$ & -1.635 & -0.074 & 1.559 \\
\hline $\mathrm{H}$ & -1.007 & 0.713 & 2.446 \\
\hline $\mathrm{H}$ & -2.650 & 0.302 & 1.533 \\
\hline $\mathrm{H}$ & -1.067 & 0.108 & 0.647 \\
\hline $\mathrm{C}$ & 0.035 & -1.808 & 2.063 \\
\hline $\mathrm{H}$ & 0.239 & -2.796 & 2.478 \\
\hline $\mathrm{H}$ & 0.330 & -1.816 & 1.012 \\
\hline
\end{tabular}




\begin{tabular}{|c|c|c|c|}
\hline $\mathrm{H}$ & 0.628 & -1.033 & 2.564 \\
\hline S & -2.443 & -2.787 & 1.242 \\
\hline C & -2.544 & -3.686 & 2.854 \\
\hline $\mathrm{H}$ & -1.630 & -4.242 & 3.061 \\
\hline $\mathrm{C}$ & -3.893 & -4.313 & 3.299 \\
\hline $\mathrm{H}$ & -3.682 & -5.040 & 4.084 \\
\hline $\mathrm{N}$ & -4.726 & -4.956 & 2.334 \\
\hline $\mathrm{H}$ & -5.236 & -4.402 & 1.661 \\
\hline C & -4.309 & -6.224 & 1.977 \\
\hline $\mathrm{O}$ & -3.517 & -6.829 & 2.704 \\
\hline C & -4.762 & -6.788 & 0.671 \\
\hline $\mathrm{H}$ & -5.732 & -6.402 & 0.353 \\
\hline $\mathrm{H}$ & -4.855 & -7.865 & 0.826 \\
\hline C & -3.714 & -6.499 & -0.432 \\
\hline $\mathrm{H}$ & -3.915 & -5.509 & -0.857 \\
\hline $\mathrm{H}$ & -2.711 & -6.466 & 0.008 \\
\hline $\mathrm{C}$ & -3.740 & -7.576 & -1.520 \\
\hline $\mathrm{H}$ & -3.175 & -7.229 & -2.396 \\
\hline $\mathrm{H}$ & -3.217 & -8.463 & -1.160 \\
\hline $\mathrm{N}$ & -5.899 & -6.878 & -2.500 \\
\hline $\mathrm{H}$ & -5.441 & -6.623 & -3.387 \\
\hline $\mathrm{H}$ & -6.761 & -7.307 & -2.838 \\
\hline $\mathrm{C}$ & -5.137 & -8.009 & -1.962 \\
\hline $\mathrm{H}$ & -5.686 & -8.389 & -1.088 \\
\hline $\mathrm{C}$ & -5.056 & -9.186 & -2.958 \\
\hline $\mathrm{O}$ & -4.078 & -9.973 & -2.822 \\
\hline $\mathrm{O}$ & -5.940 & -9.251 & -3.843 \\
\hline $\mathrm{Fe}$ & -0.397 & 1.610 & 4.926 \\
\hline $\mathrm{O}$ & -0.229 & 1.357 & 3.142 \\
\hline $\mathrm{C}$ & -3.146 & 2.543 & 4.037 \\
\hline $\mathrm{O}$ & -3.395 & 1.637 & 3.214 \\
\hline $\mathrm{O}$ & -2.112 & 2.608 & 4.800 \\
\hline $\mathrm{C}$ & -4.155 & 3.671 & 4.205 \\
\hline $\mathrm{H}$ & -5.114 & 3.199 & 4.441 \\
\hline $\mathrm{H}$ & -3.863 & 4.300 & 5.050 \\
\hline $\mathrm{C}$ & -4.357 & 4.521 & 2.938 \\
\hline $\mathrm{H}$ & -3.952 & 3.998 & 2.070 \\
\hline $\mathrm{H}$ & -3.804 & 5.460 & 2.987 \\
\hline $\mathrm{C}$ & -5.814 & 4.832 & 2.573 \\
\hline $\mathrm{O}$ & -6.734 & 4.019 & 2.883 \\
\hline U & -5.996 & 5.904 & 1.925 \\
\hline
\end{tabular}

Coordinate of ${ }^{3} \mathrm{IM} 1 \mathrm{a}$

$\begin{array}{llll}\text { C } & 0.212 & 1.440 & 10.754\end{array}$

$\begin{array}{llll}\mathrm{H} & 0.883 & 0.579 & 10.773\end{array}$ 


\begin{tabular}{|c|c|c|c|}
\hline $\mathrm{H}$ & -0.581 & 1.253 & 484 \\
\hline N & -1.551 & 2.250 & 9.109 \\
\hline $\mathrm{H}$ & -2.176 & 2.652 & 9.811 \\
\hline & -0.342 & 1.626 & 9.376 \\
\hline${ }^{2}$ & -1.708 & 2.309 & 7.767 \\
\hline $\mathrm{H}$ & -2.560 & 2.737 & 7.265 \\
\hline N & -0.671 & 1.750 & 7.156 \\
\hline $\mathrm{C}$ & 0.188 & 1.327 & 8.145 \\
\hline $\mathrm{H}$ & 1.120 & 0.848 & 7.896 \\
\hline C & 3.728 & 0.039 & 4.735 \\
\hline $\mathrm{H}$ & 4.571 & 0.613 & 4.336 \\
\hline $\mathrm{H}$ & 3.679 & -0.875 & 4.134 \\
\hline $\mathrm{C}$ & 2.441 & 0.845 & 4.391 \\
\hline $\mathrm{O}$ & 1.510 & 0.940 & 5.276 \\
\hline $\mathrm{O}$ & 2.448 & 1.343 & 3.250 \\
\hline $\mathrm{C}$ & 0.523 & 7.157 & 6.696 \\
\hline $\mathrm{H}$ & -0.361 & 7.585 & 6.215 \\
\hline $\mathrm{H}$ & 1.378 & 7.768 & 6.384 \\
\hline $\mathrm{N}$ & 1.921 & 5.059 & 6.321 \\
\hline $\mathrm{H}$ & 2.809 & 5.387 & 6.711 \\
\hline $\mathrm{C}$ & 0.709 & 5.737 & 6.264 \\
\hline $\mathrm{C}$ & 1.725 & 3.820 & 5.819 \\
\hline $\mathrm{H}$ & 2.486 & 3.060 & 5.789 \\
\hline $\mathrm{N}$ & 0.475 & 3.658 & 5.423 \\
\hline $\mathrm{C}$ & -0.167 & 4.845 & 5.696 \\
\hline $\mathrm{H}$ & -1.207 & 4.982 & 5.446 \\
\hline $\mathrm{C}$ & -4.439 & -2.865 & 4.089 \\
\hline $\mathrm{O}$ & -5.387 & -2.205 & 4.505 \\
\hline $\mathrm{N}$ & -3.133 & -2.555 & 3.883 \\
\hline $\mathrm{C}$ & -2.332 & -1.399 & 3.539 \\
\hline $\mathrm{H}$ & -2.922 & -0.489 & 3.636 \\
\hline $\mathrm{C}$ & -1.180 & -1.369 & 4.580 \\
\hline $\mathrm{O}$ & -0.500 & -2.412 & 4.687 \\
\hline $\mathrm{O}$ & -1.122 & -0.347 & 5.333 \\
\hline $\mathrm{C}$ & -1.914 & -1.592 & 2.015 \\
\hline $\mathrm{C}$ & -2.727 & -0.727 & 1.154 \\
\hline $\mathrm{H}$ & -1.427 & 1.313 & 3.134 \\
\hline $\mathrm{H}$ & -3.760 & -0.503 & 1.396 \\
\hline $\mathrm{H}$ & -2.355 & -0.434 & 0.180 \\
\hline $\mathrm{C}$ & -0.417 & -1.461 & 1.719 \\
\hline $\mathrm{H}$ & 0.113 & -2.322 & 2.126 \\
\hline $\mathrm{H}$ & -0.256 & -1.456 & 0.637 \\
\hline $\mathrm{H}$ & -0.032 & -0.524 & 2.137 \\
\hline $\mathrm{S}$ & -2.439 & -3.424 & 1.553 \\
\hline$C$ & -2.757 & -3.834 & 3.306 \\
\hline
\end{tabular}




\begin{tabular}{|c|c|c|c|}
\hline $\mathrm{H}$ & -1.896 & -4.305 & 3.773 \\
\hline $\mathrm{C}$ & -4.173 & -4.346 & 3.705 \\
\hline $\mathrm{H}$ & -4.112 & -4.968 & 4.600 \\
\hline $\mathrm{N}$ & -4.926 & -5.025 & 2.685 \\
\hline $\mathrm{H}$ & -5.532 & -4.495 & 2.073 \\
\hline $\mathrm{C}$ & -4.340 & -6.184 & 2.226 \\
\hline $\mathrm{O}$ & -3.433 & -6.690 & 2.896 \\
\hline$C$ & -4.778 & -6.775 & 0.919 \\
\hline $\mathrm{H}$ & -5.736 & -6.379 & 0.577 \\
\hline $\mathrm{H}$ & -4.902 & -7.846 & 1.104 \\
\hline $\mathrm{C}$ & -3.710 & -6.557 & -0.182 \\
\hline $\mathrm{H}$ & -3.879 & -5.578 & -0.641 \\
\hline $\mathrm{H}$ & -2.715 & -6.537 & 0.276 \\
\hline $\mathrm{C}$ & -3.736 & -7.659 & -1.249 \\
\hline $\mathrm{H}$ & -3.091 & -7.364 & -2.087 \\
\hline $\mathrm{H}$ & -3.286 & -8.565 & -0.837 \\
\hline $\mathrm{N}$ & -5.810 & -6.828 & -2.304 \\
\hline $\mathrm{H}$ & -5.324 & -6.575 & -3.175 \\
\hline $\mathrm{H}$ & -6.685 & -7.206 & -2.669 \\
\hline $\mathrm{C}$ & -5.122 & -8.018 & -1.785 \\
\hline $\mathrm{H}$ & -5.731 & -8.411 & -0.957 \\
\hline $\mathrm{C}$ & -5.053 & -9.139 & -2.854 \\
\hline $\mathrm{O}$ & -4.072 & -9.932 & -2.799 \\
\hline $\mathrm{O}$ & -5.959 & -9.153 & -3.720 \\
\hline $\mathrm{Fe}$ & -0.449 & 1.581 & 5.094 \\
\hline $\mathrm{O}$ & -0.459 & 1.386 & 3.305 \\
\hline $\mathrm{C}$ & -3.123 & 2.559 & 4.109 \\
\hline $\mathrm{O}$ & -3.199 & 1.701 & 3.186 \\
\hline $\mathrm{O}$ & -2.208 & 2.590 & 5.003 \\
\hline $\mathrm{C}$ & -4.211 & 3.616 & 4.209 \\
\hline $\mathrm{H}$ & -5.147 & 3.088 & 4.423 \\
\hline $\mathrm{H}$ & -3.990 & 4.270 & 5.055 \\
\hline $\mathrm{C}$ & -4.415 & 4.423 & 2.919 \\
\hline $\mathrm{H}$ & -4.103 & 3.815 & 2.069 \\
\hline $\mathrm{H}$ & -3.785 & 5.314 & 2.898 \\
\hline $\mathrm{C}$ & -5.861 & 4.821 & 2.602 \\
\hline $\mathrm{O}$ & -6.811 & 4.044 & 2.916 \\
\hline $\mathrm{O}$ & -6.007 & 5.911 & 1.975 \\
\hline
\end{tabular}

Coordinate of ${ }^{3} \mathrm{TS} 2 \mathrm{a}$

$\begin{array}{llll}\text { C } & 0.220 & 1.418 & 10.843\end{array}$

$\begin{array}{llll}\mathrm{H} & 0.895 & 0.561 & 10.871\end{array}$

$\begin{array}{llll}\mathrm{H} & -0.561 & 1.243 & 11.589\end{array}$

$\begin{array}{llll}\mathrm{N} & -1.573 & 2.178 & 9.210\end{array}$

$\mathrm{H} \quad-2.196 \quad 2.582 \quad 9.913$ 


\begin{tabular}{|c|c|c|c|}
\hline C & -0.354 & 1.575 & 9.470 \\
\hline 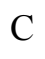 & -1.734 & 2.240 & 7.868 \\
\hline H & -2.594 & 2.657 & 7.372 \\
\hline & -0.691 & 1.700 & 7.251 \\
\hline & 0.176 & 1.287 & 8.236 \\
\hline$H$ & 1.113 & 0.817 & 7.986 \\
\hline & 3.705 & -0.088 & 4.816 \\
\hline & 4.506 & 0.548 & 4.422 \\
\hline$H$ & 3.743 & -1.006 & 4.221 \\
\hline $\mathrm{C}$ & 2.367 & 0.594 & 4.407 \\
\hline & 1.477 & 0.845 & 5.306 \\
\hline & 2.316 & 0.848 & 3.189 \\
\hline 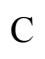 & 0.475 & 6.969 & 6.623 \\
\hline H & -0.421 & 7.364 & 6.138 \\
\hline 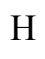 & 1.314 & 7.574 & 6.264 \\
\hline & 1.888 & 4.869 & 6.294 \\
\hline $\mathrm{H}$ & 2.782 & 5.230 & 6.642 \\
\hline $\mathcal{C}$ & 0.667 & 5.531 & 6.250 \\
\hline $\mathcal{C}$ & 1.701 & 3.611 & 5.849 \\
\hline & 2.463 & 2.853 & 5.824 \\
\hline $\mathbf{N}$ & 0.437 & 3.421 & 5.503 \\
\hline$C$ & -0.217 & 4.613 & 5.744 \\
\hline$H$ & -1.264 & 4.723 & 5.520 \\
\hline $\mathrm{C}$ & -4.323 & -2.845 & 4.065 \\
\hline$O$ & -5.256 & -2.205 & 4.548 \\
\hline$N$ & -3.045 & -2.508 & 3.768 \\
\hline & -2.279 & -1.309 & 3.501 \\
\hline $\mathrm{H}$ & -2.913 & -0.435 & 3.639 \\
\hline C & -1.143 & -1.266 & 4.558 \\
\hline U & -0.476 & -2.310 & 4.700 \\
\hline C & -1.093 & -0.232 & 5.301 \\
\hline C & -1.814 & -1.354 & 2.007 \\
\hline C & -2.630 & -0.590 & 1.138 \\
\hline & -1.397 & 1.445 & 3.204 \\
\hline $\mathrm{H}$ & -3.637 & -0.295 & 1.408 \\
\hline $\mathrm{H}$ & -2.305 & -0.400 & 0.123 \\
\hline C & -0.326 & -1.380 & 1.711 \\
\hline F & 0.121 & -2.303 & 2.077 \\
\hline $\mathrm{H}$ & -0.162 & -1.338 & 0.632 \\
\hline $\mathrm{H}$ & 0.152 & -0.505 & 2.172 \\
\hline & -2.454 & -3.439 & 1.391 \\
\hline $\mathrm{C}$ & -2.673 & -3.775 & 3.160 \\
\hline $\mathrm{H}$ & -1.781 & -4.226 & 3.592 \\
\hline & -4.056 & -4.314 & 3.649 \\
\hline & -3.925 & -4.944 & 4.531 \\
\hline
\end{tabular}




$\begin{array}{cccc}\mathrm{N} & -4.863 & -5.005 & 2.680 \\ \mathrm{H} & -5.436 & -4.464 & 2.049 \\ \mathrm{C} & -4.305 & -6.182 & 2.224 \\ \mathrm{O} & -3.405 & -6.707 & 2.888 \\ \mathrm{C} & -4.769 & -6.771 & 0.924 \\ \mathrm{H} & -5.730 & -6.370 & 0.598 \\ \mathrm{H} & -4.896 & -7.842 & 1.110 \\ \mathrm{C} & -3.714 & -6.558 & -0.192 \\ \mathrm{H} & -3.892 & -5.582 & -0.656 \\ \mathrm{H} & -2.715 & -6.528 & 0.255 \\ \mathrm{C} & -3.744 & -7.667 & -1.249 \\ \mathrm{H} & -3.095 & -7.381 & -2.088 \\ \mathrm{H} & -3.300 & -8.572 & -0.830 \\ \mathrm{~N} & -5.812 & -6.832 & -2.315 \\ \mathrm{H} & -5.321 & -6.582 & -3.184 \\ \mathrm{H} & -6.686 & -7.208 & -2.685 \\ \mathrm{C} & -5.129 & -8.022 & -1.789 \\ \mathrm{H} & -5.744 & -8.410 & -0.962 \\ \mathrm{C} & -5.056 & -9.144 & -2.856 \\ \mathrm{O} & -4.072 & -9.934 & -2.799 \\ \mathrm{O} & -5.959 & -9.160 & -3.725 \\ \mathrm{Fe} & -0.425 & 1.603 & 5.183 \\ \mathrm{O} & -0.442 & 1.598 & 3.370 \\ \mathrm{C} & -3.112 & 2.532 & 4.222 \\ \mathrm{O} & -3.173 & 1.717 & 3.259 \\ \mathrm{O} & -2.217 & 2.529 & 5.137 \\ \mathrm{C} & -4.195 & 3.596 & 4.324 \\ \mathrm{H} & -5.142 & 3.071 & 4.495 \\ \mathrm{H} & -3.996 & 4.222 & 5.198 \\ \mathrm{C} & -4.357 & 4.443 & 3.051 \\ \mathrm{H} & -3.945 & 3.897 & 2.201 \\ \mathrm{H} & -3.779 & 5.367 & 3.103 \\ \mathrm{C} & -5.799 & 4.784 & 2.644 \\ \mathrm{O} & -6.743 & 3.993 & 2.940 \\ \mathrm{O} & -5.943 & 5.848 & 1.973\end{array}$

Coordinate of ${ }^{3} \mathrm{IM} 2 \mathrm{a}$

$\begin{array}{llll}\text { C } & 0.213 & 1.439 & 10.812\end{array}$

$\begin{array}{llll}\mathrm{H} & 0.886 & 0.579 & 10.829\end{array}$

$\begin{array}{llll}\mathrm{H} & -0.574 & 1.253 & 11.548\end{array}$

$\begin{array}{llll}\mathrm{N} & -1.559 & 2.248 & 9.179\end{array}$

$\begin{array}{llll}\mathrm{H} & -2.183 & 2.646 & 9.885\end{array}$

$\begin{array}{llll}\text { C } & -0.349 & 1.621 & 9.438\end{array}$

$\begin{array}{llll}\text { C } & -1.719 & 2.322 & 7.840\end{array}$

$\begin{array}{llll}\mathrm{H} & -2.570 & 2.755 & 7.341\end{array}$ 


\begin{tabular}{|c|c|c|c|}
\hline $\mathrm{N}$ & -0.681 & 1.769 & 7.223 \\
\hline $\mathrm{C}$ & 0.179 & 1.333 & 8.204 \\
\hline $\mathrm{H}$ & 1.111 & 0.855 & 7.948 \\
\hline & 3.774 & -0.077 & 4.815 \\
\hline $\mathrm{H}$ & 4.588 & 0.548 & 4.430 \\
\hline $\mathrm{H}$ & 3.802 & -0.994 & 4.217 \\
\hline$C$ & 2.446 & 0.627 & 4.413 \\
\hline $\mathrm{O}$ & 1.579 & 0.928 & 5.311 \\
\hline $\mathrm{O}$ & 2.373 & 0.849 & 3.186 \\
\hline $\mathrm{C}$ & 0.524 & 7.135 & 6.698 \\
\hline $\mathrm{H}$ & -0.356 & 7.566 & 6.212 \\
\hline H & 1.383 & 7.741 & 6.387 \\
\hline $\mathrm{N}$ & 1.910 & 5.027 & 6.326 \\
\hline $\mathrm{H}$ & 2.807 & 5.360 & 6.691 \\
\hline C & 0.702 & 5.712 & 6.275 \\
\hline C & 1.705 & 3.783 & 5.845 \\
\hline $\mathrm{H}$ & 2.455 & 3.013 & 5.805 \\
\hline $\mathrm{N}$ & 0.446 & 3.623 & 5.472 \\
\hline $\mathrm{C}$ & -0.188 & 4.819 & 5.733 \\
\hline $\mathrm{H}$ & -1.229 & 4.958 & 5.492 \\
\hline $\mathrm{C}$ & -4.300 & -2.764 & 4.061 \\
\hline $\mathrm{O}$ & -5.216 & -2.134 & 4.588 \\
\hline $\mathrm{N}$ & -3.048 & -2.407 & 3.693 \\
\hline $\mathrm{C}$ & -2.262 & -1.197 & 3.531 \\
\hline $\mathrm{H}$ & -2.901 & -0.335 & 3.721 \\
\hline $\mathrm{C}$ & -1.154 & -1.245 & 4.613 \\
\hline $\mathrm{O}$ & -0.489 & -2.296 & 4.699 \\
\hline $\mathrm{O}$ & -1.101 & -0.257 & 5.416 \\
\hline $\mathrm{C}$ & -1.770 & -1.130 & 2.067 \\
\hline $\mathrm{C}$ & -2.642 & -0.616 & 1.156 \\
\hline $\mathrm{H}$ & -1.465 & 1.481 & 3.200 \\
\hline $\mathrm{H}$ & -3.650 & -0.326 & 1.428 \\
\hline $\mathrm{H}$ & -2.336 & -0.458 & 0.129 \\
\hline $\mathrm{C}$ & -0.323 & -1.373 & 1.724 \\
\hline $\mathrm{H}$ & 0.088 & -2.316 & 2.084 \\
\hline $\mathrm{H}$ & -0.166 & -1.328 & 0.643 \\
\hline $\mathrm{H}$ & 0.200 & -0.528 & 2.188 \\
\hline $\mathrm{S}$ & -2.493 & -3.437 & 1.327 \\
\hline $\mathrm{C}$ & -2.665 & -3.684 & 3.109 \\
\hline $\mathrm{H}$ & -1.761 & -4.107 & 3.545 \\
\hline $\mathrm{C}$ & -4.029 & -4.234 & 3.652 \\
\hline $\mathrm{H}$ & -3.857 & -4.847 & 4.539 \\
\hline $\mathrm{N}$ & -4.853 & -4.955 & 2.719 \\
\hline $\mathrm{H}$ & -5.436 & -4.427 & 2.084 \\
\hline C & -4.281 & -6.124 & 2.257 \\
\hline
\end{tabular}




$\begin{array}{cccc}\mathrm{O} & -3.359 & -6.627 & 2.909 \\ \mathrm{C} & -4.758 & -6.738 & 0.972 \\ \mathrm{H} & -5.716 & -6.332 & 0.641 \\ \mathrm{H} & -4.900 & -7.803 & 1.183 \\ \mathrm{C} & -3.705 & -6.566 & -0.152 \\ \mathrm{H} & -3.870 & -5.600 & -0.638 \\ \mathrm{H} & -2.704 & -6.541 & 0.294 \\ \mathrm{C} & -3.745 & -7.695 & -1.188 \\ \mathrm{H} & -3.074 & -7.440 & -2.019 \\ \mathrm{H} & -3.329 & -8.602 & -0.744 \\ \mathrm{~N} & -5.790 & -6.822 & -2.270 \\ \mathrm{H} & -5.292 & -6.574 & -3.136 \\ \mathrm{H} & -6.667 & -7.184 & -2.647 \\ \mathrm{C} & -5.128 & -8.027 & -1.750 \\ \mathrm{H} & -5.758 & -8.416 & -0.934 \\ \mathrm{C} & -5.058 & -9.136 & -2.833 \\ \mathrm{O} & -4.072 & -9.924 & -2.794 \\ \mathrm{O} & -5.964 & -9.140 & -3.698 \\ \mathrm{Fe} & -0.471 & 1.624 & 5.183 \\ \mathrm{O} & -0.495 & 1.506 & 3.383 \\ \mathrm{C} & -3.147 & 2.651 & 4.176 \\ \mathrm{O} & -3.196 & 1.819 & 3.223 \\ \mathrm{O} & -2.267 & 2.644 & 5.100 \\ \mathrm{C} & -4.227 & 3.710 & 4.262 \\ \mathrm{H} & -5.169 & 3.185 & 4.460 \\ \mathrm{H} & -4.014 & 4.356 & 5.117 \\ \mathrm{C} & -4.411 & 4.525 & 2.975 \\ \mathrm{H} & -4.022 & 3.958 & 2.128 \\ \mathrm{H} & -3.831 & 5.448 & 2.996 \\ \mathrm{C} & -5.861 & 4.853 & 2.607 \\ \mathrm{O} & -6.789 & 4.048 & 2.916 \\ \mathrm{O} & -6.032 & 5.925 & 1.956\end{array}$

Coordinate of ${ }^{3} \mathrm{TS} 3 \mathrm{a}$

$\begin{array}{llll}\text { C } & 0.230 & 1.429 & 10.842\end{array}$

$\begin{array}{llll}\mathrm{H} & 0.905 & 0.572 & 10.862\end{array}$

$\begin{array}{llll}\mathrm{H} & -0.550 & 1.249 & 11.588\end{array}$

$\begin{array}{llll}\mathrm{N} & -1.554 & 2.224 & 9.216\end{array}$

$\begin{array}{llll}\mathrm{H} & -2.176 & 2.623 & 9.922\end{array}$

$\begin{array}{llll}\text { C } & -0.346 & 1.597 & 9.471\end{array}$

$\begin{array}{llll}\text { C } & -1.714 & 2.300 & 7.875\end{array}$

$\begin{array}{llll}\mathrm{H} & -2.566 & 2.737 & 7.384\end{array}$

$\begin{array}{llll}\mathrm{N} & -0.681 & 1.746 & 7.254\end{array}$

$\begin{array}{llll}\text { C } & 0.178 & 1.307 & 8.235\end{array}$

$\begin{array}{llll}\mathrm{H} & 1.106 & 0.822 & 7.980\end{array}$ 


\begin{tabular}{|c|c|c|c|}
\hline $\mathrm{C}$ & 3.674 & -0.108 & 4.813 \\
\hline $\mathrm{H}$ & 4.503 & 0.460 & 4.377 \\
\hline $\mathrm{H}$ & 3.631 & -1.047 & 4.251 \\
\hline $\mathrm{C}$ & 2.378 & 0.656 & 4.418 \\
\hline $\mathrm{O}$ & 1.480 & 0.893 & 5.312 \\
\hline O & 2.358 & 0.969 & 3.212 \\
\hline C & 0.499 & 7.023 & 6.642 \\
\hline $\mathrm{H}$ & -0.391 & 7.428 & 6.153 \\
\hline $\mathrm{H}$ & 1.346 & 7.628 & 6.300 \\
\hline $\mathrm{N}$ & 1.907 & 4.923 & 6.302 \\
\hline $\mathrm{H}$ & 2.803 & 5.280 & 6.648 \\
\hline C & 0.689 & 5.589 & 6.256 \\
\hline $\mathrm{C}$ & 1.717 & 3.664 & 5.860 \\
\hline $\mathrm{H}$ & 2.478 & 2.907 & 5.833 \\
\hline $\mathrm{N}$ & 0.451 & 3.478 & 5.516 \\
\hline $\mathrm{C}$ & -0.198 & 4.672 & 5.755 \\
\hline $\mathrm{H}$ & -1.244 & 4.786 & 5.530 \\
\hline $\mathrm{C}$ & -4.059 & -2.747 & 3.796 \\
\hline $\mathrm{O}$ & -4.889 & -2.214 & 4.529 \\
\hline $\mathrm{N}$ & -2.923 & -2.275 & 3.222 \\
\hline $\mathrm{C}$ & -2.130 & -1.056 & 3.309 \\
\hline $\mathrm{H}$ & -2.799 & -0.213 & 3.480 \\
\hline $\mathrm{C}$ & -1.162 & -1.169 & 4.512 \\
\hline $\mathrm{O}$ & -0.623 & -2.279 & 4.694 \\
\hline $\mathrm{O}$ & -1.068 & -0.162 & 5.287 \\
\hline $\mathrm{C}$ & -1.465 & -0.890 & 1.944 \\
\hline $\mathrm{C}$ & -2.293 & -0.720 & 0.886 \\
\hline $\mathrm{H}$ & -1.376 & 1.575 & 3.205 \\
\hline $\mathrm{H}$ & -3.344 & -0.492 & 1.014 \\
\hline $\mathrm{H}$ & -1.895 & -0.657 & -0.118 \\
\hline $\mathrm{C}$ & -0.004 & -1.151 & 1.758 \\
\hline $\mathrm{H}$ & 0.208 & -2.203 & 1.988 \\
\hline $\mathrm{H}$ & 0.284 & -0.991 & 0.718 \\
\hline $\mathrm{H}$ & 0.595 & -0.486 & 2.392 \\
\hline $\mathrm{S}$ & -2.842 & -3.286 & 0.664 \\
\hline $\mathrm{C}$ & -2.588 & -3.479 & 2.475 \\
\hline $\mathrm{H}$ & -1.591 & -3.861 & 2.700 \\
\hline $\mathrm{C}$ & -3.773 & -4.161 & 3.245 \\
\hline $\mathrm{H}$ & -3.372 & -4.779 & 4.050 \\
\hline $\mathrm{N}$ & -4.695 & -4.960 & 2.489 \\
\hline $\mathrm{H}$ & -5.230 & -4.483 & 1.775 \\
\hline $\mathrm{C}$ & -4.212 & -6.216 & 2.158 \\
\hline $\mathrm{O}$ & -3.350 & -6.739 & 2.869 \\
\hline $\mathrm{C}$ & -4.694 & -6.864 & 0.897 \\
\hline $\mathrm{H}$ & -5.685 & -6.515 & 0.601 \\
\hline
\end{tabular}




$\begin{array}{lccc}\mathrm{H} & -4.748 & -7.937 & 1.097 \\ \mathrm{C} & -3.694 & -6.586 & -0.255 \\ \mathrm{H} & -3.930 & -5.610 & -0.692 \\ \mathrm{H} & -2.677 & -6.528 & 0.148 \\ \mathrm{C} & -3.730 & -7.668 & -1.334 \\ \mathrm{H} & -3.121 & -7.345 & -2.188 \\ \mathrm{H} & -3.252 & -8.572 & -0.953 \\ \mathrm{~N} & -5.832 & -6.858 & -2.342 \\ \mathrm{H} & -5.363 & -6.606 & -3.223 \\ \mathrm{H} & -6.714 & -7.238 & -2.686 \\ \mathrm{C} & -5.126 & -8.039 & -1.830 \\ \mathrm{H} & -5.712 & -8.431 & -0.985 \\ \mathrm{C} & -5.058 & -9.167 & -2.887 \\ \mathrm{O} & -4.076 & -9.959 & -2.814 \\ \mathrm{O} & -5.954 & -9.187 & -3.763 \\ \mathrm{Fe} & -0.413 & 1.673 & 5.190 \\ \mathrm{O} & -0.420 & 1.699 & 3.378 \\ \mathrm{C} & -3.092 & 2.619 & 4.238 \\ \mathrm{O} & -3.160 & 1.791 & 3.287 \\ \mathrm{O} & -2.201 & 2.613 & 5.153 \\ \mathrm{C} & -4.162 & 3.696 & 4.314 \\ \mathrm{H} & -5.105 & 3.187 & 4.546 \\ \mathrm{H} & -3.932 & 4.372 & 5.141 \\ \mathrm{C} & -4.358 & 4.461 & 2.995 \\ \mathrm{H} & -4.015 & 3.833 & 2.171 \\ \mathrm{H} & -3.745 & 5.362 & 2.955 \\ \mathrm{C} & -5.806 & 4.818 & 2.637 \\ \mathrm{O} & -6.745 & 4.032 & 2.961 \\ \mathrm{O} & -5.962 & 5.883 & 1.970\end{array}$

Coordinate of ${ }^{3} \mathrm{IM} 3 \mathrm{a}$

$\begin{array}{cccc}\mathrm{C} & 0.227 & 1.431 & 10.843 \\ \mathrm{H} & 0.900 & 0.573 & 10.865 \\ \mathrm{H} & -0.556 & 1.252 & 11.587 \\ \mathrm{~N} & -1.552 & 2.232 & 9.214 \\ \mathrm{H} & -2.175 & 2.630 & 9.921 \\ \mathrm{C} & -0.345 & 1.602 & 9.471 \\ \mathrm{C} & -1.706 & 2.314 & 7.874 \\ \mathrm{H} & -2.557 & 2.752 & 7.381 \\ \mathrm{~N} & -0.672 & 1.762 & 7.253 \\ \mathrm{C} & 0.183 & 1.317 & 8.236 \\ \mathrm{H} & 1.111 & 0.832 & 7.982 \\ \mathrm{C} & 3.682 & -0.099 & 4.809 \\ \mathrm{H} & 4.514 & 0.467 & 4.376 \\ \mathrm{H} & 3.635 & -1.037 & 4.245\end{array}$




\begin{tabular}{|c|c|c|c|}
\hline$C_{1}$ & 2.388 & 0.671 & 4.414 \\
\hline О & 1.492 & 0.912 & 5.309 \\
\hline & 2.372 & 0.989 & 3.209 \\
\hline${ }^{2}$ & 0.500 & 7.037 & 6.647 \\
\hline $\mathrm{H}$ & -0.390 & 7.442 & 6.159 \\
\hline $\mathrm{H}$ & 1.347 & 7.644 & 6.307 \\
\hline $\mathrm{N}$ & 1.912 & 4.940 & 6.302 \\
\hline $\mathrm{H}$ & 2.807 & 5.295 & 6.651 \\
\hline $\mathrm{C}$ & 0.692 & 5.604 & 6.257 \\
\hline $\mathrm{C}$ & 1.723 & 3.682 & 5.857 \\
\hline $\mathrm{H}$ & 2.484 & 2.925 & 5.828 \\
\hline $\mathrm{N}$ & 0.458 & 3.496 & 5.511 \\
\hline $\mathrm{C}$ & -0.193 & 4.688 & 5.752 \\
\hline $\mathrm{H}$ & -1.240 & 4.800 & 5.528 \\
\hline $\mathrm{C}$ & -4.095 & -2.752 & 3.819 \\
\hline $\mathrm{O}$ & -4.948 & -2.232 & 4.534 \\
\hline $\mathrm{N}$ & -2.936 & -2.275 & 3.296 \\
\hline $\mathrm{C}$ & -2.144 & -1.048 & 3.328 \\
\hline $\mathrm{H}$ & -2.813 & -0.203 & 3.501 \\
\hline $\mathrm{C}$ & -1.153 & -1.147 & 4.513 \\
\hline $\mathrm{O}$ & -0.608 & -2.256 & 4.691 \\
\hline $\mathrm{O}$ & -1.053 & -0.141 & 5.288 \\
\hline $\mathrm{C}$ & -1.530 & -0.938 & 1.945 \\
\hline $\mathrm{C}$ & -2.485 & -1.220 & 0.852 \\
\hline $\mathrm{H}$ & -1.356 & 1.550 & 3.202 \\
\hline $\mathrm{H}$ & -3.450 & -0.730 & 0.999 \\
\hline $\mathrm{H}$ & -2.084 & -0.952 & -0.126 \\
\hline $\mathrm{C}$ & -0.071 & -1.129 & 1.699 \\
\hline $\mathrm{H}$ & 0.216 & -2.178 & 1.880 \\
\hline $\mathrm{H}$ & 0.168 & -0.935 & 0.651 \\
\hline $\mathrm{H}$ & 0.534 & -0.458 & 2.320 \\
\hline $\mathrm{S}$ & -2.835 & -3.098 & 0.706 \\
\hline $\mathrm{C}$ & -2.604 & -3.443 & 2.513 \\
\hline $\mathrm{H}$ & -1.606 & -3.838 & 2.708 \\
\hline $\mathrm{C}$ & -3.791 & -4.155 & 3.233 \\
\hline $\mathrm{H}$ & -3.399 & -4.790 & 4.029 \\
\hline $\mathrm{N}$ & -4.688 & -4.946 & 2.445 \\
\hline $\mathrm{H}$ & -5.225 & -4.471 & 1.730 \\
\hline $\mathrm{C}$ & -4.210 & -6.207 & 2.131 \\
\hline $\mathrm{O}$ & -3.358 & -6.731 & 2.853 \\
\hline $\mathrm{C}$ & -4.683 & -6.856 & 0.868 \\
\hline $\mathrm{H}$ & -5.675 & -6.513 & 0.568 \\
\hline $\mathrm{H}$ & -4.729 & -7.930 & 1.066 \\
\hline $\mathrm{C}$ & -3.681 & -6.568 & -0.280 \\
\hline H & -3.917 & -5.591 & -0.713 \\
\hline
\end{tabular}




$\begin{array}{lccc}\mathrm{H} & -2.664 & -6.512 & 0.125 \\ \mathrm{C} & -3.718 & -7.652 & -1.358 \\ \mathrm{H} & -3.116 & -7.330 & -2.218 \\ \mathrm{H} & -3.235 & -8.553 & -0.977 \\ \mathrm{~N} & -5.834 & -6.855 & -2.348 \\ \mathrm{H} & -5.374 & -6.604 & -3.234 \\ \mathrm{H} & -6.717 & -7.241 & -2.683 \\ \mathrm{C} & -5.117 & -8.031 & -1.840 \\ \mathrm{H} & -5.693 & -8.424 & -0.988 \\ \mathrm{C} & -5.055 & -9.164 & -2.891 \\ \mathrm{O} & -4.076 & -9.959 & -2.814 \\ \mathrm{O} & -5.953 & -9.186 & -3.764 \\ \mathrm{Fe} & -0.402 & 1.690 & 5.189 \\ \mathrm{O} & -0.407 & 1.714 & 3.379 \\ \mathrm{C} & -3.084 & 2.621 & 4.237 \\ \mathrm{O} & -3.153 & 1.783 & 3.295 \\ \mathrm{O} & -2.192 & 2.625 & 5.151 \\ \mathrm{C} & -4.156 & 3.697 & 4.303 \\ \mathrm{H} & -5.098 & 3.190 & 4.547 \\ \mathrm{H} & -3.924 & 4.385 & 5.119 \\ \mathrm{C} & -4.358 & 4.442 & 2.974 \\ \mathrm{H} & -4.032 & 3.795 & 2.157 \\ \mathrm{H} & -3.737 & 5.337 & 2.914 \\ \mathrm{C} & -5.806 & 4.811 & 2.629 \\ \mathrm{O} & -6.748 & 4.030 & 2.962 \\ \mathrm{O} & -5.963 & 5.878 & 1.967\end{array}$

Coordinate of ${ }^{3} \mathrm{TS} 4 \mathrm{a}$

$\begin{array}{lrrr}\mathrm{C} & 0.232 & 1.395 & 10.824 \\ \mathrm{H} & 0.913 & 0.543 & 10.845 \\ \mathrm{H} & -0.546 & 1.211 & 11.572 \\ \mathrm{~N} & -1.562 & 2.181 & 9.209 \\ \mathrm{H} & -2.174 & 2.592 & 9.919 \\ \mathrm{C} & -0.352 & 1.549 & 9.456 \\ \mathrm{C} & -1.754 & 2.227 & 7.876 \\ \mathrm{H} & -2.608 & 2.663 & 7.386 \\ \mathrm{~N} & -0.737 & 1.642 & 7.248 \\ \mathrm{C} & 0.145 & 1.222 & 8.219 \\ \mathrm{H} & 1.069 & 0.734 & 7.952 \\ \mathrm{C} & 3.843 & -0.056 & 4.787 \\ \mathrm{H} & 4.666 & 0.572 & 4.427 \\ \mathrm{H} & 3.874 & -0.961 & 4.172 \\ \mathrm{C} & 2.522 & 0.666 & 4.396 \\ \mathrm{O} & 1.630 & 0.904 & 5.277 \\ \mathrm{O} & 2.477 & 0.959 & 3.176\end{array}$




\begin{tabular}{|c|c|c|c|}
\hline & 0.467 & 7.060 & 6.667 \\
\hline H & -0.415 & 7.492 & 6.187 \\
\hline H & 1.328 & 7.649 & 6.332 \\
\hline & 1.825 & 4.931 & 6.319 \\
\hline H & 2.727 & 5.270 & 6.666 \\
\hline $\mathrm{C}$ & 0.622 & 5.626 & 6.267 \\
\hline$C$ & 1.607 & 3.680 & 5.869 \\
\hline $\mathrm{H}$ & 2.346 & 2.899 & 5.839 \\
\hline $\mathrm{N}$ & 0.340 & 3.522 & 5.514 \\
\hline $\mathrm{C}$ & -0.282 & 4.731 & 5.755 \\
\hline $\mathrm{H}$ & -1.325 & 4.873 & 5.525 \\
\hline $\mathrm{C}$ & -3.718 & -2.418 & 3.647 \\
\hline $\mathrm{O}$ & -4.484 & -1.920 & 4.466 \\
\hline $\mathrm{N}$ & -2.586 & -1.975 & 3.010 \\
\hline $\mathrm{C}$ & -1.458 & -1.013 & 3.082 \\
\hline $\mathrm{H}$ & -1.575 & 0.213 & 3.176 \\
\hline $\mathrm{C}$ & -0.796 & -1.317 & 4.445 \\
\hline $\mathrm{O}$ & -0.375 & -2.465 & 4.675 \\
\hline $\mathrm{O}$ & -0.873 & -0.396 & 5.348 \\
\hline $\mathrm{C}$ & -0.745 & -1.171 & 1.842 \\
\hline $\mathrm{C}$ & -1.594 & -1.238 & 0.629 \\
\hline $\mathrm{H}$ & -1.664 & 1.808 & 3.268 \\
\hline $\mathrm{H}$ & -2.391 & -0.490 & 0.642 \\
\hline $\mathrm{H}$ & -1.017 & -1.151 & -0.292 \\
\hline $\mathrm{C}$ & 0.700 & -1.525 & 1.745 \\
\hline $\mathrm{H}$ & 0.863 & -2.593 & 1.972 \\
\hline $\mathrm{H}$ & 1.079 & -1.376 & 0.733 \\
\hline $\mathrm{H}$ & 1.307 & -0.891 & 2.405 \\
\hline $\mathrm{S}$ & -2.509 & -2.929 & 0.479 \\
\hline $\mathrm{C}$ & -2.340 & -3.217 & 2.284 \\
\hline $\mathrm{H}$ & -1.365 & -3.650 & 2.516 \\
\hline $\mathrm{C}$ & -3.525 & -3.836 & 3.053 \\
\hline $\mathrm{H}$ & -3.136 & -4.475 & 3.849 \\
\hline $\mathrm{N}$ & -4.483 & -4.622 & 2.324 \\
\hline $\mathrm{H}$ & -4.977 & -4.172 & 1.566 \\
\hline $\mathrm{C}$ & -4.117 & -5.944 & 2.132 \\
\hline $\mathrm{O}$ & -3.320 & -6.477 & 2.910 \\
\hline $\mathrm{C}$ & -4.632 & -6.665 & 0.921 \\
\hline $\mathrm{H}$ & -5.601 & -6.292 & 0.585 \\
\hline $\mathrm{H}$ & -4.742 & -7.715 & 1.204 \\
\hline $\mathrm{C}$ & -3.609 & -6.527 & -0.239 \\
\hline $\mathrm{H}$ & -3.784 & -5.573 & -0.746 \\
\hline $\mathrm{H}$ & -2.594 & -6.497 & 0.174 \\
\hline $\mathrm{C}$ & -3.686 & -7.682 & -1.242 \\
\hline $\mathrm{H}$ & -3.046 & -7.449 & -2.102 \\
\hline
\end{tabular}




$\begin{array}{llll}\mathrm{H} & -3.255 & -8.577 & -0.790 \\ \mathrm{~N} & -5.785 & -6.821 & -2.222 \\ \mathrm{H} & -5.341 & -6.578 & -3.118 \\ \mathrm{H} & -6.688 & -7.180 & -2.533 \\ \mathrm{C} & -5.091 & -8.023 & -1.740 \\ \mathrm{H} & -5.677 & -8.421 & -0.897 \\ \mathrm{C} & -5.052 & -9.130 & -2.826 \\ \mathrm{O} & -4.075 & -9.930 & -2.797 \\ \mathrm{O} & -5.968 & -9.122 & -3.681 \\ \mathrm{Fe} & -0.580 & 1.551 & 5.226 \\ \mathrm{O} & -0.755 & 1.431 & 3.406 \\ \mathrm{C} & -3.247 & 2.736 & 4.235 \\ \mathrm{O} & -3.255 & 1.977 & 3.221 \\ \mathrm{O} & -2.414 & 2.628 & 5.196 \\ \mathrm{C} & -4.292 & 3.821 & 4.305 \\ \mathrm{H} & -5.235 & 3.330 & 4.576 \\ \mathrm{H} & -4.031 & 4.514 & 5.108 \\ \mathrm{C} & -4.501 & 4.540 & 2.966 \\ \mathrm{H} & -4.171 & 3.877 & 2.163 \\ \mathrm{H} & -3.886 & 5.437 & 2.890 \\ \mathrm{C} & -5.948 & 4.885 & 2.628 \\ \mathrm{O} & -6.876 & 4.084 & 2.953 \\ \mathrm{O} & -6.122 & 5.957 & 1.979\end{array}$

Coordinate of ${ }^{3} \mathrm{~Pa}$

$\begin{array}{cccc}\mathrm{C} & 0.196 & 1.458 & 10.957 \\ \mathrm{H} & 0.860 & 0.593 & 10.978 \\ \mathrm{H} & -0.552 & 1.312 & 11.741 \\ \mathrm{~N} & -1.657 & 2.188 & 9.365 \\ \mathrm{H} & -2.261 & 2.615 & 10.075 \\ \mathrm{C} & -0.436 & 1.582 & 9.607 \\ \mathrm{C} & -1.858 & 2.213 & 8.026 \\ \mathrm{H} & -2.719 & 2.636 & 7.533 \\ \mathrm{~N} & -0.841 & 1.639 & 7.398 \\ \mathrm{C} & 0.052 & 1.248 & 8.370 \\ \mathrm{H} & 0.986 & 0.775 & 8.107 \\ \mathrm{C} & 3.781 & -0.164 & 4.768 \\ \mathrm{H} & 4.565 & 0.467 & 4.336 \\ \mathrm{H} & 3.779 & -1.085 & 4.173 \\ \mathrm{C} & 2.413 & 0.510 & 4.512 \\ \mathrm{O} & 1.610 & 0.707 & 5.465 \\ \mathrm{O} & 2.186 & 0.797 & 3.289 \\ \mathrm{C} & 0.577 & 7.088 & 6.721 \\ \mathrm{H} & -0.301 & 7.479 & 6.200 \\ \mathrm{H} & 1.436 & 7.683 & 6.388\end{array}$




\begin{tabular}{|c|c|c|c|}
\hline & 1.988 & 4.974 & 6.466 \\
\hline $\mathrm{H}$ & 2.889 & 5.337 & 6.790 \\
\hline $\mathrm{C}$ & 0.773 & 5.643 & 6.389 \\
\hline $\mathrm{C}$ & 1.778 & 3.689 & 6.106 \\
\hline $\mathrm{H}$ & 2.536 & 2.923 & 6.112 \\
\hline $\mathrm{N}$ & 0.512 & 3.494 & 5.777 \\
\hline $\mathrm{C}$ & -0.123 & 4.703 & 5.951 \\
\hline $\mathrm{H}$ & -1.172 & 4.808 & 5.724 \\
\hline $\mathrm{C}$ & -4.367 & -3.005 & 3.913 \\
\hline $\mathrm{O}$ & -5.129 & -2.427 & 4.678 \\
\hline $\mathrm{N}$ & -3.234 & -2.579 & 3.285 \\
\hline C & -2.258 & -1.554 & 3.284 \\
\hline $\mathrm{H}$ & 0.527 & 1.240 & 3.248 \\
\hline $\mathrm{C}$ & -1.512 & -1.425 & 4.602 \\
\hline $\mathrm{O}$ & -1.281 & -2.536 & 5.147 \\
\hline $\mathrm{O}$ & -1.187 & -0.288 & 5.024 \\
\hline $\mathrm{C}$ & -1.870 & -1.232 & 2.030 \\
\hline $\mathrm{C}$ & -2.785 & -1.626 & 0.897 \\
\hline $\mathrm{H}$ & -1.150 & 1.381 & 3.206 \\
\hline $\mathrm{H}$ & -3.758 & -1.142 & 1.008 \\
\hline $\mathrm{H}$ & -2.358 & -1.338 & -0.065 \\
\hline $\mathrm{C}$ & -0.545 & -0.664 & 1.632 \\
\hline $\mathrm{H}$ & -0.085 & -1.339 & 0.899 \\
\hline $\mathrm{H}$ & -0.666 & 0.308 & 1.140 \\
\hline $\mathrm{H}$ & 0.153 & -0.575 & 2.463 \\
\hline $\mathrm{S}$ & -3.095 & -3.464 & 0.753 \\
\hline $\mathrm{C}$ & -2.964 & -3.806 & 2.559 \\
\hline $\mathrm{H}$ & -1.984 & -4.227 & 2.793 \\
\hline $\mathrm{C}$ & -4.168 & -4.434 & 3.322 \\
\hline $\mathrm{H}$ & -3.795 & -5.098 & 4.104 \\
\hline $\mathrm{N}$ & -5.125 & -5.173 & 2.550 \\
\hline $\mathrm{H}$ & -5.566 & -4.659 & 1.796 \\
\hline $\mathrm{C}$ & -4.699 & -6.455 & 2.215 \\
\hline $\mathrm{O}$ & -3.956 & -7.075 & 2.973 \\
\hline $\mathrm{C}$ & -5.064 & -6.992 & 0.865 \\
\hline $\mathrm{H}$ & -6.020 & -6.611 & 0.501 \\
\hline $\mathrm{H}$ & -5.137 & -8.073 & 0.978 \\
\hline C & -3.963 & -6.655 & -0.173 \\
\hline $\mathrm{H}$ & -4.179 & -5.678 & -0.613 \\
\hline $\mathrm{H}$ & -2.988 & -6.589 & 0.325 \\
\hline $\mathrm{C}$ & -3.883 & -7.717 & -1.270 \\
\hline $\mathrm{H}$ & -3.195 & -7.379 & -2.057 \\
\hline $\mathrm{H}$ & -3.440 & -8.626 & -0.860 \\
\hline $\mathrm{N}$ & -5.878 & -6.902 & -2.484 \\
\hline $\mathrm{H}$ & -5.326 & -6.641 & -3.312 \\
\hline
\end{tabular}




$\begin{array}{lccc}\mathrm{H} & -6.712 & -7.290 & -2.927 \\ \mathrm{C} & -5.226 & -8.081 & -1.898 \\ \mathrm{H} & -5.895 & -8.464 & -1.113 \\ \mathrm{C} & -5.074 & -9.212 & -2.938 \\ \mathrm{O} & -4.069 & -9.967 & -2.822 \\ \mathrm{O} & -5.938 & -9.270 & -3.844 \\ \mathrm{Fe} & -0.508 & 1.649 & 5.431 \\ \mathrm{O} & -0.280 & 1.784 & 3.460 \\ \mathrm{C} & -3.059 & 2.619 & 4.104 \\ \mathrm{O} & -2.894 & 1.762 & 3.181 \\ \mathrm{O} & -2.344 & 2.716 & 5.147 \\ \mathrm{C} & -4.227 & 3.602 & 3.999 \\ \mathrm{H} & -5.056 & 3.179 & 4.584 \\ \mathrm{H} & -3.931 & 4.526 & 4.504 \\ \mathrm{C} & -4.697 & 3.868 & 2.568 \\ \mathrm{H} & -4.814 & 2.894 & 2.080 \\ \mathrm{H} & -3.940 & 4.436 & 2.020 \\ \mathrm{C} & -6.038 & 4.589 & 2.466 \\ \mathrm{O} & -7.078 & 3.957 & 2.835 \\ \mathrm{O} & -6.040 & 5.761 & 1.988\end{array}$

\begin{tabular}{lrrr}
\multicolumn{5}{c}{ Coordinate of ${ }^{3}$ TS4a-reb } \\
C & 0.203 & 1.449 & 10.848 \\
H & 0.865 & 0.581 & 10.857 \\
H & -0.577 & 1.277 & 11.595 \\
N & -1.557 & 2.313 & 9.222 \\
H & -2.178 & 2.713 & 9.931 \\
$\mathrm{C}$ & -0.369 & 1.646 & 9.478 \\
$\mathrm{C}$ & -1.693 & 2.426 & 7.880 \\
$\mathrm{H}$ & -2.533 & 2.887 & 7.388 \\
$\mathrm{~N}$ & -0.668 & 1.859 & 7.261 \\
$\mathrm{C}$ & 0.161 & 1.370 & 8.242 \\
$\mathrm{H}$ & 1.078 & 0.861 & 7.990 \\
$\mathrm{C}$ & 3.670 & -0.165 & 4.806 \\
$\mathrm{H}$ & 4.524 & 0.337 & 4.340 \\
$\mathrm{H}$ & 3.556 & -1.119 & 4.278 \\
$\mathrm{C}$ & 2.425 & 0.674 & 4.411 \\
$\mathrm{O}$ & 1.512 & 0.913 & 5.284 \\
$\mathrm{O}$ & 2.446 & 1.042 & 3.220 \\
$\mathrm{C}$ & 0.494 & 7.031 & 6.628 \\
$\mathrm{H}$ & -0.399 & 7.440 & 6.147 \\
$\mathrm{H}$ & 1.336 & 7.645 & 6.290 \\
$\mathrm{~N}$ & 1.914 & 4.948 & 6.242 \\
$\mathrm{H}$ & 2.806 & 5.298 & 6.605
\end{tabular}




\begin{tabular}{|c|c|c|c|}
\hline & 0.690 & 5.606 & 6.215 \\
\hline $\mathrm{C}$ & 1.732 & 3.703 & 5.757 \\
\hline $\mathrm{H}$ & 2.503 & 2.956 & 5.710 \\
\hline & 0.469 & 3.515 & 5.406 \\
\hline $\mathrm{C}$ & -0.188 & 4.697 & 5.685 \\
\hline $\mathrm{H}$ & -1.236 & 4.811 & 5.466 \\
\hline$C$ & -3.908 & -2.695 & 3.760 \\
\hline $\mathrm{O}$ & -4.779 & -2.243 & 4.499 \\
\hline $\mathrm{N}$ & -2.735 & -2.174 & 3.315 \\
\hline $\mathrm{C}$ & -1.966 & -0.956 & 3.502 \\
\hline $\mathrm{H}$ & -2.670 & -0.152 & 3.709 \\
\hline C & -1.039 & -1.140 & 4.705 \\
\hline $\mathrm{O}$ & -0.520 & -2.263 & 4.860 \\
\hline $\mathrm{O}$ & -0.947 & -0.158 & 5.512 \\
\hline C & -1.255 & -0.669 & 2.190 \\
\hline $\mathrm{C}$ & -2.194 & -0.766 & 1.041 \\
\hline $\mathrm{H}$ & -1.569 & 1.438 & 3.151 \\
\hline $\mathrm{H}$ & -3.144 & -0.262 & 1.213 \\
\hline $\mathrm{H}$ & -1.753 & -0.428 & 0.105 \\
\hline $\mathrm{C}$ & 0.138 & -1.155 & 1.920 \\
\hline $\mathrm{H}$ & 0.175 & -2.257 & 1.928 \\
\hline $\mathrm{H}$ & 0.451 & -0.865 & 0.916 \\
\hline $\mathrm{H}$ & 0.854 & -0.732 & 2.624 \\
\hline S & -2.636 & -2.623 & 0.656 \\
\hline $\mathrm{C}$ & -2.410 & -3.233 & 2.392 \\
\hline $\mathrm{H}$ & -1.415 & -3.661 & 2.528 \\
\hline $\mathrm{C}$ & -3.586 & -4.028 & 3.042 \\
\hline $\mathrm{H}$ & -3.167 & -4.714 & 3.783 \\
\hline $\mathrm{N}$ & -4.469 & -4.796 & 2.229 \\
\hline $\mathrm{H}$ & -4.945 & -4.326 & 1.470 \\
\hline $\mathrm{C}$ & -4.103 & -6.115 & 2.032 \\
\hline $\mathrm{O}$ & -3.344 & -6.669 & 2.831 \\
\hline $\mathrm{C}$ & -4.583 & -6.792 & 0.790 \\
\hline $\mathrm{H}$ & -5.578 & -6.455 & 0.491 \\
\hline $\mathrm{H}$ & -4.626 & -7.860 & 1.011 \\
\hline $\mathrm{C}$ & -3.595 & -6.518 & -0.372 \\
\hline $\mathrm{H}$ & -3.824 & -5.538 & -0.805 \\
\hline $\mathrm{H}$ & -2.571 & -6.473 & 0.016 \\
\hline $\mathrm{C}$ & -3.666 & -7.610 & -1.440 \\
\hline $\mathrm{H}$ & -3.106 & -7.288 & -2.329 \\
\hline $\mathrm{H}$ & -3.160 & -8.504 & -1.076 \\
\hline $\mathrm{N}$ & -5.832 & -6.841 & -2.335 \\
\hline $\mathrm{H}$ & -5.414 & -6.593 & -3.243 \\
\hline $\mathrm{H}$ & -6.730 & -7.233 & -2.622 \\
\hline$C$ & -5.082 & -8.007 & -1.8 \\
\hline
\end{tabular}




$\begin{array}{llll}\mathrm{H} & -5.612 & -8.402 & -0.977 \\ \mathrm{C} & -5.049 & -9.151 & -2.897 \\ \mathrm{O} & -4.081 & -9.959 & -2.815 \\ \mathrm{O} & -5.955 & -9.169 & -3.760 \\ \mathrm{Fe} & -0.395 & 1.663 & 5.172 \\ \mathrm{O} & -0.607 & 1.253 & 3.158 \\ \mathrm{C} & -3.105 & 2.663 & 4.198 \\ \mathrm{O} & -3.249 & 1.823 & 3.265 \\ \mathrm{O} & -2.161 & 2.648 & 5.058 \\ \mathrm{C} & -4.140 & 3.766 & 4.310 \\ \mathrm{H} & -5.088 & 3.280 & 4.571 \\ \mathrm{H} & -3.866 & 4.439 & 5.125 \\ \mathrm{C} & -4.353 & 4.531 & 2.993 \\ \mathrm{H} & -3.974 & 3.928 & 2.165 \\ \mathrm{H} & -3.777 & 5.456 & 2.967 \\ \mathrm{C} & -5.808 & 4.838 & 2.624 \\ \mathrm{O} & -6.727 & 4.032 & 2.957 \\ \mathrm{O} & -5.990 & 5.890 & 1.944\end{array}$

\begin{tabular}{lrrr}
\multicolumn{4}{c}{ Coordinate of } \\
3Pa-reb \\
$\mathrm{C}$ & 0.140 & 1.482 & 10.954 \\
$\mathrm{H}$ & 0.786 & 0.602 & 10.980 \\
$\mathrm{H}$ & -0.629 & 1.345 & 11.720 \\
$\mathrm{~N}$ & -1.613 & 2.332 & 9.302 \\
$\mathrm{H}$ & -2.227 & 2.771 & 9.996 \\
$\mathrm{C}$ & -0.449 & 1.638 & 9.588 \\
$\mathrm{C}$ & -1.748 & 2.382 & 7.953 \\
$\mathrm{H}$ & -2.570 & 2.859 & 7.444 \\
$\mathrm{~N}$ & -0.751 & 1.744 & 7.359 \\
$\mathrm{C}$ & 0.062 & 1.280 & 8.367 \\
$\mathrm{H}$ & 0.969 & 0.735 & 8.153 \\
$\mathrm{C}$ & 3.957 & 0.057 & 4.698 \\
$\mathrm{H}$ & 4.802 & 0.677 & 4.379 \\
$\mathrm{H}$ & 3.987 & -0.835 & 4.062 \\
$\mathrm{C}$ & 2.656 & 0.798 & 4.297 \\
$\mathrm{O}$ & 1.759 & 0.991 & 5.194 \\
$\mathrm{O}$ & 2.584 & 1.150 & 3.104 \\
$\mathrm{C}$ & 0.508 & 7.054 & 6.691 \\
$\mathrm{H}$ & -0.389 & 7.437 & 6.199 \\
$\mathrm{H}$ & 1.344 & 7.674 & 6.345 \\
$\mathrm{~N}$ & 1.952 & 4.972 & 6.428 \\
$\mathrm{H}$ & 2.834 & 5.339 & 6.799 \\
$\mathrm{C}$ & 0.729 & 5.622 & 6.314 \\
$\mathrm{C}$ & 1.806 & 3.714 & 5.977
\end{tabular}




\begin{tabular}{|c|c|c|c|}
\hline & 2.579 & 2.967 & 5.986 \\
\hline N & 0.564 & 3.507 & 5.554 \\
\hline c & -0.115 & 4.694 & 5.758 \\
\hline $\mathrm{H}$ & -1.147 & 4.792 & 5.465 \\
\hline $\mathrm{C}$ & -4.056 & -2.737 & 3.740 \\
\hline $\mathrm{O}$ & -4.938 & -2.252 & 4.445 \\
\hline $\mathrm{N}$ & -2.872 & -2.235 & 3.307 \\
\hline $\mathrm{C}$ & -2.069 & -1.064 & 3.527 \\
\hline $\mathrm{H}$ & -2.727 & -0.227 & 3.758 \\
\hline $\mathrm{C}$ & -1.100 & -1.312 & 4.682 \\
\hline $\mathrm{O}$ & -0.845 & -2.509 & 4.956 \\
\hline $\mathrm{O}$ & -0.578 & -0.312 & 5.261 \\
\hline $\mathrm{C}$ & -1.311 & -0.713 & 2.189 \\
\hline $\mathrm{C}$ & -2.260 & -1.028 & 1.002 \\
\hline $\mathrm{H}$ & -1.895 & 1.117 & 2.573 \\
\hline $\mathrm{H}$ & -3.204 & -0.492 & 1.136 \\
\hline $\mathrm{H}$ & -1.790 & -0.670 & 0.086 \\
\hline $\mathrm{C}$ & 0.055 & -1.382 & 2.009 \\
\hline $\mathrm{H}$ & 0.041 & -2.462 & 2.157 \\
\hline $\mathrm{H}$ & 0.418 & -1.191 & 0.996 \\
\hline $\mathrm{H}$ & 0.770 & -0.938 & 2.702 \\
\hline $\mathrm{S}$ & -2.621 & -2.825 & 0.700 \\
\hline $\mathrm{C}$ & -2.501 & -3.347 & 2.471 \\
\hline $\mathrm{H}$ & -1.521 & -3.771 & 2.691 \\
\hline $\mathrm{C}$ & -3.715 & -4.099 & 3.086 \\
\hline $\mathrm{H}$ & -3.347 & -4.762 & 3.873 \\
\hline $\mathrm{N}$ & -4.542 & -4.886 & 2.233 \\
\hline $\mathrm{H}$ & -4.986 & -4.429 & 1.446 \\
\hline $\mathrm{C}$ & -4.116 & -6.190 & 2.057 \\
\hline $\mathrm{O}$ & -3.385 & -6.717 & 2.899 \\
\hline $\mathrm{C}$ & -4.503 & -6.884 & 0.795 \\
\hline $\mathrm{H}$ & -5.501 & -6.595 & 0.460 \\
\hline $\mathrm{H}$ & -4.499 & -7.954 & 1.014 \\
\hline $\mathrm{C}$ & -3.507 & -6.560 & -0.342 \\
\hline $\mathrm{H}$ & -3.716 & -5.550 & -0.713 \\
\hline $\mathrm{H}$ & -2.483 & -6.561 & 0.049 \\
\hline $\mathrm{C}$ & -3.608 & -7.583 & -1.480 \\
\hline $\mathrm{H}$ & -3.146 & -7.169 & -2.386 \\
\hline $\mathrm{H}$ & -3.032 & -8.473 & -1.227 \\
\hline $\mathrm{N}$ & -5.871 & -6.867 & -2.140 \\
\hline $\mathrm{H}$ & -5.668 & -6.666 & -3.127 \\
\hline $\mathrm{H}$ & -6.829 & -7.218 & -2.149 \\
\hline $\mathrm{C}$ & -5.037 & -8.026 & -1.800 \\
\hline $\mathrm{H}$ & -5.466 & -8.489 & -0.901 \\
\hline $\mathrm{C}$ & -5.042 & -9.131 & -2.880 \\
\hline
\end{tabular}




$\begin{array}{lccc}\mathrm{O} & -4.088 & -9.959 & -2.822 \\ \mathrm{O} & -5.957 & -9.112 & -3.733 \\ \mathrm{Fe} & -0.186 & 1.609 & 5.261 \\ \mathrm{O} & -1.068 & 0.680 & 2.217 \\ \mathrm{C} & -3.002 & 2.617 & 4.005 \\ \mathrm{O} & -3.248 & 1.772 & 3.078 \\ \mathrm{O} & -1.984 & 2.602 & 4.751 \\ \mathrm{C} & -4.052 & 3.700 & 4.204 \\ \mathrm{H} & -4.990 & 3.190 & 4.451 \\ \mathrm{H} & -3.771 & 4.330 & 5.052 \\ \mathrm{C} & -4.303 & 4.543 & 2.940 \\ \mathrm{H} & -3.916 & 4.012 & 2.067 \\ \mathrm{H} & -3.756 & 5.486 & 2.968 \\ \mathrm{C} & -5.770 & 4.838 & 2.596 \\ \mathrm{O} & -6.678 & 4.020 & 2.928 \\ \mathrm{O} & -5.970 & 5.899 & 1.934\end{array}$

